University of Louisville

ThinkIR: The University of Louisville's Institutional Repository

$12-2018$

\title{
Association between cigarette smoking and ovarian reserve among women seeking fertility treatment with effect modification by race and NAT2 genotype.
}

Islamiat A. Oladipupo

University of Louisville

Follow this and additional works at: https://ir.library.louisville.edu/etd

Part of the Epidemiology Commons, and the Women's Health Commons

\section{Recommended Citation}

Oladipupo, Islamiat A., "Association between cigarette smoking and ovarian reserve among women seeking fertility treatment with effect modification by race and NAT2 genotype." (2018). Electronic Theses and Dissertations. Paper 3123.

https://doi.org/10.18297/etd/3123

This Doctoral Dissertation is brought to you for free and open access by ThinkIR: The University of Louisville's Institutional Repository. It has been accepted for inclusion in Electronic Theses and Dissertations by an authorized administrator of ThinkIR: The University of Louisville's Institutional Repository. This title appears here courtesy of the author, who has retained all other copyrights. For more information, please contact thinkir@louisville.edu. 
ASSOCIATION BETWEEN CIGARETTE SMOKING AND OVARIAN RESERVE

AMONG WOMEN SEEKING FERTILITY TREATMENT WITH EFFECT

MODIFICATION BY RACE AND NAT2 GENOTYPE

By

Islamiat A. Oladipupo

MPH, University of Louisville, 2014

MBBS, Olabisi Onabanjo University, 2006

\author{
A Dissertation \\ Submitted to the Faculty of the \\ School of Public Health and Information Sciences \\ of the University of Louisville \\ in Partial Fulfillment of the Requirements \\ for the Degree of
}

Doctor of Philosophy in Public Health Sciences

Department of Epidemiology and Population Health

University of Louisville

Louisville, Kentucky

December 2018 



\section{ASSOCIATION BETWEEN CIGARETTE SMOKING AND OVARIAN RESERVE AMONG WOMEN SEEKING FERTILITY TREATMENT WITH EFFECT MODIFICATION BY RACE AND NAT2 GENOTYPE}

By

Islamiat A. Oladipupo

MPH, University of Louisville, 2014

MBBS, Olabisi Onabanjo University, 2006

A Dissertation Approved on

November 9, 2018

by the following Dissertation Committee:

Dr. Kira Taylor, PhD, MS

Dr. Kathy Baumgartner, PhD

Dr. David W. Hein, PhD

Dr. Anne Wallis, PhD

Dr. Bakeerathan Gunaratnam, PhD 


\section{DEDICATION}

To my Late Dad, my number one Cheerleader, Alhaji Abdul-Hakeem Atunrase, I wish

you were here to celebrate this great accomplishment with me. You will always be in my heart. 


\section{ACKNOWLEDGEMENTS}

I would like to express my deepest gratitude to my mentor and dissertation committee chair, Dr. Kira Taylor. The opportunity you gave me to join your research team, your patient guidance, enthusiastic encouragement and useful critique of my dissertation is very much appreciated. I could not have imagined having a better advisor and mentor. I would like to thank my committee members: Dr. Kathy Baumgartner, Dr. David Hein, Dr. Anne Wallis and Dr. Bakeerathan Gunaratnam. I thank you for your time, professional guidance and valuable support. I would also like to thank the Louisville Tobacco Smoke Exposure, Genetic Susceptibility and Infertility Study (LOUSSI) student research staff: Emily Steinmetz, T'shura Ali and Sashia Torres; Mark Doll for helping with the NAT2 genotyping and analysis.

My profound gratitude to Prof. Richard Baumgartner for your never-ending support right from my MPH days at the department of Epidemiology. Thank you to my mum, Alhaja Risikat Atunrase and my sister, Basirat Bashua for their unflinching support and constant words of encouragement. To my husband, Abolaji Oladipupo, I could not have achieved this without your support, love, patience and understanding. Your belief in me kept me going. To my children, Haleema and Farooq, thank you for your unconditional love.

Finally, I would like to acknowledge the co-investigators of the LOUSSI study, Fellows and the staff of the University of Louisville Fertility Clinic and the funding source that 
made this research possible: National Institutes of Health (NIH), Eunice Kennedy Shriver National Institute of Child Health and Human Development (NICHD), "The Interaction between NAT2 Acetylator Status and Exposure to Tobacco Smoke on Ovarian Reserve and In Vitro Fertilization Outcomes" (Grant: 1R15HD087911-01 Principal Investigator (PI): Kira Taylor). 


\begin{abstract}
ASSOCIATION BETWEEN CIGARETTE SMOKING AND OVARIAN RESERVE AMONG WOMEN SEEKING FERTILITY TREATMENT WITH EFFECT MODIFICATION BY RACE AND NAT2 GENOTYPE

Islamiat A. Oladipupo

November 9, 2018

Cigarette smoking in women has been associated with adverse reproductive outcomes such as reduced ovarian reserve, poorer in vitro fertilization (IVF) outcomes and increased adverse pregnancy outcomes. This study examined the association of smoking with ovarian reserve in a cross-sectional study of women seeking fertility treatment, and potential effect modification by race and NAT2 acetylator phenotype.

Data from 265 women from the Louisville Tobacco Smoke, Genetic Susceptibility, and Infertility (LOUSSI) Study were analyzed. A total of 265 women were recruited through a single infertility clinic between September 2016 and June 2018. Information on current smoking status was assessed using a structured questionnaire and confirmed by cotinine assay. Single nucleotide polymorphisms in NAT2 were genotyped to determine acetylator status and serum anti-Müllerian hormone (AMH) level was used to assess ovarian reserve. The association of smoking with ovarian reserve was assessed using linear and logistic regression models with adjustment for potential confounders.
\end{abstract}


Effect modification by race and NAT2 phenotype were assessed by including interaction terms in the regression models.

Overall, smoking was not significantly associated with ovarian reserve. Results suggest that heavy smoking and higher pack-years of exposure may decrease ovarian reserve. Although most associations were not statistically significant, the effect of smoking on ovarian reserve was more pronounced among non-Hispanic Black women and slow NAT2 acetylators. These results are based on a small clinical population and require replication in a larger and more representative study population. 
PAGE

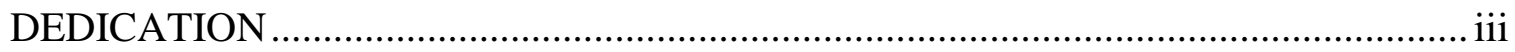

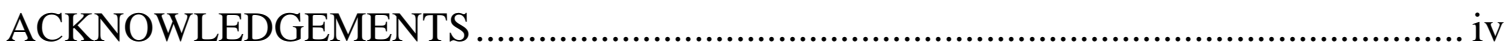

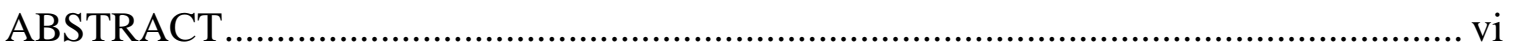

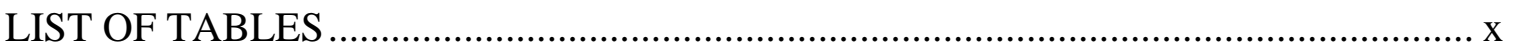

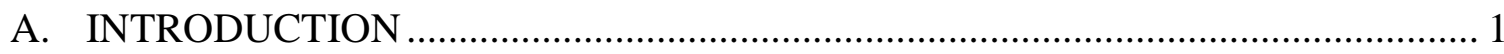

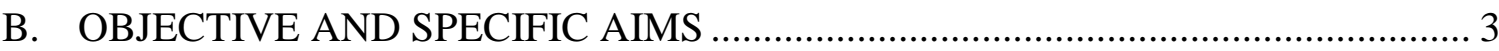

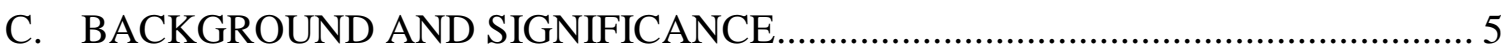

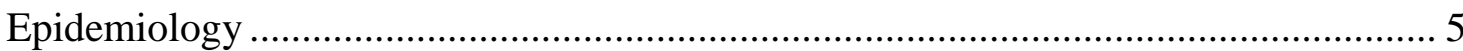

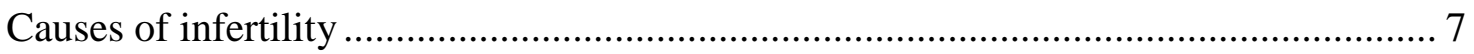

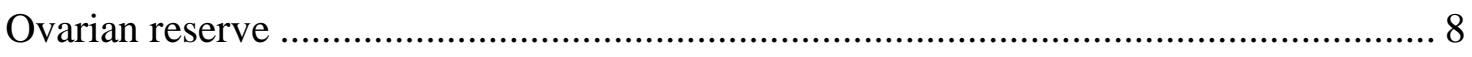

Antimullerian Hormone (AMH) ..................................................................... 9

Antral Follicle Count (AFC)............................................................................ 10

Predictors/ Determinants of Ovarian reserve............................................................. 11

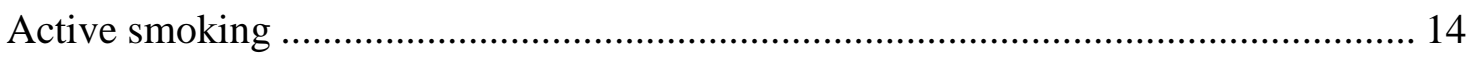

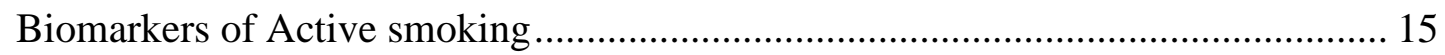

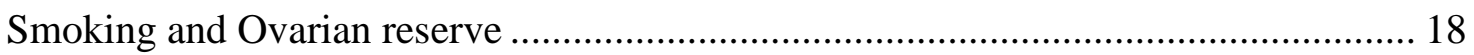

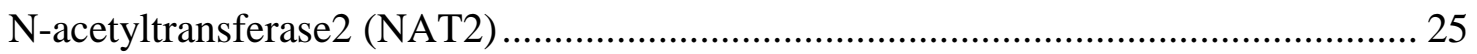

Summary and Gap in Current Knowledge ............................................................. 28

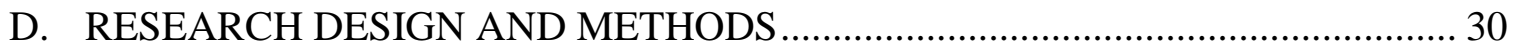

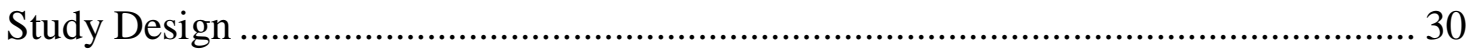

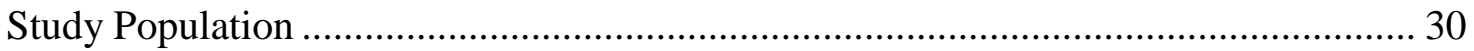

Subject Recruitment Methods ……………………......................................... 32

Exposure Assessment ..................................................................................... 32

Outcome Measurement ………………......................................................... 33

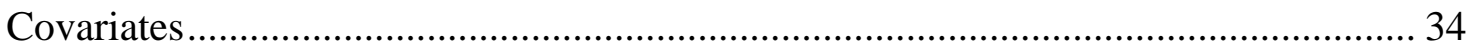

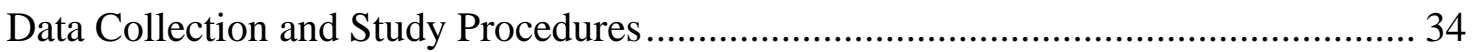

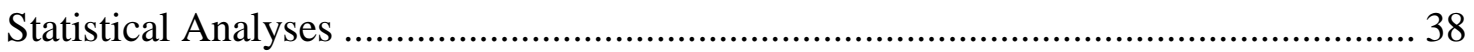


Sample Size / Power calculation ...................................................................... 42

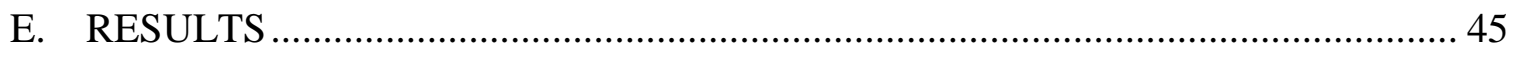

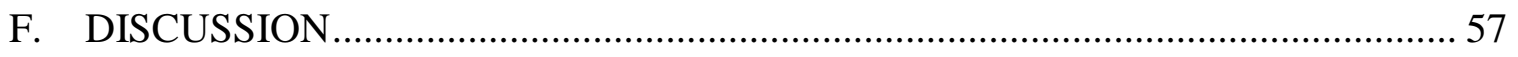

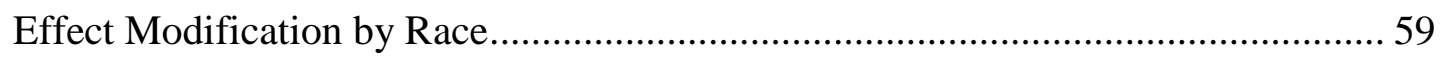

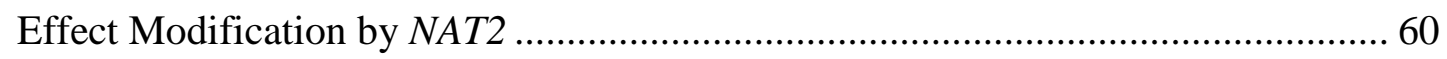

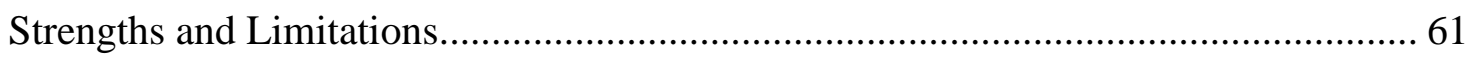

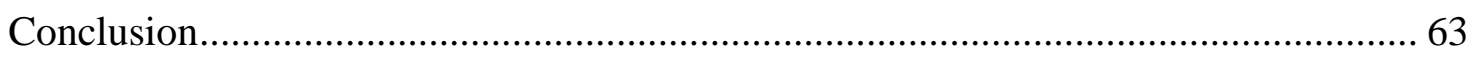

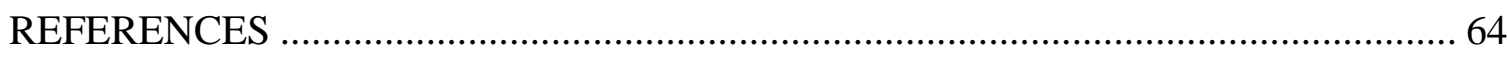

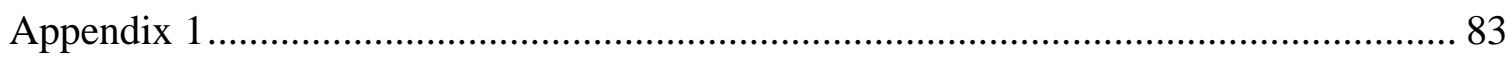

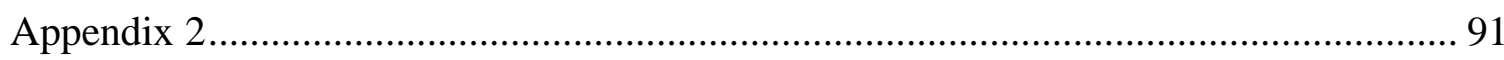

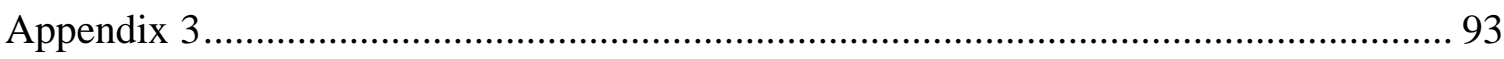

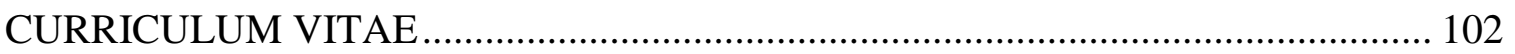




\section{LIST OF TABLES}

Table 1: Varying urinary-cotinine cut-points ......................................................... 16

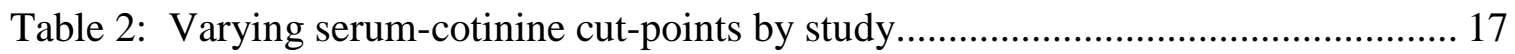

Table 3: Summary of Studies on Active Smoking and AMH Levels ........................... 22

Table 3: Summary of Studies on Active Smoking and AMH Levels Continuation ......... 23

Table 3: Summary of Studies on Active Smoking and AMH Levels Continuation ......... 24

Table 4. 1: Sample size estimate for the difference in mean AMH levels between smokers

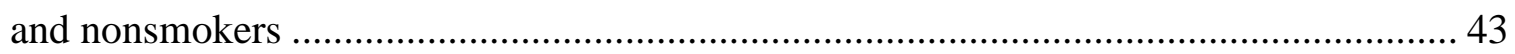

Table 4. 2: Sample size estimate for different odds ratio for effect of smoking on DOR 43

Table 4. 3: Power calculations for detecting a significant gene-environment interaction

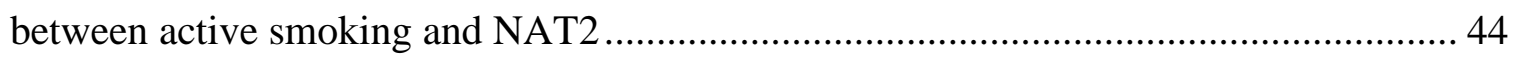

Table 4. 4: Sample size estimate stratified by NAT2 acetylator status ......................... 44

Table 5. 1. Characteristics of Women in the LOUSSI Study Stratified by Smoking Status

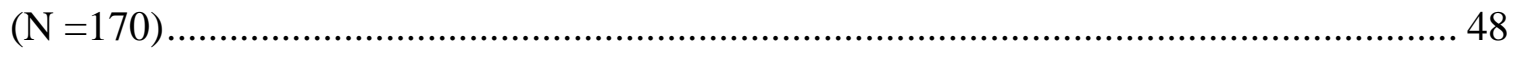

Table 5. 2. NAT2 Phenotype of Women in the LOUSSI Study $(\mathrm{N}=156)$....................... 49

Table 5. 3. Univariate Analyses for the Association of Current Active Smoking with

AMH and Diminished Ovarian Reserve (DOR) .................................................. 51

Table 5. 4. Multivariable Model for Association of Current Active Smoking with AMH

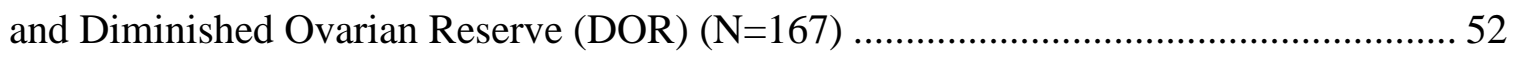


Table 5. 5. Association of Current Active Smoking with DOR Among Women Without

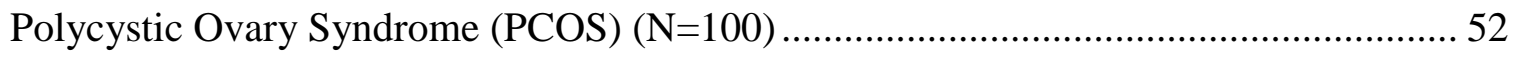

Table 5. 6. Association of Cumulative Lifetime Smoking with AMH and DOR(N=204)53

Table 5. 7. Association of Cumulative Lifetime Smoking with DOR Among Women

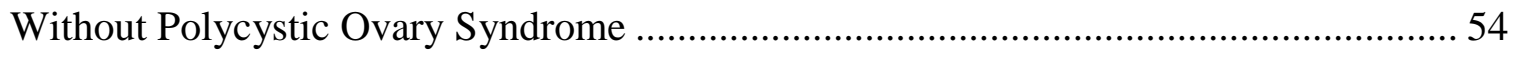

Table 5. 8. Interaction of Current Active Smoking and Race on Diminished Ovarian

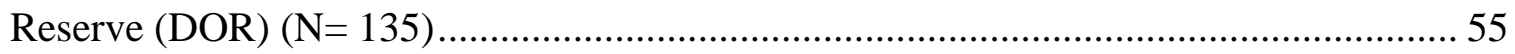

Table 5. 9. Interaction of Current Active Smoking and Race on Diminished Ovarian

Reserve (DOR) Among Women without Polycystic Ovary Syndrome (N=82)............. 55

Table 5. 10. Interaction of Current Active Smoking and NAT2 Acetylator Phenotype on

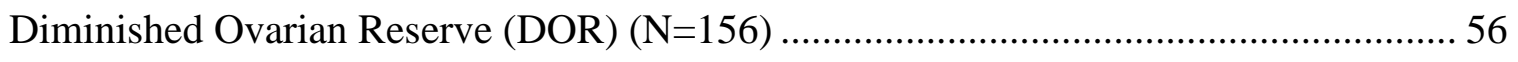

Table 5. 11. Interaction of Current Active Smoking and NAT2 Acetylator Phenotype on Diminished Ovarian Reserve (DOR) Among Women Without Polycystic Ovary

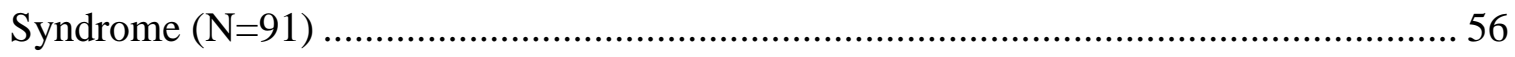




\section{A. INTRODUCTION}

Infertility and impaired fecundity among women of reproductive age remain an important public health issue. It is estimated that $10.9 \%$ of women between the ages of 15-44 have impaired fecundity [1]. Among married women 15-44 years of age, $6.0 \%$ are infertile and non-Hispanic black women are more likely to be infertile than non-Hispanic white (NHW) women [1]. Among women aged 15-44, 12\% had ever used infertility services with use highest among older women, NHW women and women with higher educational levels and income $[1,2]$. The median per-person costs of infertility treatment ranged from $\$ 1,182$ for medications only, to $\$ 24,373$ and $\$ 38,015$ for in-vitro fertilization (IVF) and IVF with donor egg groups, respectively. Eighty- five percent of IVF costs are paid out of pocket $[3,4]$. In addition to the financial burden, the diagnosis and treatment of infertility is associated with psychological and emotional consequences such as anxiety and depression $[5,6]$.

Causes of infertility include ovulatory disorders, tubal damage, uterine or peritoneal problems and male factors, and in $15 \%$ to $30 \%$ of cases, etiology is unknown $[7,8]$. Ovulatory dysfunction or ovarian failure accounts for $20 \%$ of cases of infertility [8]. Diminished ovarian reserve (DOR) defined as a condition where the response to ovarian stimulation or fecundity is reduced compared with women of comparable age, 
is present in about $27.5 \%$ of women undergoing IVF [9]. Markers of ovarian reserve include anti-Müllerian hormone (AMH) serum levels and antral follicle count (AFC) as assessed by transvaginal ultrasound $[10,11]$. AMH levels and AFC are important predictors of IVF success with lower levels reflecting smaller follicular pool and ovarian reserve [10-15]. Major predictors of ovarian reserve include age, age at menarche, parity, obesity and race/ethnicity [16].

Cigarette smoking in women has been associated with adverse reproductive outcomes such as reduced ovarian reserve, poorer IVF outcome and increased adverse pregnancy outcomes [17-22]. Studies of the association of active smoking with ovarian reserve have been inconsistent. No epidemiological studies have been conducted to explore potential gene-environment interaction as a possible cause of inconsistency in results $[23,24]$.

$\mathrm{N}$-acetyltransferase2 (NAT2) is an important enzyme in the conjugation of certain drugs and other xenobiotics such as tobacco smoke, caffeine and pesticides $[25,26]$. The effects of NAT2 polymorphisms and their interaction with smoking on different cancer risks have been established [27-29]. However, only one epidemiological study has been conducted on the effects of NAT2 polymorphisms and possible interaction with smoking on fertility and related outcomes among women [24]. No studies have explored the potential interaction between smoking and NAT2 phenotypes on ovarian reserve. 


\section{B. OBJECTIVE AND SPECIFIC AIMS}

The primary objective of the study is to examine the relationship between active cigarette smoking and ovarian reserve as measured by AMH levels in females seeking fertility treatment. Racial disparity by ethnicity (African-American, NHW) will be explored by comparing the association in African-American (AA) with NHW women. In addition, potential effect modification by NAT2 acetylator status on ovarian reserve will be evaluated.

The specific aims of the study are as follows;

1(a). To determine if current active smoking, as measured by urinary cotinine levels and questionnaire, is associated with ovarian reserve (as measured by $\mathrm{AMH}$ ) among women seeking fertility treatment after controlling for potential confounders such as age, body mass index (BMI), age at menarche, parity and PCOS status.

Hypothesis: Smoking is associated with ovarian reserve with smokers having decreased ovarian reserve compared to nonsmokers.

1(b). To determine if a dose-response relationship exists between current active smoking (as measured by number of cigarettes smoked per day) and ovarian reserve. The dose of exposure will be assessed based on response to the smoking questionnaire. 
Hypothesis: A dose-response relationship exists between active smoking and ovarian reserve with a higher level of exposure being associated with decreased ovarian reserve.

1(c). To determine if a dose-response relationship exists between cumulative lifetime smoking (as measured by pack-years reported on the questionnaire) and ovarian reserve.

Hypothesis: A dose-response relationship exists between cumulative lifetime exposure to smoking and ovarian reserve with longer pack-years of exposure being associated with decreased ovarian reserve.

2. To determine if the association of current active smoking with ovarian reserve differ by race, comparing African-American with Caucasians (NHW) women. Hypothesis: The associations between active smoking and ovarian reserve will be different when comparing AA to NHW women (i.e. there is a significant interaction between smoking and race when examining their joint effects on ovarian reserve).

3. To determine if there is an interaction between current active smoking and NAT2 acetylator status on ovarian reserve.

Hypothesis: There is a significant interaction between smoking and NAT2 acetylator status when examining their joint effects on ovarian reserve, with slow acetylators having lower ovarian reserve compared to intermediate and rapid acetylators. 


\section{BACKGROUND AND SIGNIFICANCE}

Infertility is defined as failure to achieve clinical pregnancy after 12 months or more of regular unprotected sexual intercourse [30]. A diagnosis of infertility is made based on detailed medical history and physical examination followed by diagnostic evaluations to identify the underlying etiology [11]. Diagnostic evaluation for infertility includes tests for ovarian function, uterine abnormalities, cervical factors, tubal patency, peritoneal factors and semen analysis [11,31,32].

Infertility is divided into two major subtypes of primary and secondary infertility [30]. Secondary infertility refers to the inability of a woman to get pregnant or carry a pregnancy to live birth following a previous pregnancy or live birth. Diagnosis of primary infertility is made in women with no prior history of conception or live birth [30].

\section{Epidemiology}

Globally, infertility affects 48.5 million couples. Of these, 19.2 million have primary infertility and 29.3 million have secondary infertility [33]. The prevalence of infertility has increased globally [33]. In 2010, the prevalence of secondary infertility increased with age, from $2.6 \%$ in women aged $20-24$ to $27.1 \%$ in women aged $40-44$ years [33]. The trends were reversed for primary infertility with prevalence higher among 
women aged 20-24 (2.7\%) compared to women aged 25-29 (2.0\%) and women aged 30$44(1.6 \%)[33]$.

In the United States, the percentage of all married women 15-44 years of age who are infertile was $6.0 \%$ between 2006 to 2010 and increased to $6.7 \%$ in 2015 [1,34]. The prevalence of infertility increased with age. Among currently married women 15-44 years with no prior live birth, the prevalence was $8.7 \%$ among women aged $15-29,11.0 \%$ in women $30-34,23.0 \%$ in women $35-39$ and $26.2 \%$ in women $40-44$ years [34].

An estimated 7.3 million women $15-44$ years have ever received any infertility services between 2011 to 2015 [2, 34]. The use of infertility services varied by socioeconomic status and race/ethnicity. Among women 25-44 years of age, ever use of fertility services ranged from $9.5 \%$ in women with no high school diploma to $22.6 \%$ in women with a master's degree or higher [2]. Use of fertility service was higher among women $300-399 \%$ the above poverty level (21.2\%) compared to women less than $100 \%$ below poverty level (12.9\%) [2]. Among the racial/ethnic groups, Non-Hispanic black (African-Americans) had lower ever use of fertility services $(11.0 \%)$ than non-Hispanic white $(19.1 \%)$, or Hispanic (13.2\%) women [2].

Some of the disparities in use of infertility services have been linked to the significant costs of medical services and the lack of adequate health insurance to cover necessary diagnostic tests or treatments $[2,35,36]$. The estimated median cost per IVF cycle rose from $\$ 9226$ in 2001 to about $\$ 12,513$ in $2006[37,38]$. The median per-person cost of infertility treatment ranged from $\$ 1,182$ for medications only, to $\$ 24,373$ and $\$ 38,015$ for invitro fertilization (IVF) and IVF with donor egg groups, respectively [3]. In 
the United States, only $25 \%$ of health care plans cover infertility treatment with $85 \%$ of IVF costs being paid out of pocket $[4,36]$.

Aside from the financial burden associated with infertility, the condition is associated with psychological and emotional consequences such as anxiety and depression $[5,6]$. In a cross-sectional survey of infertile women referred for infertility treatment, $40.8 \%$ had depression and $86.8 \%$ had anxiety [5]. The duration of infertility predicted $18 \%$ of anxiety and $25 \%$ of depression among infertile women [6].

\section{Causes of infertility}

Conception requires the timely release of a matured oocyte; adequate number of progressively motile and normal spermatozoa capable of reaching and fertilizing the oocyte; patent fallopian tubes for free passage of the sperm to reach the oocyte for fertilization and migration of zygote/embryo to the uterus; and a well primed endometrium that allows for implantation [11, 31, 32]. Biological, anatomical or functional defects at any of these stages may lead to infertility. The probable causes of infertility are classified into tubal factor, uterine or peritoneal problems, male factor and ovulatory dysfunction $[7,8]$. An estimated $40 \%$ of couples with infertility have a combination of factors while $15 \%$ may not display any objective alterations in fertility function [8].

Tubal factors account for $14 \%$ of cases of infertility and include conditions that causes tubal obstruction and /or peritoneal adhesions [7]. Conditions that affect the integrity of the uterine cavity and peritoneum such as endometriosis, uterine fibroids, uterine synechiae may impact fertility $[7,8,11,32]$. The male factors include conditions 
that affects the concentration, motility, vitality and morphology of sperm cells $[7,8,31$, 32].

Ovulatory Dysfunction. Ovulation normally occurs when an ovary releases a single mature oocyte every month and women who ovulate typically have regular and consistent menstrual duration and flow [7]. Ovulatory dysfunction commonly results in menstrual disturbances and account for $40 \%$ of infertility in women [11, 39]. Causes of ovulatory dysfunction include polycystic ovary syndrome (PCOS), obesity, weight gain or loss, strenuous exercise, thyroid dysfunction, and hyperprolactinemia [7, 8, 11]. Methods of assessing ovulatory dysfunction include detailed menstrual history, physical and gynecological examination, basal temperature measurements, ultrasound, serum progesterone level and endometrial biopsy [11].

\section{Ovarian reserve}

Ovarian reserve describes reproductive potential as a function of the number and quality of oocytes [10]. It reflects the number of oocytes remaining in the ovaries that influences the probability of getting pregnant [7]. Decreased or diminished ovarian reserve (DOR) describes women of reproductive age having regular menses whose response to ovarian stimulation or fecundity is reduced compared with those women of comparable age [7].

Diagnostic criteria for DOR is poorly defined as standardized definition is lacking $[10,40]$. Definition and diagnostics criteria of DOR varies across fertility clinics and studies [40]. Evidence suggest an upward trend in the prevalence of DOR among assisted reproductive technology (ART) patients $[41,42]$. Devin et al. reported an increase in the prevalence of DOR from 19 to $26 \%$ from 2004 to 2011 in a retrospective study of 
181,536 ART cycles reported to Society for Assisted Reproductive Technologies (SART) by United States clinics [41]. In a cross-sectional study using ART cycles between 20042007, a significant secular trend of increased odds of DOR diagnosis was observed [42]. The adjusted odds ratio of DOR per year studied was 1.11 (95\% CI: 1.09-1.13), which translates to a 23\% higher odd of DOR in 2005 compared to 2004, 37\% higher odds of DOR in 2006 compared to 2004, and 52\% higher odds of DOR in 2007 compared to $2004[42]$.

Measures of the ovarian reserve have been used to predict DOR and counsel infertile couples on choice of treatment $[7,10]$. Tests of ovarian reserve include early follicular phase antral follicle count (AFC) using transvaginal ultrasonography and biochemical tests $[7,10,11]$. The biochemical tests include basal measurements of follicle-stimulating hormone (FSH), estradiol, inhibin $\mathrm{B}$, and antimullerian hormone (AMH), and the clomiphene citrate challenge test (CCCT) [7, 10, 11]. Antral follicle count and serum AMH have good predictive value and are the preferred methods for assessing ovarian reserve [43-45].

\section{Antimullerian Hormone (AMH)}

Anti-Müllerian hormone (AMH), also called Müllerian inhibiting substance (MIS), is a glycoprotein hormone belonging to the large family of transforming growth factors- $\beta$ (TGF- $\beta$ ) [10]. In women, AMH is expressed uniquely by the ovary in the granulosa cells, primarily secreted by primary, preantral and antral follicles and are direct measures of the follicular pool $[10,11]$. As the number of ovarian follicles declines with age, AMH concentrations decline [16]. Serum concentrations of AMH are gonadotropinindependent and therefore remain relatively constant within and between menstrual 
cycles in both normal, young, ovulating women and in women with infertility, making it a valuable and reliable marker of ovarian function [10, 14, 46-48].

The following are considered the lower limit of age-appropriate serum AMH values for these 5-year age intervals: $0.5 \mathrm{ng} / \mathrm{ML}$ for 45 years, $1 \mathrm{ng} / \mathrm{mL}$ for 40 years, $1.5 \mathrm{ng} / \mathrm{mL}$ for 35 years, $2.5 \mathrm{ng} / \mathrm{mL}$ for 30 years and $3.0 \mathrm{ng} / \mathrm{mL}$ for 25 years [44]. Thus, $\mathrm{AMH}$ level $\geq 1.0 \mathrm{ng} / \mathrm{mL}$ but $\leq 3.5 \mathrm{ng} / \mathrm{mL}$ if age appropriate is consistent with normal ovarian response to ovarian stimulation. Lower serum AMH levels $(<1 \mathrm{ng} / \mathrm{mL})$ have been associated with poor responses to ovarian stimulation, poor embryo quality and poor pregnancy outcomes in IVF patients [13, 15, 49-51].

Varying levels of serum AMH have been used to predict ovarian response to stimulation. Serum cut-points ranging from 0.2 to $0.7 \mathrm{ng} / \mathrm{ml}$ have been associated with poor ovarian response with sensitivity and specificity ranging from 40-97\% and 78-92\%, respectively [10]. In a retrospective study of one hundred and eight IVF patients, AMH with a cutoff of $0.2 \mathrm{ng} / \mathrm{mL}$ predicted poor response to ovarian stimulation with a sensitivity of $87 \%$ and specificity of $64 \%$ [51]. In a prospective study of one hundred thirty-five women undergoing the first cycle of assisted reproduction treatment (ART), serum AMH predicted poor ovarian response with a sensitivity of $100 \%$ and specificity of $73 \%$ at an optimum cutoff value of $\leq 0.99 \mathrm{ng} / \mathrm{mL}$ [45]. In a meta-analysis that included 28 studies, AMH was found to have good predictive value for poor ovarian response with an area under the curve (AUC) of 0.78 [52].

\section{Antral Follicle Count (AFC)}

Antral follicle count (AFC) is the sum of antral follicles in both ovaries, as observed with transvaginal ultrasonography during early follicular phase (day 2-4) of the 
menstrual cycle $[11,44]$. Antral follicles are defined as those measuring 2-10mm in mean diameter in the greatest two-dimensional plane $[11,44]$. The AFC correlates with histologically determined number of primordial follicles and capacity of the ovary to produce oocytes in either an assisted reproduction setting or through natural folliculogenesis [53]. In experienced centers, AFC has good inter-cycle reliability and inter-observer reliability $[11,44]$.

Low AFC, considered to be less than or equal 6 total antral follicles, is associated with poor response to ovarian stimulation during IVF $[11,44,54]$. AFC $\leq 10$ predicted poor ovarian response with sensitivity of $93 \%$ and specificity of $88 \%$ with an AUC of 0.935 in prospective study of one hundred thirty-five women undergoing the first cycle ART [45]. AUC for AFC in predicting poor ovarian response was found to be 0.76 in a meta-analysis of 28 studies on IVF [52].

Studies have shown that AFC is highly correlated with AMH level $[55,56]$. Antral follicles measuring $<6 \mathrm{~mm}$ express the greatest amount of $\mathrm{AMH}$, and levels decline as antral follicles increase in size [57]. Both AFC and AMH have strong and similar linear relationship with the size of the primordial follicle pool and ovarian reserve [43-45, 58]. The limited intra- and inter-cycle variation, objectivity and potential standardization of AMH assays, makes it the preferred biomarker of ovarian reserve in women.

\section{Predictors/ Determinants of Ovarian reserve}

Current age and Age at menarche. Older women and women with earlier age at menarche have been shown to have lower levels of AMH reflecting a decline in the ovarian pool $[12,16]$. In a cross-sectional study of premenopausal women conducted by Jung et al., women 40 years and older had significantly lower AMH concentrations 
$(0.73 \mathrm{ng} / \mathrm{ml})$ compared to women less than 35 years of age $(2.52 \mathrm{ng} / \mathrm{ml})$ [12]. AMH concentrations were also significantly lower among women with age at menarche less than 12 years $(0.90 \mathrm{ng} / \mathrm{mL})$ compared to women with age at menarche $\geq 14$ years $(1.12$ $\mathrm{ng} / \mathrm{mL})[12]$.

Race/ethnicity. The association of race/ethnicity with AMH levels is inconsistent. In the multi-ethnic cohort study of ovarian aging (OVA), mean AMH levels were significantly lower in AA women $(22.8 \pm 1.7 \mathrm{pM})$ compared to Caucasians $(30.1 \pm$ $1.5 \mathrm{pM})$ [16]. The rate of decrease in level of AMH also varied by race. In Caucasian women, AMH decreased by $2 \mathrm{pM}$ per year, while in African American women it decreased by $0.84 \mathrm{pM}$ per year [16]. Studies that reported no association between race and AMH levels were cross-sectional and majorly of single ethnic group (Asian and Caucasian) with under-representation of other races [12, 59, 60].

Parity. High parity has been postulated to have a protective effect on ovarian reserve by reducing follicular recruitment [61]. In cross-sectional study of 2320 women aged 20 to 59 years, high parity was found to be associated with higher age-specific AMH levels $(\mathrm{p}=0.02)$ [59]. Not all studies found a positive association between parity and AMH levels. Bragg et al. found a statistically significant inverse association between parity and AMH: women with $2(\mathrm{p}<0.005)$ or 3 or more $(\mathrm{p}<0.01)$ children had lower AMH levels than nulliparous women [62]. Bleil et al. also found having had one or more live births versus being nulliparous was associated with a $31.1 \%$ (95\% CI: $21.6 \%, 39.5 \%)$ reduction in $\mathrm{AMH}[61]$.

Oral contraceptive pill (OCP) use. Current use of OCP has been linked with lower levels of AMH $[12,59]$. Concentrations of AMH were significantly lower among 
current users of oral contraceptives $(0.36 \mathrm{ng} / \mathrm{ml})$ compared with never or former users (1.15 ng/mL) [12].

Polycystic Ovarian Syndrome (PCOS). Multiple epidemiological investigations have consistently demonstrated that women with diagnosis of PCOS have significantly elevated serum AMH levels. In a hospital-based case-control study, mean serum AMH level was markedly increased in the PCOS group (47.1 +/- 22.9 compared to. $20.8+/-$ $11.6 \mathrm{pmol} / \mathrm{liter}$ in controls; $\mathrm{p}<0.0001$ ) [63]. In a retrospective study of women evaluated for infertility, 97\% of women with AMH >10ng/ml had PCOS [64].

Past Ovarian Surgery. Ovarian surgery is associated with decreased ovarian reserve possibly due to decrease in ovarian volume $[65,66]$. In women with regular cycle, serum AMH was undetectable after bilateral oophorectomy [67]. Similarly, serum AMH levels were significantly decreased after 1 -week post-cystectomy ( median $=0.67$; range $=0.02-1.93 ; 95 \% \mathrm{CI}: 0.44-1.70 \mathrm{ng} / \mathrm{mL}$ ), and subsequently increased to about $65 \%$ of the preoperative level after 3 months $(1.50$; range $=0.58-3.27 ; 95 \%$ CI: $0.58-3.26$ $\mathrm{ng} / \mathrm{mL})[65]$.

Obesity. The effect of obesity, body mass index (BMI) of at least $30 \mathrm{~kg} / \mathrm{m}^{2}$, on ovarian reserve is inconsistent among studies. In a population-based cohort of one hundred and twenty-two late reproductive-age (35-47 years) women, a significant inverse association of AMH levels with BMI was observed [68]. Obese women had mean AMH levels that were $65 \%$ lower than non-obese women (geometric mean ratio $(\mathrm{GMR})=0.35$; 95\% CI: $0.13,0.92 ; p=0.034)$ [68]. Among ovulatory women ages 18 to 35 years, AMH levels were $34 \%$ lower in the obese group $(2.9 \pm 2.1 \mathrm{ng} / \mathrm{mL})$ compared to normal group (4.4 $\pm 1.8 \mathrm{ng} / \mathrm{ml}), \mathrm{p}<0.05$ [69]. Buyuk et al. found a similar inverse association between 
BMI and AMH but only among women with DOR [70]. Among women with DOR, overweight and obese women had 33\% lower AMH levels than those with a normal BMI $(0.4 \pm 0.3$ vs. $0.6 \pm 0.5$, respectively; $\mathrm{p}=0.0001)$; no association was found between BMI and $\mathrm{AMH}$ among women with normal ovarian reserve [70]. The negative association between BMI and AMH was not replicated in other studies [59, 71-73].

\section{Active smoking}

Exposure to tobacco smoke has been associated with harmful reproduction ranging from decreased ovarian reserve and infertility to poor pregnancy outcome [1719]. Some of the constituents of tobacco smoke known to have toxic effects on reproductive health include, carbon monoxide, nicotine, cadmium and polycyclic aromatic hydrocarbons (PAHs) such as benzo[a]pyrene (B[a]P) [74, 75].

Most of the evidence on probable biological mechanism on how exposure to tobacco smoke may influence ovarian reserve and reproduction is based on experimental studies in animals. Some of the proposed biological mechanism through which tobacco smoke influences ovarian reserve includes inhibition of follicular development; premature luteinization of the preovulatory follicle; reduction of oocyte vascularization and maturation; atresia of oocytes in primordial and small primary follicles; impaired steroidogenesis; increased chromosomal errors; and cytotoxicity [75, 76]. Benzo[a]pyrene $(\mathrm{B}[\mathrm{a}] \mathrm{P})$, present in cigarette smoke, has been shown to significantly affect the phase I enzymes and cell death genes during preantral/antral and preovulatory growth leading to apoptosis and decreased viability [77]. Benzo[a]pyrene exposure decreased AMH output overall during preantral and antral follicle development further confirming the adverse effects of cigarette smoking on follicular development and survival [78]. 
Cadmium, found in tobacco smoke, accumulates in ovarian follicles and inhibits the expression of P450scc leading to impaired ovarian steroidogenesis [79-81]. Cadmium was also shown to increase ovarian oxidative stress [82]. Increased lipid peroxidation, reduced glutathione contents, increased catalase activity and decreased superoxide dismutase activity were observed in ovaries exposed to cadmium [82].

Smokers have been shown to secrete significantly higher amounts of vascular endothelial growth factor (VEGF) soluble receptor 1, which may result in decreased availability of VEGF and impaired angiogenesis and oocyte maturation [83].

Based on data from the National Health Interview Survey (NHIS), 13.6\% of U.S. women 18 years and above were a current cigarette smoker in 2015 [84]. The prevalence of cigarette smoking was higher among non-Hispanic white (16.0\%) followed by African -Americans (13.3\%) and Hispanics (7.1\%) [84]. In Kentucky, 24\% of women 18 years and older were a current smoker in 2016 [85].

\section{Biomarkers of Active smoking}

Cotinine is a major proximate metabolite of nicotine and reflects exposure to other constituents of tobacco smoke. It is measured in plasma, saliva and urine with a half-life ranging from 7-40 hours in adults, reflecting up to 3-5 days of exposure to tobacco smoke [86-88]. Urinary cotinine is a widely used biomarker of exposure to tobacco smoke. The advantages of urinary cotinine as biomarker of tobacco smoke exposure are that cotinine concentrations and other metabolites are higher than in other biological fluids; it represents relatively acute exposure; and collection is non-invasive [87]. The urinary cut-off of greater than or equal to $2.47 \mathrm{ng} / \mathrm{ml}$ have been shown to detect active smoking with the highest 
sensitivity and specificity of $100 \%$ [89]. Table 1 gives a breakdown of various cut-off values of urinary cotinine for smoking using receiver operating curve (ROC) [89].

Table 1: Varying urinary-cotinine cut-points

\begin{tabular}{|l|l|l|}
\hline Urinary cotinine $(\mathrm{ng} / \mathrm{ml})$ & Sensitivity & 1 -specificity \\
\hline$\geq 2.47$ & 1.000 & 1.000 \\
\hline$\geq 12.54$ & 0.909 & 0.902 \\
\hline$\geq 20.91$ & 0.682 & 0.843 \\
\hline$\geq 50.59$ & 0.500 & 0.588 \\
\hline$\geq 94.34$ & 0.455 & 0.549 \\
\hline$\geq 105.56$ & 0.455 & 0.539 \\
\hline
\end{tabular}

Levels of cotinine in urine and blood are highly correlated, with urine to blood ratio of 5 (correlation coefficient $=0.78,95 \% \mathrm{CI}$ : 0.61 to 0.88 ) [90]. Levels of serum cotinine used to distinguish between active cigarette smoking and exposure to secondhand smoke (SHS) vary by study (table 2). Levels greater than $10 \mathrm{ng} / \mathrm{ml}$ have been associated with recent active smoking while levels between $0.05-10 \mathrm{ng} / \mathrm{ml}$ indicates a SHS exposure in a non-smoker [91]. Another example of cut-points used to differentiate between active smoking and exposure to SHS and nonsmoking are cotinine levels of $\geq 14 \mathrm{ng} / \mathrm{mL}, 0.5-13.9 \mathrm{ng} / \mathrm{mL}$, and $<0.5 \mathrm{ng} / \mathrm{ml}$, respectively [88]. An optimal cutpoint determined using NHANES data (1999-2004) for distinguishing smokers from nonsmoker was $3 \mathrm{ng} / \mathrm{ml}$ [92]. Recommended evaluation of cut- points by the authors differ by race/ethnicity: 6ng/ml, for African-American, $5 \mathrm{ng} / \mathrm{ml}$ for non-Hispanic whites and 1ng/ml for Mexican American [92]. The variations in cut-points are due to differences in smoking behavior and slower metabolism or clearance of cotinine in blacks [86, 92]. 
Table 2: Varying serum-cotinine cut-points by study

\begin{tabular}{|c|c|c|c|c|}
\hline Author & $\begin{array}{l}\text { Study } \\
\text { design }\end{array}$ & Study population & $\begin{array}{l}\text { Study } \\
\text { objective }\end{array}$ & Cut-points \\
\hline $\begin{array}{l}\text { Homa et } \\
\text { al. (2015) }\end{array}$ & $\begin{array}{l}\text { Cross- } \\
\text { sectional }\end{array}$ & $\begin{array}{l}\text { U.S. population } \\
\text { (NHANES)- } \\
\text { Aged } 3 \text { years and } \\
\text { older } \\
\text { Both sexes } \\
\text { All races }\end{array}$ & $\begin{array}{l}\text { Examine } \\
\text { SHS } \\
\text { exposure } \\
\text { among } \\
\text { nonsmoking } \\
\text { population }\end{array}$ & $\begin{array}{l}\text { SHS exposure }=0.05- \\
10 \mathrm{ng} / \mathrm{mL} \\
\text { Active smoking }= \\
>10 \mathrm{ng} / \mathrm{ml}\end{array}$ \\
\hline $\begin{array}{l}\text { Benowitz } \\
\text { et al. } \\
(2009)\end{array}$ & $\begin{array}{l}\text { Cross- } \\
\text { sectional }\end{array}$ & $\begin{array}{l}\text { U.S. population- } \\
\text { Aged } 18 \text { years and } \\
\text { older } \\
\text { Both sexes } \\
\text { All races }\end{array}$ & $\begin{array}{l}\text { Assess } \\
\text { prevalence of } \\
\text { tobacco } \\
\text { exposure. } \\
\text { Compare } \\
\text { prevalence } \\
\text { rates with } \\
\text { self-reported } \\
\text { smoking }\end{array}$ & $\begin{array}{l}\text { Unexposed }=<0.05 \\
\mathrm{ng} / \mathrm{mL} \\
\text { Light SHS exposure } \\
=0.05-0.49 \mathrm{ng} / \mathrm{mL} \\
\text { Significant SHS } \\
\text { exposure }=0.5-13.9 \\
\mathrm{ng} / \mathrm{mL} \\
\text { Active smoking }=\geq 14 \\
\text { ng/mL) }\end{array}$ \\
\hline $\begin{array}{l}\text { Benowitz } \\
\text { et al. } \\
(2008)\end{array}$ & $\begin{array}{l}\text { Cross- } \\
\text { sectional }\end{array}$ & $\begin{array}{l}\text { U.S. population } \\
\text { (NHANES)- } \\
\text { Aged } 12 \text { years and } \\
\text { older } \\
\text { Both sexes } \\
\text { All races }\end{array}$ & $\begin{array}{l}\text { Determine } \\
\text { optimal cut- } \\
\text { points for } \\
\text { distinguishin } \\
\text { g smokers } \\
\text { from non- } \\
\text { smokers }\end{array}$ & $\begin{array}{l}\text { Smoker vs } \\
\text { nonsmoker } \\
\text { Adult }=3.08 \mathrm{ng} / \mathrm{mL} \\
\text { Adolescent= } 2.99 \mathrm{ng} / \mathrm{m} \\
1 \\
\text { Females }=4.47 \mathrm{ng} / \mathrm{mL} \\
\text { Males }=1.78 \mathrm{ng} / \mathrm{mL} \\
\text { AA }=5.92 \mathrm{ng} / \mathrm{mL} \\
\text { NHW }=4.85 \mathrm{ng} / \mathrm{m} \\
\text { Mexican Americans } \\
=0.84 \mathrm{ng} / \mathrm{mL}\end{array}$ \\
\hline
\end{tabular}




\section{Smoking and Ovarian reserve}

The association of smoking with ovarian reserve have been largely inconsistent. Of the thirteen epidemiological studies that have explored the association of smoking with ovarian reserve, only eight found a significant association. Of the thirteen studies, only one used the biomarker cotinine to validate smoking status among participants [20].

Fuentes et al. in a prospective cohort study examined the association between cigarette smoking and AMH concentrations in follicular fluid of fifty-six women undergoing first ART cycle [20]. Smoking status was based on follicular cotinine level and self-report. Women with cotinine concentrations $>0.25 \mathrm{ng} / \mathrm{mL}$ in the follicular fluid were considered cotinine positive otherwise subjects were classified as cotinine negative. Participants were also categorized into current smokers, ex-smokers, and never smokers based on response to the smoking questionnaire. The mean concentration of AMH in follicular fluid was significantly decreased in the cotinine positive group compared to the cotinine negative group $(1.02 \pm 0.14$ and $1.74 \pm 0.15$, respectively, $\mathrm{p}<0.05)$ [20]. Similarly, current smokers had significantly lower mean AMH level $(0.90 \pm 0.08 \mathrm{ng} / \mathrm{ml})$ compared to ex-smokers $(2.00 \pm 0.23 \mathrm{ng} / \mathrm{ml})$ and never smokers $(1.68 \pm 0.18 \mathrm{ng} / \mathrm{ml})$ [20]. A similar association was also found by the author in a follow up study of 141 infertile women [93]. Active smoking was significantly associated with a $2.29 \mathrm{ng} / \mathrm{mL}$ decrease in plasma AMH levels (p<0.001) [93].

Another prospective study of 277 women undergoing IVF found decreased levels of basal serum AMH among smokers compared to non-smokers ( $3.57 \pm 1.74$ vs. $4.34 \pm$ 1.91). In addition, among women that received antagonist ovarian stimulation protocol, smokers had significantly lower AMH levels $(2.86 \pm 1.32 \mu \mathrm{g} / \mathrm{l})$ compared to non-smokers 
$(3.74 \pm 1.69 \mu \mathrm{g} / \mathrm{l})[21]$. Smoking status was based on self-report and women that quit for more than a year were considered non-smokers.

Schuh-Huerta et al. prospectively examined the effects of environmental or lifestyle factors including self-reported smoking status on AMH levels among multiethnic cohort of women aged $25-45$ years [16]. An inverse association between smoking and AMH levels was found. After controlling for age, serum AMH levels were significantly higher among smoking women $(33.5 \pm 2.4)$ compared to non-smoking women $(27.4 \pm 1.7 \mathrm{pM})(\mathrm{p}=0.038)[16]$. The mean AMH level was found to be significantly lower $(\mathrm{p}<0.001)$ in African-American women compared to Caucasians. Mean AMH level was $30.1 \pm 1.5 \mathrm{pM}$ in Caucasians and $22.8 \pm 1.7 \mathrm{pM}$ in African Americans [16].

Self-reported active smoking was found to be associated with a lower serum AMH concentrations in a retrospective analysis of IVF patients. Serum AMH concentrations were significantly lower in the smoker group $(3.06+/-1.68 \mu \mathrm{g} / \mathrm{l})$ compared to non-smokers $(3.86+/-1.92 \mu \mathrm{g} / \mathrm{l})$ after controlling for age and BMI [17]. In addition, serum AMH level was negatively correlated with the number of cigarettes smoked daily $(\mathrm{r}=-0.36, \mathrm{p}<0.001)[17]$.

In a cross-sectional study of 284 women aged 38 to 50 years an association between current smoking and lower AMH levels was observed [94]. Based of questionnaire response, women were classified as current smokers; past smokers-reported a history of smoking, with cessation occurring at least 2 years before the study; passive smokers-currently living with someone who smoked in their home or never smokers. Only current smoking was associated with lower AMH values. Current smokers had 44\% 
lower AMH values than Never smokers $(p=0.04)$ [94]. However, the impact of smoking on $\mathrm{AMH}$ values was not dose dependent based on cigarettes per day $(\mathrm{p}=0.08)$ or packyears $(p=0.22)[94]$.

Caserta et al. in a cross-section of 296 infertile women observed a significantly reduced AFC among women who reported current smoking $(\mathrm{p}=0.04)$ [22]. In addition, a negative correlation was found between AFC and number of pack-years $(r=-0.32, p=$ $0.001)[22]$.

White et al. found similar inverse association of smoking with measures of ovarian reserve in a cross-section of 913 premenopausal women enrolled in the Sister study cohort [95]. Current heavy smoking, defined as smoking 20 or more cigarettes per day, was associated with reduced AMH (-55.3\%, 95\% CI: -79.8, -0.9) [95]. Among women who are 48 years and younger, both heavy smoking $(-71.4 \%, 95 \% \mathrm{CI}:-87.9$, $-32.4)$ and high pack-years $(-63.4 \%, 95 \% \mathrm{CI}:-83.5,-18.7)$ was associated with reduced AMH in current smokers [95].

Not all studies found an association between active smoking and AMH levels. In a prospective study of 136 normo-ovulatory women undergoing infertility work-up, median plasma AMH concentrations and AFC were not significantly different between self-reported smokers and nonsmokers [55]. In a multiple regression analysis adjusting for age smoking was not significantly association with either AMH $(\mathrm{p}=0.41)$ or AFC $(\mathrm{p}=0.34)[55]$.

In a retrospective data analysis on the effect of previous or current cigarette smoking on risk of premature ovarian failure, there was no statistically significant difference in mean serum AMH concentration between the smoking categories (current, 
ex-smoker and non-smoker) after adjustment for age (p-value 0.895) [96]. Hawkins

Bressler et al. in cross-sectional study of 1654 African-American women aged 23-34 found no association between self-reported active smoking and serum AMH concentrations [97]. In two other cross-sectional studies of premenopausal women, smoking was not significantly associated with measures of ovarian reserve $[12,98]$. 
Table 3: Summary of Studies on Active Smoking and AMH Levels

\begin{tabular}{|c|c|c|c|c|c|c|}
\hline Authors & $\begin{array}{l}\text { Study } \\
\text { population }\end{array}$ & $\begin{array}{l}\text { Study } \\
\text { design }\end{array}$ & Exposure & Outcome & Confounder & Results \\
\hline $\begin{array}{l}\text { Jung et } \\
\text { al. } \\
\text { (2017) }\end{array}$ & $\begin{array}{l}\text { Premenopausal } \\
\text { women (9 } \\
\text { cohorts in U.S } \\
\text { and Europe) } \\
\mathrm{N}=671\end{array}$ & $\begin{array}{l}\text { Cross- } \\
\text { sectional }\end{array}$ & $\begin{array}{l}\text { Self-reported } \\
\text { smoking } \\
\text { Prevalence of } \\
\text { smoking=20.3\% }\end{array}$ & $\mathrm{AMH}$ & $\begin{array}{l}\text { Age, current } \\
\text { use of OCP, } \\
\text { age at } \\
\text { menarche }\end{array}$ & $\begin{array}{l}\text { No Association } \\
\text { P-value }=0.73\end{array}$ \\
\hline $\begin{array}{l}\text { Hawkins } \\
\text { et al. } \\
\text { (2016) }\end{array}$ & $\begin{array}{l}\text { African- } \\
\text { American } \\
\text { women 23-34 } \\
\text { years (Detroit, } \\
\text { MI) } \\
\mathrm{N}=1654\end{array}$ & $\begin{array}{l}\text { Cross- } \\
\text { sectional }\end{array}$ & $\begin{array}{l}\text { Self-reported } \\
\text { smoking } \\
\text { Prevalence of } \\
\text { smoking }=19 \%\end{array}$ & $\begin{array}{l}\text { Serum } \\
\text { AMH }\end{array}$ & $\begin{array}{l}\text { Age, BMI, } \\
\text { hormonal } \\
\text { contraception }\end{array}$ & $\begin{array}{l}\text { No Association \% } \\
\text { change in AMH } \\
(95 \% \text { CI). } \\
\text { Current } \\
\text { smoker=1.9 (- } \\
11,17) \text { Former } \\
\text { smoker=7.5 (- } \\
\text { 12,32) Never- } \\
\text { smoker=Referent }\end{array}$ \\
\hline $\begin{array}{l}\text { Kline et } \\
\text { al. } \\
(2016)\end{array}$ & $\begin{array}{l}\text { Premenopausal } \\
\text { U.S. women } \\
\text { (New Jersey) } \\
\mathrm{N}=477\end{array}$ & $\begin{array}{l}\text { Cross- } \\
\text { sectional }\end{array}$ & $\begin{array}{l}\text { Self-reported } \\
\text { smoking } \\
\text { Prevalence of } \\
\text { smoking }=10.9 \%\end{array}$ & $\begin{array}{l}\text { Serum } \\
\text { AMH }\end{array}$ & Age & $\begin{array}{l}\text { No Association } \\
\text { Mean } \\
\text { difference(95\% CI) } \\
\text { in LnAMH } \\
\text { Former smoker= } \\
\text { 0.111 } \\
(-0.107,0.329) \\
\text { Current smoker= } \\
-\mathbf{0 . 2 2 3} \\
(-\mathbf{0 . 5 3 9 , 0 . 0 9 4 )}\end{array}$ \\
\hline $\begin{array}{l}\text { White et } \\
\text { al. } \\
(2016)\end{array}$ & $\begin{array}{l}\text { Premenopausal } \\
\text { U.S women } \\
\text { enrolled in } \\
\text { Sister study } \\
\mathrm{N}=913\end{array}$ & $\begin{array}{l}\text { Cross- } \\
\text { sectional }\end{array}$ & $\begin{array}{l}\text { Self-reported } \\
\text { smoking } \\
\text { Prevalence of } \\
\text { smoking }=8.4 \%\end{array}$ & $\begin{array}{l}\text { Serum } \\
\text { AMH }\end{array}$ & $\begin{array}{l}\text { Age, parity, } \\
\text { breastfeeding } \\
\text { history, race, } \\
\text { education } \\
\text { and annual } \\
\text { household } \\
\text { income }\end{array}$ & $\begin{array}{l}\text { Association } \\
\text { \% change in AMH } \\
(95 \% \text { CI }) \\
\text { Nonsmokers- } \\
\text { referent } \\
<15 \\
\text { cigarettes/day= } \\
89.6 \\
(-10.5,301.7) \\
15-19 \\
\text { cigarettes/day = - } \\
21.9 \\
(-66.1,80.3) \\
20+ \\
\text { cigarettes/day= - } \\
\mathbf{5 5 . 3} \\
(-79.8,-0.9)\end{array}$ \\
\hline
\end{tabular}


Table 3: Summary of Studies on Active Smoking and AMH Levels Continuation

\begin{tabular}{|c|c|c|c|c|c|c|}
\hline Authors & $\begin{array}{l}\text { Study } \\
\text { population }\end{array}$ & $\begin{array}{l}\text { Study } \\
\text { design }\end{array}$ & Exposure & Outcome & Confounder & Results \\
\hline $\begin{array}{l}\text { Caserta et } \\
\text { al. (2013) }\end{array}$ & $\begin{array}{l}\text { Romanian } \\
\text { women } \\
\text { seeking } \\
\text { fertility } \\
\text { treatment } \\
\mathrm{N}=296\end{array}$ & $\begin{array}{l}\text { Cross- } \\
\text { sectional }\end{array}$ & $\begin{array}{l}\text { Self- } \\
\text { reported } \\
\text { smoking } \\
\text { Prevalence } \\
\text { of } \\
\text { smoking=34 } \\
.5 \%\end{array}$ & Day 3 AFC & None & $\begin{array}{l}\text { Association } \\
\text { AFC } \\
\text { Nonsmoker } \\
\text { s= 10.1 } \pm \\
3.9 \\
\text { Smokers= } \\
9.0 \pm 4.1 \\
\text { P-value= } \\
\text { 0.04 }\end{array}$ \\
\hline $\begin{array}{l}\text { Fuentes et } \\
\text { al. (2013) }\end{array}$ & $\begin{array}{l}\text { Chilean } \\
\text { women } \\
\mathrm{N}=141\end{array}$ & $\begin{array}{l}\text { Prospective } \\
\text { cohort }\end{array}$ & $\begin{array}{l}\text { Self- } \\
\text { reported } \\
\text { smoking } \\
\text { Prevalence } \\
\text { of } \\
\text { smoking=23 } \\
\%\end{array}$ & $\mathrm{AMH}$ & Age, BMI & $\begin{array}{l}\text { Association } \\
\mathbf{P}<\mathbf{0 . 0 0 1}\end{array}$ \\
\hline $\begin{array}{l}\text { Fuentes et } \\
\text { al. (2012) }\end{array}$ & $\begin{array}{l}\text { Chilean } \\
\text { women } \leq 38 \\
\text { years } \\
\text { undergoing } \\
1^{\text {st }} \text { IVF } \\
\text { treatment } \\
\mathrm{N}=56\end{array}$ & $\begin{array}{l}\text { Prospective } \\
\text { Cohort }\end{array}$ & $\begin{array}{l}\text { Self- } \\
\text { reported } \\
\text { active } \\
\text { smoking } \\
\text { and } \\
\text { follicular } \\
\text { cotinine } \\
\text { level } \\
\text { Prevalence } \\
\text { of } \\
\text { smoking=28 } \\
.6 \%\end{array}$ & $\begin{array}{l}\text { AMH } \\
\text { concentratio } \\
\text { ns in } \\
\text { follicular } \\
\text { fluid }\end{array}$ & Age, BMI & $\begin{array}{l}\text { Association } \\
\text { Mean } \\
\text { AMH } \pm \text { s.d } \\
(\text { ng/ml) } \\
\text { Current } \\
\text { smoker }=0.9 \\
0 \pm 0.08 \\
\text { Ex- } \\
\text { smoker=2.0 } \\
0 \pm 0.23 \\
\text { Never } \\
\text { smoker=1.6 } \\
8 \pm 0.18 \\
\text { Cotinine } \\
\text { positive } \\
(>0.25) \\
=1.02 \pm 0.14 \\
\text { Cotinine } \\
\text { negative }=1 . \\
74 \pm 0.15 \\
\end{array}$ \\
\hline $\begin{array}{l}\text { Schuh- } \\
\text { Huerta et al. } \\
\text { (2012) }\end{array}$ & $\begin{array}{l}\text { U.S. women } \\
(25- \\
45 y e a r s) \\
\text { enrolled in } \\
\text { OVA study } \\
\text { (California) } \\
\mathrm{N}=432\end{array}$ & Cohort & $\begin{array}{l}\text { Self- } \\
\text { reported } \\
\text { active } \\
\text { smoking } \\
\text { Prevalence } \\
\text { of smoking } \\
\text { NHW=14.6 } \\
\% \text {; } \\
\text { AA=11.4\% }\end{array}$ & $\begin{array}{l}\text { Serum } \\
\text { AMH }\end{array}$ & Age & $\begin{array}{l}\text { Association } \\
\text { Mean } \\
\text { AMH } \pm \text { s.d } \\
(\text { pM }) \\
\text { Smoker= } \\
33.5 \pm 2.4 \\
\text { Non- } \\
\text { smoker= } \\
27.4 \pm 1.7\end{array}$ \\
\hline
\end{tabular}


Table 3: Summary of Studies on Active Smoking and AMH Levels Continuation

\begin{tabular}{|c|c|c|c|c|c|c|}
\hline Authors & $\begin{array}{l}\text { Study } \\
\text { population }\end{array}$ & Study design & Exposure & Outcome & Confounder & Results \\
\hline $\begin{array}{l}\text { Ferour } \\
\text { et al. } \\
\text { (2012) }\end{array}$ & $\begin{array}{l}\text { French } \\
\text { women } \\
\text { undergoing } \\
\text { IVF } \\
\mathrm{N}=277\end{array}$ & $\begin{array}{l}\text { Prospective } \\
\text { cohort }\end{array}$ & $\begin{array}{l}\text { Self-reported } \\
\text { smoking } \\
\text { Prevalence of } \\
\text { smoking }=28.9 \%\end{array}$ & $\begin{array}{l}\text { Serum } \\
\text { AMH }\end{array}$ & $\begin{array}{l}\text { Age, BMI, } \\
\text { basal FSH, } \\
\text { basal LH, } \\
\text { basal } \\
\text { estradiol, } \\
\text { IVF rank, } \\
\text { amount of } \\
\text { recombinant } \\
\text { FSH } \\
\text { injected, } \\
\text { ICSI } \\
\text { assessed in } \\
\text { subgroup } \\
\text { analysis }\end{array}$ & $\begin{array}{l}\text { Association } \\
\text { Mean AMH } \pm \\
\text { s.d }(\mu \mathrm{g} / \mathrm{l}) \\
\text { Smoker }= \\
2.86 \pm 1.3 \\
\text { Non-smoker= } \\
3.74 \pm 1.69\end{array}$ \\
\hline $\begin{array}{l}\text { Plante et } \\
\text { al. } \\
(2010)\end{array}$ & $\begin{array}{l}\text { U.S. } \\
\text { women } 38- \\
50 \text { years } \\
\mathrm{N}=284\end{array}$ & $\begin{array}{l}\text { Cross- } \\
\text { sectional }\end{array}$ & $\begin{array}{l}\text { Self-reported } \\
\text { smoking } \\
\text { Prevalence of } \\
\text { smoking }=10.9 \%\end{array}$ & $\begin{array}{l}\text { Serum } \\
\text { AMH }\end{array}$ & Age, BMI & $\begin{array}{l}\text { Association } \\
\% \text { change in } \\
\text { AMH }(95 \% \mathrm{CI}) \\
\text { Current } \\
\text { smoker=-44 (- } \\
68,-2) \\
\text { Past smoker= -1 } \\
(-32,44) \\
\text { Never } \\
\text { smoker=referent }\end{array}$ \\
\hline $\begin{array}{l}\text { Waylen } \\
\text { et al. } \\
(2010)\end{array}$ & $\begin{array}{l}\text { British } \\
\text { women } \\
(24- \\
\text { 48years) } \\
\text { using test } \\
\text { of ovarian } \\
\text { reserve } \\
\mathrm{N}=335 \\
\end{array}$ & $\begin{array}{l}\text { Retrospective } \\
\text { data analysis }\end{array}$ & $\begin{array}{l}\text { Self-reported } \\
\text { active smoking } \\
\text { Prevalence of } \\
\text { smoking }=14.0 \%\end{array}$ & $\begin{array}{l}\text { Serum } \\
\text { AMH }\end{array}$ & Age & $\begin{array}{l}\text { No Association } \\
\text { p-value } 0.895\end{array}$ \\
\hline $\begin{array}{l}\text { Ferour } \\
\text { et al. } \\
(2008)\end{array}$ & $\begin{array}{l}\text { French } \\
\text { women } \\
\text { undergoing } \\
\text { IVF } \\
\mathrm{N}=111\end{array}$ & $\begin{array}{l}\text { Retrospective } \\
\text { analysis }\end{array}$ & $\begin{array}{l}\text { Self-reported } \\
\text { active smoking } \\
\text { Prevalence of } \\
\text { smoking=36\% }\end{array}$ & $\begin{array}{l}\text { Serum } \\
\text { AMH }\end{array}$ & Age, BMI & $\begin{array}{l}\text { Association } \\
\text { Mean AMH } \pm \\
\text { s.d }(\mu \mathrm{g} / \mathrm{l}) \\
\text { Smoker }=3.06 \pm \\
1.68 \\
\text { Non-smoker }= \\
\text { 3.86 } \pm 1.92\end{array}$ \\
\hline $\begin{array}{l}\text { Nardo et } \\
\text { al. } \\
(2007)\end{array}$ & $\begin{array}{l}\text { British } \\
\text { women } \\
\text { undergoing } \\
\text { IVF } \\
\mathrm{N}=136 \\
\end{array}$ & $\begin{array}{l}\text { Prospective } \\
\text { cohort }\end{array}$ & $\begin{array}{l}\text { Self-reported } \\
\text { smoking } \\
\text { Prevalence of } \\
\text { smoking=18\% }\end{array}$ & $\begin{array}{l}\text { Plasma } \\
\text { AMH } \\
\text { AFC }\end{array}$ & Age & $\begin{array}{l}\text { No Association } \\
\text { P-value } \\
\text { AMH=0.41 } \\
\text { P-value } \\
\text { AFC=0.34 } \\
\end{array}$ \\
\hline
\end{tabular}


A strong association between active smoking and ovarian reserve has not been established due to inconsistency in available data. Prior studies have been limited by small sample size [20], adjustment for a limited number of possible confounders [16, 17, $20,22,55,93,94,96,98]$ and use of self-report to assess exposure $[12,16,17,21,22$, 55, 93-98]. The categorization of smoking status also varied across the studies. Definition of smoking status was not explicitly stated $[12,16,17,21,22,55,96]$ and assessment of smoking status was based on three $[12,20,94-98]$ or two categories $[16,17,21,22,55$, 93] (e.g., never/past/current or never/ever) which did not consider the duration and quantity of smoking. The few studies that explored dose-response relationship between smoking and ovarian reserve assessed dose using number of cigarettes smoked [59, 95, 97], duration of smoking in years [95, 97] and pack-years of smoking [22, 59, 95].

\section{N-acetyltransferase 2 (NAT2)}

$\mathrm{N}$-acetyltransferase 2 (NAT2) is a polymorphic phase 2 metabolic enzyme that conjugates hydrazine derivatives and aromatic amine drugs with acetyl-groups [25, 99]. NAT2 is expressed primarily in the liver and gastrointestinal tract and involved in the acetylation and metabolism of some procarcinogens and xenobiotics [25, 26, 29, 100, 101]. The NAT2 enzyme plays important roles in the metabolic activation and deactivation of carcinogenic aromatic amine, 4-aminobiphenyl (ABP), found in cigarette smoke $[25,102]$. Studies of human 4-ABP exposure reported higher levels of 4-ABPDNA adducts that initiate mutagenesis and carcinogenesis [103], in smokers than nonsmoker in tissues such as larynx [104], liver [105], bladder [106, 107], breast [108111] and sputum [112]. The role of 4-ABP-DNA adducts and their relationship with tobacco exposure and ovarian reserve is yet to be evaluated. DNA adducts are a function 
of the environmental exposure and polymorphisms in genes involved in carcinogen metabolism [113].

Polymorphisms in the NAT2 gene results in biological differences in acetylating capacity and metabolism that form the basis for slow, intermediate, and rapid acetylator phenotypes [99-101, 114]. Various combinations of single nucleotide polymorphisms (SNPs) on the NAT2 gene are identified as NAT2 alleles or haplotypes and many NAT2 alleles have been identified in human populations $[100,115]$. The NAT2*4 allele considered the "wild-type" allele due to absence of SNPs, is associated with rapid acetylation activity $[26,29,114]$. The NAT2 alleles containing the rs 1801279 (191G>A), rs 1801280 (341T>C), rs1799930 (590G>A), and rs1799931 (857G>A) SNPs are associated with slow acetylation $[29,114,116]$. The most common slow acetylator alleles in human populations contain one or more of these polymorphisms, identified as $N A T 2 * 5, N A T 2 * 6, N A T 2 * 7$, and $N A T 2 * 14$ and their subtypes [26]. Individuals homozygous for "wild-type" NAT2 acetylator alleles are deduced as rapid acetylators, individuals homozygous for slow acetylator NAT2 alleles are deduced as slow acetylators, and individuals possessing one rapid and one slow NAT2 allele are deduced as intermediate acetylators $[29,114,117]$.

Ethnic differences in the frequency of rapid and slow acetylator NAT2 alleles or haplotype have been reported [24, 26, 29, 118-120]. The wild-type allele occurs at somewhat higher frequency (41-65\%) in African Americans than in non-Hispanic whites $(20-46 \%)[24,26,121]$. The slow acetylator alleles occur in about $40-60 \%$ of nonHispanic white population and African Americans [24, 26, 118, 120, 121]. The ethnic 
disparity in NAT2 acetylator status is attributed to ethnic differences in the frequencies of SNPs and genotypes [29].

Variable reductions in catalytic activity, substrate affinity, and stability of NAT2 protein expressed in human liver from individuals with slow acetylator phenotype have been reported $[29,100,101,114,122]$. Slow NAT2 acetylators have been shown to have higher serum concentrations of drugs and other xenobiotics, and increased susceptibility to adverse drug reactions and effects of toxins including those found in tobacco smoke $[25,26,100,101,114]$

Epidemiological studies have explored the association of NAT2 polymorphism with risk of various cancers such as urinary bladder [27, 29, 123-125], colorectal [126], lung [127], cervical [28], breast [128, 129] and ovarian [130]. NAT2 acetylator status also modified the association of smoking with risk of bladder and breast cancer, and colorectal adenoma $[27,125,126,128]$. The presence of slow acetylation increased the bladder cancer risk in smokers $(\mathrm{OR}, 1.51 ; 95 \% \mathrm{CI}, 1.34-1.70 ; \mathrm{p}<.00001)$ in a fixed-effect model including 9 studies (3412 cases (663 nonsmokers and 2749 smokers) and 3507 controls (1413 nonsmokers and 2094 smokers)) [27]. In postmenopausal slow acetylators, current and past smoking increased breast cancer risk in a dose-dependent manner [128]. The adjusted odds ratio for smoking $\leq 15,16$ to 20 and more than 20 cigarettes in past 2 years were 0.8 (95\% CI:0.3-2.5), 3.2 (95\% CI:1.3-7.8),4.4 (95\% CI:1.3-14.8), respectively (ptrend, <0.01) [128]. Additionally, packs per average year significantly elevated breast cancer risk among postmenopausal slow acetylators (OR,2.8; 95\% CI: 1.4-5.5) [128].

Only a handful of studies have assessed the effects of NAT2 acetylators on fertility and related outcomes in women. Mendola et al. in a case-control study (29 cases 
and 72 controls) investigated the effect of smoking, NAT2 genotype and their interaction on recurrent spontaneous abortion in women. The odds ratio for recurrent spontaneous abortion was 1.34 (95\% CI: 0.63 - 2.86) among smokers and 1.34 (95\% CI: 0.56 - 2.54) among slow acetylators [131]. No significant interaction was observed. A positive association between slow acetylator status and endometriosis was reported in case-control study (29 cases and 72 controls) conducted in France [132]. In a prospective study of 319 women office workers followed for an average of 8 menstrual cycles, NAT2 acetylator status modified the effect of smoking on fecundability [24]. Current smoking was significantly associated with reduced fecundability among slow acetylators, with an adjusted fecundability odds ratio of 0.34 (95\% CI: $0.22-0.90)$ [24].

\section{Summary and Gap in Current Knowledge}

Genetic heterogeneity is one factor that may explain the inconsistent results of previous studies on the association of smoking with ovarian reserve. NAT2 acetylator status have been shown to modify the association of smoking with different disease outcomes, with slow acetylators being more susceptible [23, 24, 27, 125, 126, 131]. Taylor et al. reported reduced fecundability among current smokers who are slow acetylators [24]; however, it remains to be established if the interaction of NAT2 genotype and smoking on fecundability is modulated through reduction in ovarian reserve. No studies have assessed the effect of NAT2 acetylator status or its' potential interaction with smoking on ovarian reserve.

African- American women have lower ovarian reserve as measured by AMH levels, than NHW women, a finding that has been attributed primarily to genetic variations [16]. However, life style factor such as smoking appears to play a significant role, but to date 
there have been no well-designed studies to address this issue. It is unclear to what extent ethnicity may interact with smoking to affect ovarian reserve. None of the studies on the association of smoking with ovarian reserve evaluated racial differences; most of the studies were restricted to a single ethnic group. Only a handful included NHW and AA participants; however, racial disparity in association of smoking with AMH were not evaluated.

This study will not only add to the body of evidence on the relationship between smoking and ovarian reserve, it will provide evidence-based information about the magnitude and reliability of associations between active smoking and ovarian reserve with better validation of exposure using cotinine measurement.

Findings from this study will contribute to the understanding of the racial disparity in ovarian reserve and how environmental/behavioral risk factors and genetic susceptibility may interact to reduce a woman's ovarian reserve, impacting her reproductive potential. Findings will also provide evidence to support smoking cessation and policies to reduce exposure to tobacco smoke. 


\section{RESEARCH DESIGN AND METHODS}

\section{Study Design}

Given the uncertainty as to the relationship of smoking with ovarian reserve and the possibility for a long latency period, a cross-sectional study nested within the ongoing Louisville Tobacco Smoke Exposure, Genetic Susceptibility \& Infertility Study (LOUSSI study) was used. This study design allowed comparison of the prevalence of smoking among women with diminished ovarian and women with normal ovarian reserve, estimation of the association of smoking with ovarian reserve and evaluation of the interaction of smoking with race/ethnicity and NAT2 genotype. Other possible risk factors were also evaluated in relation to ovarian reserve.

The cross-sectional study design offered the advantage of a shorter study duration, no loss to follow and reduce cost. This is especially important, as little is known about the time frame from exposure to tobacco smoke and the development of DOR.

\section{Study Population}

The Louisville Tobacco Smoke Exposure, Genetic Susceptibility \& Infertility Study (LOUSSI study) is an observational study that is focused on the effects of 
tobacco exposure and NAT2 acetylator status on ovarian reserve and IVF outcomes (Granting institution- Eunice Kennedy Shriver National Institute of Child Health and Human Development; grant number- 1R15HD087911-01; principal investigator-Dr. Kira Taylor). Institutional Review Board approval for this study was obtained from the University of Louisville, IRB number:16.0063.

Participants were recruited from the infertility clinic at the University of Louisville Reproductive Endocrinology and Infertility (REI) clinic. These participants are women of reproductive age seeking infertility treatment and were mostly from the Louisville and southern Indiana area. Based on medical record data and personal communication with Dr. Bohler (Co-investigator on LOUSSI study), about 350 female patients were referred to the University of Louisville REI clinic for fertility treatment in 2017 and approximately 55\% are Non-Hispanic White, 27\% Non-Hispanic Black and $10 \%$ are Hispanic. Data from CDC Assisted Reproductive Technology (ART) report showed that $60 \%$ of women who used ART at the University of Louisville Fertility center in 2015 were less than 35 years of age [133].

Inclusion Criteria: All women 21 years and above seeking infertility treatment at the University of Louisville Reproductive Endocrinology and Infertility (REI) clinic were eligible for enrollment into the study. Both new and existing patients planning various types of fertility treatments were eligible for recruitment into the study.

Excluded were women with ongoing pregnancy and patients who cannot communicate in English or are unable to understand and complete the informed consent and questionnaire. 


\section{Subject Recruitment Methods}

All clinic patients were mailed a participation letter and a brochure describing the study, inviting them to contact the Principal investigator if they wish to participate. In addition, women 21 years and older seeking fertility treatment at the REI clinic were approached during their visit by the attending physician to determine interest and eligibility for the study. Patients who express interest were referred to a research assistant who explains the benefits and risks of the study as well as the study procedures. Informed consent process (IRB number: 16.0063) was initiated for patients that agreed to participate and a gift card (\$25) offered, to thank them for their participation. For this dissertation, 265 women were recruited from the REI clinic between September 2016 and June 2018.

\section{Exposure Assessment}

The primary exposure, current active smoking, was assessed based on response to supplemental smoking questionnaire and urinary cotinine levels. The urinary cut-point for discriminating smokers from nonsmokers was set at $\geq 100 \mathrm{ng} / \mathrm{mL}$ and validity of selfreported smoking was tested using a Kappa statistic.

- Current smoker: - defined as a person that self-identifies as currently smoking at least 1 cigarette/week or quit less than 1 month ago; AND has positive cotinine levels $(\geq 100 \mathrm{ng} / \mathrm{mL})$.

- Former smoker: - defined as a non-current smoker with history of smoking at least 1 cigarette/week or quit more than 1 month ago AND has cotinine levels <14 $\mathrm{ng} / \mathrm{mL}$. 
- Nonsmoker: - never smoked more than 1 cigarette/week AND has cotinine levels $<14 \mathrm{ng} / \mathrm{mL}$.

- Cumulative lifetime smoking: Pack-years smoked was calculated for current and former smokers by multiplying the number of packs smoked per day by the years of smoking.

\section{Outcome Measurement}

Assessment of ovarian reserve was based on baseline serum AMH levels. This measure of ovarian reserve was extracted from patients' medical record. Women routinely provide a serum sample for analysis of reproductive hormones, including AMH as part of the initial infertility workup, and have transvaginal ultrasound performed to assess follicular development and count antral follicles. The AMH assays were performed by Quest Diagnostics using chemiluminescence with lower limit of detection being $0.03 \mathrm{ng} / \mathrm{mL}$. AMH value below the lower limit of detection was assigned a value of $0.03 \mathrm{ng} / \mathrm{mL}$.

The cut-points of AMH for diagnosis of DOR range from 0.1 to over $2.5 \mathrm{ng} / \mathrm{ml}$ $[10,48,49]$. For this study, diminished ovarian reserve (DOR) was defined as having baseline serum AMH level less than 1ng/mL [97, 134].

\section{NAT2 Acetylator Status}

Four SNPs, rs1801279 (191G>A), rs1801280 (341T>C), rs1799930 (590G>A), and rs1799931 (857G >A) in the NAT2 coding region and their corresponding alleles and haplotypes were determined [29]. NAT2 variants for these SNPs are associated with slow acetylation [29]. The NAT2*4 allele is associated with rapid acetylation activity [29]. 
- Rapid acetylators: defined as possessing two of the NAT2 alleles associated with rapid acetylation activity $(\mathrm{NAT} 2 * 4)$

- Intermediate acetylators: defined as possessing one of the alleles associated with rapid acetylation activity and one allele associated with slow acetylation (NAT2*5, NAT2*6, NAT2*7, and NAT2*14).

- Slow acetylators: individuals that possessed two slow acetylation alleles (NAT2 $* 5$, NAT2 $* 6, \mathrm{NAT}_{2} * 7$, and NAT2 $* 14$ ) were classified as slow acetylators.

\section{Covariates}

The covariates assessed include but are not limited to sociodemographic characteristics (age, ethnicity); marital status; weight and height at enrollment for calculation of body mass index (BMI); age at menarche, parity and PCOS status. Age, ethnicity, marital status, age at menarche and parity was based on self-report. PCOS status was based on self-report or physician diagnosis.

BMI at enrollment, calculated as the body weight in kilograms divided by the square of height in meters $\left(\mathrm{kg} / \mathrm{m}^{2}\right)$ was used to classify participants as normal weight (17.5 - 24.9), overweight (25.0 - 29.9), obese (30.0 - 34.9), or morbidly obese $(\geq 35)$ based on WHO classification [135].

\section{Data Collection and Study Procedures}

Exposure to tobacco smoke was assessed using a combination of a selfadministered smoking questionnaire (SSQ; appendix 1) and cotinine levels in urine. The SSQ was administered during subject's clinic visit. Urine sample was also collected for cotinine assay and DNA extraction, after informed consent was signed. Information on 
outcome measures and covariates were extracted from medical record (data collection form; appendix 2).

Medical record excerpts, biological specimens (urine and DNA samples) and questionnaires were labeled with an identification number randomly assigned to each participant. The newly assigned identification number was used to create and populate an electronic database with data from medical records, SSQ, cotinine and NAT2 assays.

1. Instrument: Self-administered Smoking Questionnaire (SSQ)

Once a participant has agreed to partake in the study and signed informed consent form, a self-administered SSQ designed to assess active and passive exposure to tobacco smoke was given to subject.

Quality control: Questions from the smoking questionnaire (SSQ) were adapted from smoking questions from National Health and Nutrition Examination Survey III (NHANES III) and National Health interview survey [136, 137].

\section{Medical Record Extraction}

Information on the outcome measure and important covariates were extracted from the patient history form (appendix 3) and medical record using data collection sheets labeled with the patient's randomly assigned identification number.

The patient history form is a survey completed by patients at first clinic visit. The survey is designed to collect information on demographic characteristics, reproductive and social history. Data extracted were selected based on the relationship to fertility outcomes, tobacco smoke exposure, or both. Information assessed from the patient history form include but are not limited to, date of visit; sociodemographic characteristics (age, ethnicity); menstrual history (age at first period, menstrual cycle length and 
regularity, ovulation); number of previous pregnancies; sexually transmitted infections; social history (occupation, marital status and length of time with current partner); physical exercise and diet history; alcohol and caffeine use; and smoking history.

The racial categories noted on the patient history form include American Indian; Asian; White; Alaska Native; Black or African American; Native Hawaiian or other Pacific Islander; and other. For ethnicity, patient can self-identify as Hispanic/ Latino or not Hispanic/Latino.

Information on $\mathrm{AMH}$ which is routinely done as part of infertility workup was extracted from the medical record as well as PCOS diagnosis. Results of AMH assay done after first clinic visit (for new patients) and within six months of enrollment (for existing patients) was extracted. Physical measurements (weight, height, BMI and blood pressure) taken at time of enrollment was also assessed from the medical record.

Quality control: Medical records were extracted in duplicate and updated as study progressed.

\section{Cotinine Assays}

Cotinine, a biomarker of tobacco use, is a major metabolite of nicotine and can be detected in urine and other bodily fluids for up to 72 hours following exposure [88].

Urine was collected at enrollment (during the patient's office visit) into sterile vials and labeled with the patient's randomly assigned identification number. Urine samples were divided into two; one for the cotinine assays and the other for DNA extraction. The samples were stored at 4 degrees Celsius until time of assay. DNA samples were frozen at -20 degrees Celsius until genotyping. 
Cotinine ELISA assays (Calbiotech, Spring Valley, CA) was used be used to estimate the level of cotinine in the urine of participants. The Calbiotech Cotinine kit is a solid phase competitive ELISA that contain standards and controls. Cotinine in the samples competes with a cotinine enzyme (HRP) conjugate for binding sites. The intensity of color generated at the end of assay procedure is inversely proportional to the concentration of cotinine in the samples. A standard curve was then prepared relating color intensity to the concentration of the cotinine. The maximum detectable level of cotinine for the Calbiotech assay is $100 \mathrm{ng} / \mathrm{mL}$. Urinary cotinine levels were estimated to the nearest $0.50 \mathrm{ng} / \mathrm{mL}$.

Quality control: All cotinine assays were done in triplicate and mean value was used. Samples with relative standard deviation greater than 0.10 were assayed in triplicate a second time in a subsequent assay.

\section{NAT2 Assays}

Genomic DNA was isolated from urine sample using the ZR Urine DNA Isolation $\mathrm{Kit}^{\mathrm{TM}}$ (Zymo Research, Irvine, CA. USA) according to manufacturer's instructions. A NAT2 four-SNP genotype panel of rs1801279 (191G>A), rs1801280 (341T>C), rs $1799930(590 \mathrm{G}>\mathrm{A})$ and $\mathrm{rs} 1799931(857 \mathrm{G}>\mathrm{A})$ was used to infer NAT2 acetylator phenotype. The accuracy of the four-SNP panel in determining NAT2 acetylator status is 98.4\% and comparable to the seven-SNP panel [138]. The assay uses SNP-specific PCR primers and fluorogenic probes designed using Primer Express ${ }^{\mathrm{TM}}$ (Applied Biosystems, CA, USA). The fluorogenic probes are labeled with a reporter dye (either carboxyfluorescein or VIC ${ }^{\circledR}$, Applied Biosystems) and are specific for one of the two 
possible bases identified at the four SNPs rs1801279 (191G>A), rs1801280 (341T>C), rs $1799930(590 \mathrm{G}>\mathrm{A})$ and $\mathrm{rs} 1799931(857 \mathrm{G}>\mathrm{A})$ in the NAT2 coding region [139].

The presence of the four SNPs was determined using a predeveloped TaqMan ${ }^{\circledR}$ SNP Genotyping Assays (Applied Biosystems) according to manufacturer's instructions. Briefly the $20 \times$ primer and probe mix was used at a $1 \times$ concentration with a half volume of 2× TaqMan Universal PCR Master Mix and $12.0 \mathrm{ng}$ of genomic sample per well [138, 140]. Assays were designed and optimized to work with TaqMan® Universal PCR Master Mix using the same thermal cycling conditions, which are $60^{\circ} \mathrm{C}$ for $30 \mathrm{~s}, 95^{\circ} \mathrm{C}$ for $10 \mathrm{~min}$ followed by 40 cycles of $95^{\circ} \mathrm{C}$ for $15 \mathrm{~s}$ and $60^{\circ} \mathrm{C}$ for $1 \mathrm{~min}$. The PCR amplification and end-point reading were performed on Step One Plus ${ }^{\mathrm{TM}}$ real-time instrument (Applied Biosystems). Major advantages of the TaqMan ${ }^{\circledR}$ SNP Genotyping Assays are that they do not require post-PCR processing (such as enzyme digestion) or the use of radioactivity [140].

Quality control: Controls (no DNA template) were ran to ensure that there is no amplification of contaminating DNA.

\section{Statistical Analyses}

Distribution of variables were examined; frequencies and percentages for categorical variables; mean and standard deviation for normally distributed continuous variable; median with interquartile ranges for non-normally distributed continuous variable. Differences in demographics and covariates between smokers and nonsmokers were tested using chi-square for categorical variables, Student's T-test for continuously normally distributed variable and Wilcoxon rank-sum for continuous variable not normally distributed. 
The outcome measure, baseline AMH level was not normally distributed. Two regression models, linear regression and logistic regression, was used to examine the relationship between active exposure to tobacco smoke and ovarian reserve.

Linear regression models with natural logarithm(ln) of AMH as the dependent variable was used to generate adjusted estimates of $\beta$ associated with each exposure of interest. Percent differences and 95\% confidence intervals (CIs) in serum AMH between exposure groups was calculated from the $\beta$ s as follows; $\left([\exp (\beta)-1]^{*} 100\right)$ and presented with corresponding 95\% confidence intervals (CIs) and p-values [97]. Multivariableadjusted logistic regression was used to assess odds of diminished ovarian reserve (DOR), defined as AMH $<1 \mathrm{ng} / \mathrm{mL}$, and $95 \%$ CIs.

Confounders were evaluated using a combination of theory and data-based approach. Adjustment were made for known covariates such as age, race/ethnicity, BMI, age at menarche parity and PCOS status in multivariable regression analyses while other variables that change the beta estimate for exposure by more than $10 \%$ were considered as confounder. In addition, regression analyses were stratified by PCOS status.

All analyses were two-sided and conducted using Statistical Analysis Software version 9.4 (SAS Institute, Cary, NC). Statistical significance was assumed for p-value $<0.05$.

Treatment of exposure variables for each of the specific aims:

Specific aim 1(a) Current active smoking (dichotomous; yes/no): The effect of current active smoking on ovarian reserve was assessed by comparing current active smokers with non-current smokers (Former smoker or never-smoker with cotinine levels $<14 \mathrm{ng} / \mathrm{mL})$. 


\section{Specific aim 1(b) dose-response relationship (Continuous and categorical):}

The dose of exposure for current active smoking was based on number of cigarettes smoked per day reported on the SSQ. The dose-response relationship between current active smoking and ovarian reserve was modeled as both a continuous exposure variable and categorical variable; 0 (nonsmoker), $1-<10$ cigarettes per day vs $\geq 10$ cigarettes per day.

\section{Specific aim 1(c) cumulative lifetime smoking (Continuous and categorical):}

The cumulative lifetime smoking was based on number of packs smoked per day and duration of smoking in years reported on the SSQ by both current active smokers and former smokers. The lifetime pack-years of smoking was calculated by multiplying the number of years reported smoking times the number of packs on average that they smoke per day. The effect of cumulative lifetime years of active smoking was assessed with both a continuous exposure variable and categorical variable using meaningful cut points.

Specific aim 2 Race - smoking interaction: To determine if the relationship measured in specific aim 1a differ by race, an interaction term for race and current smoking status was added to the model. The association was compared among nonHispanic White and African-Americans for potential effect modification. Information on self-reported race was extracted from medical record.

Specific aim 3 NAT2 acetylator status - smoking interaction: Intermediate and rapid acetylators were grouped together in the analysis because they have been shown to have similar acetylation activity. Rapid acetylators were compared to slow acetylators. NAT2, a dichotomous variable indicating slow/fast acetylator phenotype was created. 
The interaction term for smoking*NAT2 was included into the model to assess effect modification.

Model statement:

\section{Specific aim 1(a):}

Logistic regression:

$\log ($ odds of $D O R)=\beta 0+\beta 1 *$ Smoking $+\beta 2 *$ Age $+\beta 3 *$ Race $+\beta 4 * B M I+\beta 5 *$ Parity + $\beta 6 *$ Age at menarche $+\beta 7 * P C O S$

Linear regression:

$\operatorname{Ln}(A M H)=\beta 0+\beta 1 *$ Smoking $+\beta 2 *$ Age $+\beta 3 *$ Race $+\beta 4 * B M I+\beta 5 *$ Parity $+\beta 6 *$ Age at menarche $+\beta 7 * P C O S$

\section{Specific aim 2:}

Logistic regression:

$\log ($ odds of DOR $)=\beta 0+\beta 1 *$ Smoking $+\beta 2 *$ Race $+\beta 3 *$ Smoking $*$ Race $+\beta 4 *$ Age + $\beta 5 * B M I+\beta 6 *$ Parity $+\beta 7 *$ Age at menarche $+\beta 8 * P C O S$

Linear regression:

$\operatorname{Ln}(A M H)=\beta 0+\beta 1 *$ Smoking $+\beta 2 *$ Race $+\beta 3 *$ Smoking $*$ Race $+\beta 4 *$ Age $+\beta 5 * B M I+$ $\beta 6 *$ Parity $+\beta 7 *$ Age at menarche $+\beta 8 *$ PCOS

\section{Specific aim 3:}

Logistic regression:

$\log ($ odds of $D O R)=\beta 0+\beta 1 *$ Smoking $+\beta 2 * N A T 2+\beta 3 *$ Smoking $* N A T 2+\beta 4 *$ Age + $\beta 5 *$ Race $+\beta 6 * B M I+\beta 7 *$ Parity $+\beta 8 *$ Age at menarche $+\beta 9 * P C O S$ 


\section{Sample Size / Power calculation}

Based on the literature review, the estimated mean difference in AMH between current smokers and nonsmokers ranges between 0.78- 1.1 and the sample size estimates generated based on these effect sizes ranged from 118 -398.

Because parameters of diminished ovarian reserve and study population vary from study to study, sample size estimates and power calculations were based on preliminary data from the LOUSSI study. The estimated difference in mean AMH between current smokers and nonsmokers was $1.49 \mathrm{ng} / \mathrm{ml}$ with standard deviation of $4.18 \mathrm{ng} / \mathrm{mL}$. The estimated age- adjusted odds ratio for DOR for smoking 1 cigarette per day was $1.11(95 \%$ CI: $1.01-1.21, \mathrm{p}=0.03)$. The prevalence of smoking was $24 \%$ while the prevalence of DOR among non-smokers in this population was $30 \%$.

Two separate sample size calculations were performed for the main objective of the study, effect of smoking on ovarian reserve, using Proc power in SAS (SAS Institute, Cary, NC). The sample size estimates were based on statistical power of $80 \%$, alpha= 0.05 level of significance and ratio of smokers to nonsmokers set at 1:3.

Table 4.1 shows sample size estimates using a standard deviation of $4.18 \mathrm{ng} / \mathrm{mL}$ for varying mean differences in AMH when comparing smokers to nonsmokers. Sample size estimates for different odds ratio for DOR are presented in Table 4.2. Based on the Louisville Metro Behavioral Risk Factor Surveillance System, 24\% of women resident in Louisville metro were current smoker in 2016 [141]. The prevalence of smoking 10 cigarettes per day among the study population was assumed to be $11 \%$ and estimated odds ratio for DOR for women smoking 10 or more cigarettes per day is 2.84 (Table 4.2). 
Table 4. 1: Sample size estimate for the difference in mean AMH levels between smokers and nonsmokers

\begin{tabular}{|l|l|l|}
\hline $\begin{array}{l}\text { Mean difference in AMH } \\
(\mathrm{ng} / \mathrm{mL})\end{array}$ & Statistical power & Total sample size \\
\hline 1.4 & 0.801 & 376 \\
\hline 1.5 & 0.801 & 328 \\
\hline 1.6 & 0.800 & 288 \\
\hline 1.8 & 0.801 & 228 \\
\hline 2.0 & 0.807 & 188 \\
\hline
\end{tabular}

Table 4. 2: Sample size estimate for different odds ratio for effect of smoking on DOR

\begin{tabular}{|l|l|l|}
\hline Odds Ratio & Statistical Power & Total sample size \\
\hline 2.00 & 0.800 & 524 \\
\hline 2.50 & 0.802 & 282 \\
\hline $2.84(10$ cigarettes/day) & 0.803 & 210 \\
\hline 3.00 & 0.801 & 186 \\
\hline 3.50 & 0.803 & 138 \\
\hline 8.06 (for 1pack/day) & 0.805 & 42 \\
\hline
\end{tabular}

Power for active smoking and NAT2 interaction analyses were based on dichotomized exposure (smoker vs. nonsmoker) and outcome, DOR. Power calculations were performed for anticipated sample size of 250 using a power and sample size program for genetic association studies and gene-environment interaction, QUANTO (http://biostats.usc.edu/Quanto.html). Based on data from the LOUSSI study, the prevalence of current smoking and DOR in this population was $24 \%$ and $30 \%$, respectively. The estimated effect size for smoking and NAT2 was 1.4. Table 4.3 
provides power estimates for minimum detectable odds ratio for smoking-NAT2 interaction for an anticipated sample size of 250 at alpha $=0.05$ level of significance. The sample size is further broken down by NAT2 status in Table 4.4.

Table 4. 3: Power calculations for detecting a significant gene-environment interaction between active smoking and NAT2

\begin{tabular}{|l|l|l|}
\hline $\begin{array}{l}\text { Anticipated sample size } \\
\text { (40\% slow acetylators, 60\% } \\
\begin{array}{l}\text { Rapid/intermediate } \\
\text { acetylators) }\end{array}\end{array}$ & $\begin{array}{l}\text { Minimum detectable odds } \\
\text { ratio for smoking-NAT2 } \\
\text { interaction }\end{array}$ & $\begin{array}{l}\text { Power to detect significant } \\
\text { Smoking-NAT2 } \\
\text { interaction }\end{array}$ \\
\hline 250 & 1.4 & 0.17 \\
\hline 250 & 1.6 & 0.28 \\
\hline 250 & 1.8 & 0.41 \\
\hline 250 & 2.0 & 0.52 \\
\hline 250 & 2.5 & 0.74 \\
\hline
\end{tabular}

Table 4. 4: Sample size estimate stratified by NAT2 acetylator status

\begin{tabular}{|l|l|l|l|}
\hline NAT2 acetylator status & $\begin{array}{l}\text { Exposed } \\
\text { (smoker) }\end{array}$ & $\begin{array}{l}\text { Unexposed } \\
\text { (Nonsmokers) }\end{array}$ & Total \\
\hline Slow acetylators & 25 & 75 & 100 \\
\hline $\begin{array}{l}\text { Rapid/Intermediate } \\
\text { acetylators }\end{array}$ & 38 & 112 & 150 \\
\hline Total & 63 & 187 & 250 \\
\hline
\end{tabular}




\section{E. RESULTS}

Out of 265 women who were enrolled into the LOUSSI study, 59 (22.3\%) were excluded for the following reasons: missing medical record data on serum AMH levels $(n=44)$; or they did not provide urine for cotinine assays $(n=15)$. An additional 36 women were excluded from analyses due to lack of correlation between self-reported smoking status (never smoker or former smoker for more than 1 month) and increased cotinine level indicating either a high tobacco exposure $(\geq 100 \mathrm{ng} / \mathrm{mL}, \mathrm{n}=16)$ and or significant passive exposure (14-99ng/mL, n=20) (Figure 1). The overall Kappa statistics of selfreported smoking status was 0.81 (95\% CI: $0.72-0.89 ; \mathrm{p}<0.001)$ (data not shown).

The remaining 170 women included 31 (18\%) current active smokers and 139 (82\%) nonsmokers. Among the current smokers, 53\% reported smoking 10 or more cigarettes per day, $21 \%$ reported smoking between 5 to 9 cigarettes per day while $26 \%$ reported smoking less than 5 cigarettes per day.

Table 5.1 presents the characteristics of the women in the LOUSSI study stratified by smoking status. Nonsmokers had higher AMH levels, AFC and prevalence of DOR than smokers, but the differences were not significant. In general, smokers tended to be 
younger $(\mathrm{P}=0.04)$, of self-reported White race $(\mathrm{p}=0.01)$ and were more likely to be single ( $71 \%$ vs. $23 \%, \mathrm{p}=<0.001)$. Smokers and nonsmokers were comparable in terms of age at menarche, BMI, parity and prevalence of PCOS.

The NAT2 acetylator status of the participants is presented in Table 5.2. DNA extraction and/or NAT2 genotyping were unsuccessful in 14 participants. A total of 11 NAT2 genotypes were identified and assigned to rapid, intermediate and slow. About $39 \%$ of women in the study were slow acetylators, $44 \%$ intermediate and $17 \%$ rapid acetylators. Rapid and intermediate acetylators were merged for analysis. The distribution of NAT2 acetylator status did not differ by smoking status or self-reported race (Table $5.2)$. 
Figure 1. Flow Diagram for The Final Sample Size for Analysis

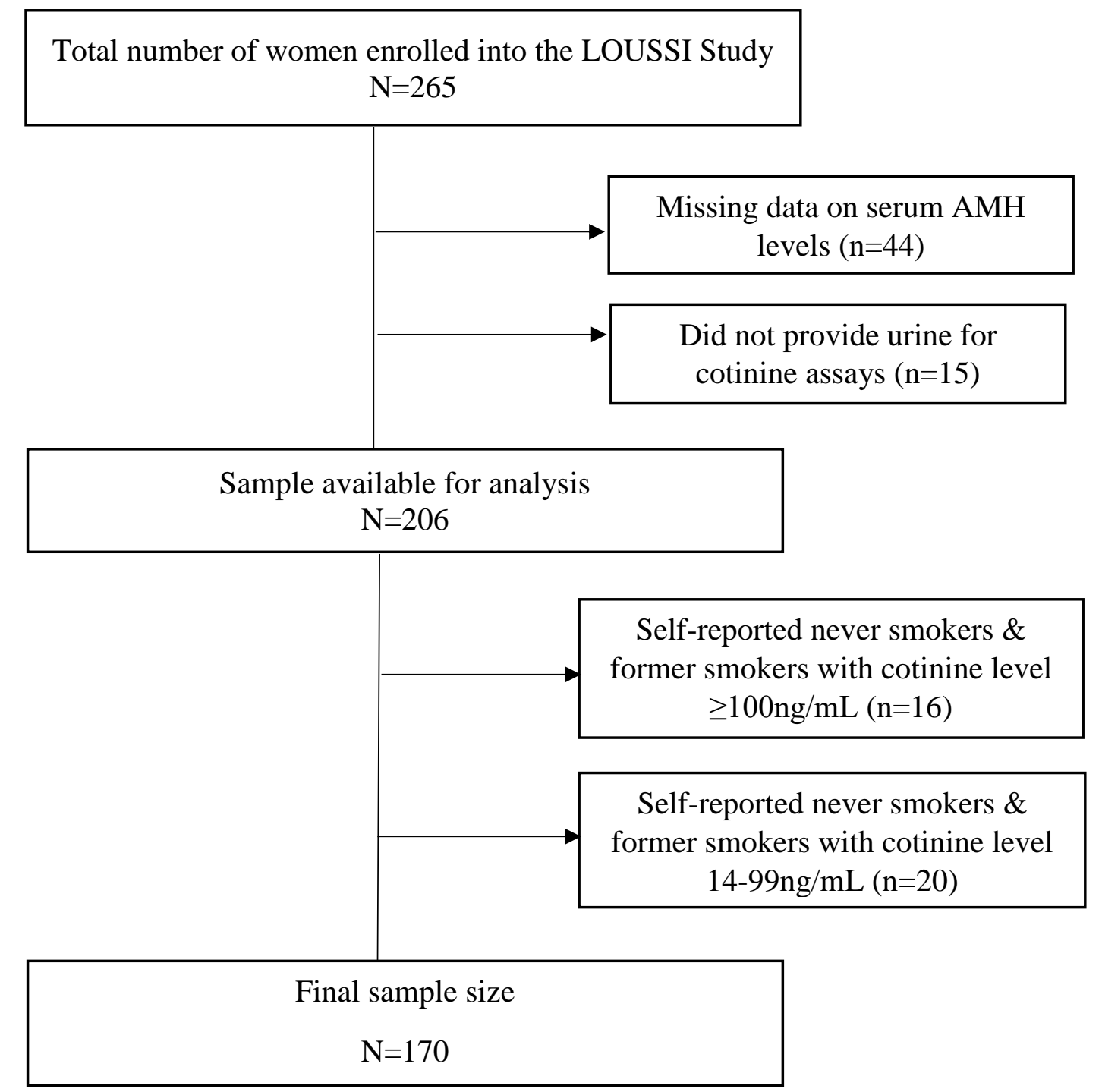


Table 5. 1. Characteristics of Women in the Study Stratified by Smoking Status (N =170)

\begin{tabular}{|c|c|c|c|}
\hline & $\begin{array}{c}\text { Nonsmokers } \\
\mathbf{N}(\%) \\
(\mathbf{n}=\mathbf{1 3 9}) \\
\end{array}$ & $\begin{array}{c}\text { Smokers } \\
\mathbf{N}(\%) \\
(\mathbf{n}=31) \\
\end{array}$ & P-value \\
\hline Age (years) & & & 0.04 \\
\hline $21-25$ & $16(11.5)$ & $9(30.0)$ & \\
\hline $26-30$ & $26(18.7)$ & $7(23.3)$ & \\
\hline $31-35$ & $50(36.0)$ & $8(26.7)$ & \\
\hline $36-45$ & $47(33.8)$ & $6(20.0)$ & \\
\hline Race & & & 0.01 \\
\hline White & $73(52.9)$ & $17(54.9)$ & \\
\hline Black & $32(23.2)$ & $13(41.9)$ & \\
\hline Other & $33(23.9)$ & $1(3.2)$ & \\
\hline Age at menarche(years) mean (s.d) & $12.5(1.9)$ & $11.9(1.5)$ & 0.10 \\
\hline Body Mass Index & & & 0.17 \\
\hline $17.5-24.9$ & $36(26.3)$ & $8(25.8)$ & \\
\hline $25-29.9$ & $44(32.1)$ & $6(19.4)$ & \\
\hline $30-34.9$ & $25(18.2)$ & $11(35.4)$ & \\
\hline$\geq 35$ & $32(23.4)$ & $6(19.4)$ & \\
\hline Poly Cystic Ovary Syndrome & & & 0.80 \\
\hline No & $83(60.6)$ & $18(58.1)$ & \\
\hline Yes & $54(39.4)$ & $13(41.9))$ & \\
\hline \multicolumn{4}{|l|}{ Parity } \\
\hline None & $60(43.8)$ & $9(29.0)$ & 0.30 \\
\hline One & $31(22.6)$ & $8(25.8)$ & \\
\hline Two or more & $46(33.6)$ & $14(45.2)$ & \\
\hline Marital Status & & & $<0.001$ \\
\hline Single & $31(23.7)$ & $20(71.4)$ & \\
\hline Married & $100(76.3)$ & $8(28.6)$ & \\
\hline $\begin{array}{l}\text { Serum AMH }(\mathrm{ng} / \mathrm{mL}) \text { median } \\
\text { (interquartile range) }\end{array}$ & $2.33(4.0)$ & $2.07(2.0)$ & 0.48 \\
\hline Diminished ovarian reserve (DOR) & & & 0.59 \\
\hline No & $101(72.7)$ & $24(77.4)$ & \\
\hline Yes & $38(27.3)$ & $7(22.6)$ & \\
\hline $\begin{array}{l}\text { Antral Follicle Count (AFC) } \\
\text { median (interquartile range) }\end{array}$ & $21(17)$ & $17(11)$ & 0.48 \\
\hline
\end{tabular}


Table 5. 2. NAT2 Phenotype of Women in the Study $(\mathrm{N}=156)$

\begin{tabular}{|c|c|c|c|}
\hline & $\begin{array}{c}\text { Rapid/ Intermediate Acetylators } \\
\begin{array}{c}\mathbf{N}(\%) \\
(\mathbf{n}=95)\end{array}\end{array}$ & $\begin{array}{c}\text { Slow Acetylators } \\
\mathbf{N}(\%) \\
(\mathbf{n}=61)\end{array}$ & P-value \\
\hline Smoking Status & & & 0.91 \\
\hline Smokers & $18(19.0)$ & $12(19.7)$ & \\
\hline Nonsmokers & 77 (81.0) & $49(80.3)$ & \\
\hline Race & & & 0.79 \\
\hline White & $51(54.2)$ & $31(50.8)$ & \\
\hline Black & $23(24.5)$ & $18(29.5)$ & \\
\hline Other & $20(21.3)$ & $12(19.7)$ & \\
\hline
\end{tabular}

\section{Specific Aim 1 Results}

1a) Assess the association of current active smoking with ovarian reserve.

1b) Determine a dose-response relationship between current active smoking (as measured by number of cigarettes smoked per day) and ovarian reserve.

The association of current active smoking with ovarian reserve was assessed using linear regression with $\ln (\mathrm{AMH})$ as the outcome and logistic regression for DOR. Results from the univariate analyses are presented in Table 5.3. No statistically significant association was observed between current active smoking and ovarian reserve (AMH and DOR) (Table 5.3). Age and PCOS status were significantly associated with both serum AMH level and DOR. A 50\% (95\% CI: -72.0 - -9.8) decrease in AMH level was reported among women between ages 31 to 35 years and a 64\% (95\% CI: -79.9 - 35.2) decrease among women older than 35 years of age (Table 5.3). A similar inverse association was found between age and DOR, with women between the ages of 36 to 45 years having 6 times the odds of DOR (OR=5.89; 95\% CI:1.63 - 21.38) compared to women 21 to 25 years of age (Table 5.3). A PCOS diagnosis was associated with a significant increase in AMH levels and a decrease in the odds of DOR (Table 5.3). 
Neither serum AMH levels nor DOR was found to be significantly associated with race, BMI, age at menarche or parity (Table 5.3). However, BMI category and parity were considered for inclusion in all multivariable models with $\ln (\mathrm{AMH})$ and DOR as outcomes because they were related to ovarian reserve at a significance level of $\mathrm{p}<0.20$. All regression models were adjusted for age and PCOS status.

Adjusted linear regression models for the association of current active smoking and percentage change in AMH are presented in Table 5.4. There was no significant association found between current active smoking or the number of cigarette smoked and AMH level (smoker vs. nonsmoker: \% $\Delta$ in AMH -5.6; 95\% CI: -48.2 - 72.1) (Table 5.4).

After adjusting for age and PCOS status, current active smoking was not associated with DOR $(\mathrm{OR}=1.14 ; 95 \% \mathrm{CI}: 0.41-3.18)$. No dose-response relationship was found between number of cigarette smoked and DOR, regardless of whether the exposure was measured as continuous $(\mathrm{OR}=1.04$; $95 \% \mathrm{CI}: 0.98-1.10, \mathrm{p}=0.19)$ or categorized $(1-9$ cigarettes /day: $\mathrm{OR}=0.30 ; 95 \% \mathrm{CI}: 0.03-2.6) ; \geq 10$ cigarettes /day: $\mathrm{OR}=2.02$; 95\% CI: $0.62-6.69$ ) (Table 5.4). When analyses were limited to women without PCOS, the effect of current smoking on DOR was stronger but did not reach statistical significance $(\mathrm{OR}=1.75 ; 95 \% \mathrm{CI}: 0.49-6.33)$ (Table 5.5). 
Table 5. 3. Univariate Analyses for the Association of Current Active Smoking with Percent Change in Anti -Müllerian hormone (AMH) and Risk of Diminished Ovarian Reserve (DOR)

\begin{tabular}{|c|c|c|}
\hline & $\begin{array}{c}\text { \% Change in AMH } \\
(95 \% \text { CI })\end{array}$ & OR $(95 \% \mathrm{CI})$ for $\mathrm{DOR}$ \\
\hline \multicolumn{3}{|l|}{ Smoking Status } \\
\hline Nonsmoker & Referent & 1.00 (Referent) \\
\hline Smoker & $-5.6(-48.2-72.1)$ & $0.78(0.31-1.95)$ \\
\hline p-value & 0.85 & 0.59 \\
\hline \multicolumn{3}{|l|}{ Age (years) } \\
\hline $21-25$ & Referent & $1.00(\text { Referent })^{\mathrm{a}}$ \\
\hline $26-30$ & $20.5(-36.3-127.9)$ & $0.48(0.08-3.08)$ \\
\hline $31-35$ & $-49.7(-72.0--9.8)$ & $3.63(0.98-13.39)$ \\
\hline $36-45$ & $-63.9(-79.9--35.2)$ & $5.89(1.63-21.38)$ \\
\hline p-value & $<0.001$ & 0.0012 \\
\hline \multicolumn{3}{|l|}{ Race } \\
\hline White & Referent & 1.00 (Referent) \\
\hline Black & $11.0(-29.5-75.0)$ & $0.73(0.34-1.56)$ \\
\hline Other & $-23.6(-55.6-31.6)$ & $0.98(0.41-2.33)$ \\
\hline$p$-value & 0.46 & 0.69 \\
\hline Age at menarche & $8.8(-2.6-21.6)$ & $0.89(0.74-1.07)$ \\
\hline$p$-value & 0.14 & 0.22 \\
\hline \multicolumn{3}{|l|}{ Body Mass Index } \\
\hline $17.5-24.9$ & Referent & $1.00{\text { (Referent })^{\mathrm{b}}}$ \\
\hline $25-29.9$ & $-17.2(-51.8-42.3)$ & $1.64(0.63-4.28)$ \\
\hline $30-34.9$ & $-35.0(-63.3-16.1)$ & $1.94(0.71-5.28)$ \\
\hline$\geq 35$ & $-13.0(-51.1-54.9)$ & $2.33(0.88-6.21)$ \\
\hline$p$-value & 0.53 & 0.38 \\
\hline \multicolumn{3}{|l|}{ PCOS } \\
\hline $\mathrm{No}$ & Referent & 1.00 (Referent) \\
\hline Yes & $173.5(86.1-301.9)$ & $0.32(0.15-0.69)$ \\
\hline p-value & $<0.001$ & 0.004 \\
\hline \multicolumn{3}{|l|}{ Parity } \\
\hline None & Referent & $1.00(\text { Referent })^{\mathrm{c}}$ \\
\hline One & $-27.3(-55.8-19.8)$ & $1.45(0.63-3.34)$ \\
\hline Two or more & $-39.1(-61.1--4.7)$ & $1.25(0.58-2.70)$ \\
\hline p-value & 0.09 & 0.67 \\
\hline
\end{tabular}


Table 5. 4. Multivariable Model for Association of Current Active Smoking with Percent Change in Anti -Müllerian hormone (AMH) and Risk of Diminished Ovarian Reserve (DOR) (N=167)

\begin{tabular}{|c|c|c|}
\hline & $\begin{array}{c}\text { \% Change in AMH } \\
(95 \% \text { CI })\end{array}$ & OR $(95 \% \mathrm{CI})$ for $\mathrm{DOR}$ \\
\hline \multicolumn{3}{|l|}{ Model 1a } \\
\hline \multicolumn{3}{|l|}{ Current smoking status } \\
\hline Nonsmoker, $n=137$ & Referent & 1.00 (Referent) \\
\hline Smoker, $n=30$ & $-24.7(-57.1-32.1)$ & $1.14(0.41-3.18)$ \\
\hline$p$-value & 0.32 & 0.81 \\
\hline \multicolumn{3}{|l|}{ Model 1b } \\
\hline $\begin{array}{l}\text { Number of cigarettes/day } \\
\text { p-value }\end{array}$ & $\begin{array}{c}-1.95(-5.10-1.30) \\
0.23\end{array}$ & $\begin{array}{c}1.04(0.98-1.10) \\
0.19\end{array}$ \\
\hline \multicolumn{3}{|c|}{ Model 1bii } \\
\hline \multicolumn{3}{|c|}{ Number of cigarettes/day (categories) } \\
\hline $0, n=137$ & Referent & 1.00 (Referent) \\
\hline $1-9, n=12$ & $-9.17(-60.59-109.29)$ & $0.30(0.03-2.63)$ \\
\hline$\geq 10, n=18$ & $-33.42(-210-33.19)$ & $2.02(0.62-6.69)$ \\
\hline p-value & 0.51 & 0.25 \\
\hline
\end{tabular}

Adjusted for age and polycystic ovary syndrome

Table 5. 5. Multivariable Model for Association of Current Active Smoking with Percent Change in Anti -Müllerian hormone (AMH) and Risk of Diminished Ovarian Reserve (DOR) Among Women Without Polycystic Ovary Syndrome (PCOS) $(\mathbf{N}=100)$

\begin{tabular}{lcc}
\hline & $\begin{array}{l}\text { \% Change in AMH } \\
(\mathbf{9 5 \%} \mathbf{C I})\end{array}$ & OR (95\% CI) for DOR \\
\hline Model 1a & & \\
Current smoking status & Referent & 1.00 (Referent) \\
$\quad$ Nonsmoker, $n=83$ & $-10.3(-59.94-100.92)$ & $1.75(0.49-6.33)$ \\
$\quad$ Smoker, $n=17$ & 0.79 & 0.39 \\
$p$-value & & $1.06(0.99-1.14)$ \\
Model 1b & $-0.75(-4.89-3.58)$ & 0.08 \\
Number of cigarettes/day & 0.73 & \\
$\quad p$-value & & $1.00($ Referent $)$ \\
Model 1bii & Referent & $0.49(0.05-5.06)$ \\
Number of cigarettes/day & $-17.69(-74.59-166.61)$ & 0.25 \\
$\quad 0, n=83$ & $-4.76(-65.04-159.46)$ & $3.44(0.68-17.31)$ \\
$1-9, n=7$ & 0.95 & 0.25 \\
$\geq 10, n=10$ & & \\
$p$-value & &
\end{tabular}


Specific Aim 1c- Assess a dose-response relationship between cumulative lifetime smoking (as measured by pack-years reported on the questionnaire) and ovarian reserve.

Overall $n=204$ women were included in the analysis for Specific Aim 1c.

Cumulative lifetime of smoking was assessed for both smokers and former smokers via questionnaire. Cumulative lifetime of smoking was not significantly associated with change in serum AMH levels or increased risk of DOR (Table 5.6). The association remained insignificant when the analysis was restricted to women without PCOS (Table 5.7).

Table 5. 6. Association of Cumulative Lifetime Smoking Exposure as a Continuous Measure and as a Categorical Measure with Percent Change in AMH and Risk of $\operatorname{DOR}(\mathbf{N}=204)$

\begin{tabular}{lcc}
\hline & $\begin{array}{l}\text { \% Change in AMH } \\
(\mathbf{9 5 \%} \mathbf{C I})\end{array}$ & OR (95\% CI) for DOR \\
\hline $\begin{array}{l}\text { Pack-years, Continuous } \\
\text { Lifetime exposure (pack-years) } \\
\text { p-value }\end{array}$ & $-1.46(-5.70-2.98)$ & $1.05(0.97-1.13)$ \\
$\begin{array}{l}\text { Pack-years, Categorical } \\
\text { Never Smoker, } n=149\end{array}$ & 0.51 & 0.24 \\
$1-5, n=35$ & Referent & $1.00($ Referent $)$ \\
$>5, n=20$ & $9.64(-33.28-80.15)$ & $0.65(0.24-1.77)$ \\
$p$-value & $-27.62(-61.36-35.58)$ & $1.68(0.54-5.17)$ \\
\hline Adjusted for age and polycystic ovary syndrome & 0.52 & 0.41 \\
\hline
\end{tabular}


Table 5. 7. Association of Cumulative Lifetime Smoking Exposure as a Continuous Measure and as a Categorical Measure with Percent Change in AMH and Risk of DOR Among Women Without Polycystic Ovary Syndrome (N=123)

\begin{tabular}{|c|c|c|}
\hline & $\begin{array}{l}\text { \% Change in AMH } \\
(95 \% \text { CI })\end{array}$ & OR $(95 \% \mathrm{CI})$ for $\mathrm{DOR}$ \\
\hline \multicolumn{3}{|l|}{ Pack-years, Continuous } \\
\hline Lifetime exposure (pack-years) & $-1.20(-6.37-4.26)$ & $1.08(0.99-1.18)$ \\
\hline$p$-value & 0.66 & 0.08 \\
\hline \multicolumn{3}{|l|}{ Pack-years, Categorical } \\
\hline Never Smoker, $n=92$ & Referent & 1.00 (Referent) \\
\hline $1-5, n=21$ & $-12.78(-55.95-72.67)$ & $1.04(0.34-3.18)$ \\
\hline$>5, n=10$ & $-7.20(-63.52-136.06)$ & $3.16(0.68-14.12)$ \\
\hline$p$-value & 0.92 & 0.32 \\
\hline
\end{tabular}

Specific Aim 2- Determine if the association of current active smoking with ovarian reserve differ by race, comparing African-American with Caucasians (NHW) women.

The interaction between race and current active smoking on ovarian reserve was assessed using logistic regression, with DOR as the main outcome. Results of the analysis are presented in Table 5.8. After adjusting for age and PCOS diagnosis, no significant interaction was found between current active smoking and race on DOR ( $p$-value $=0.91$ ). Results were non-significant for women without PCOS (Table 5.9). Similarly, no interaction was found between current active smoking and race on AMH levels (data not shown). 
Table 5. 8. Interaction of Current Active Smoking and Race on Risk of Diminished Ovarian Reserve (DOR) $(\mathrm{N}=135)$

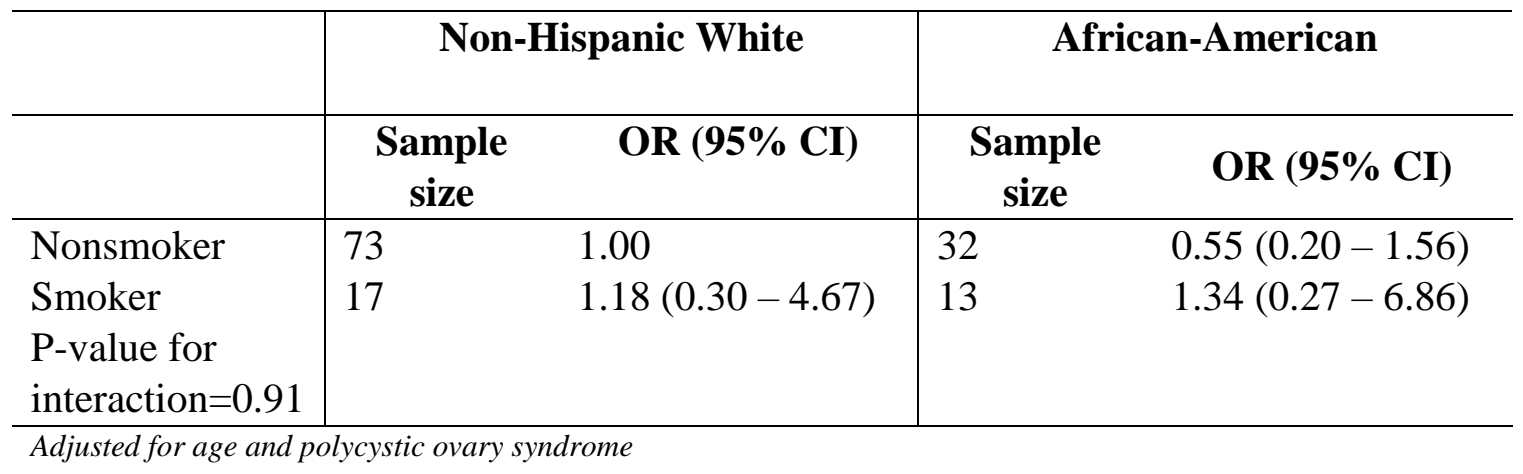

Table 5. 9. Interaction of Current Active Smoking and Race on Risk of Diminished Ovarian Reserve (DOR) Among Women without Polycystic Ovary Syndrome $(\mathrm{N}=82)$

\begin{tabular}{|c|c|c|c|c|}
\hline & \multicolumn{2}{|c|}{ Non-Hispanic White } & \multicolumn{2}{|c|}{ African-American } \\
\hline & $\begin{array}{c}\text { Sample } \\
\text { size }\end{array}$ & OR $(95 \% \mathrm{CI})$ & $\begin{array}{c}\text { Sample } \\
\text { size }\end{array}$ & OR $(95 \% \mathrm{CI})$ \\
\hline $\begin{array}{l}\text { Nonsmoker } \\
\text { Smoker } \\
\text { P-value for } \\
\text { interaction }=0.41\end{array}$ & $\begin{array}{l}41 \\
10\end{array}$ & $\begin{array}{l}1.00 \\
1.25(0.23-6.82)\end{array}$ & $\begin{array}{l}23 \\
8\end{array}$ & $\begin{array}{l}0.48(0.14-1.64) \\
3.74(0.50-27.79)\end{array}$ \\
\hline
\end{tabular}

Specific Aim 3- Determine interaction between current active smoking and NAT2 acetylator status on ovarian reserve.

The effect measure modification by NAT2 was assessed using logistic regression with DOR as outcome. After adjusting for age, race and PCOS status, there was no significant interaction with NAT2 (Table 5.10); among slow acetylators: the association between smoking and DOR was OR=1.63 (95\% CI:0.27-9.94); whereas in rapid acetylators, $\mathrm{OR}=0.89(95 \% \mathrm{CI}: 0.25-3.24)$. 
When analysis was limited to women without PCOS, the effect modification by NAT2 acetylator status became stronger but did not reach statistical significance (Table 5.11). Current active smokers who are slow acetylators had twice the odds of DOR $(\mathrm{OR}=2.51 ; 95 \%$ CI: $0.15-42.64)($ Table 5.11).

Table 5. 10. Interaction of Current Active Smoking and NAT2 Acetylator Phenotype on Risk of Diminished Ovarian Reserve (DOR) $(\mathrm{N}=156)$

\begin{tabular}{|c|c|c|c|c|}
\hline & \multicolumn{2}{|c|}{ Rapid/Intermediate } & \multicolumn{2}{|r|}{ Slow } \\
\hline & $\begin{array}{c}\text { Sample } \\
\text { size }\end{array}$ & OR (95\% CI) & $\begin{array}{c}\text { Sample } \\
\text { size }\end{array}$ & OR $(95 \% \mathrm{CI})$ \\
\hline $\begin{array}{l}\text { Nonsmoker } \\
\text { Smoker } \\
\text { P-value for } \\
\text { interaction=0.29 }\end{array}$ & $\begin{array}{l}77 \\
18\end{array}$ & $\begin{array}{l}1.00 \\
0.89(0.25-3.24)\end{array}$ & $\begin{array}{l}49 \\
12\end{array}$ & $\begin{array}{l}0.65(0.26-1.62) \\
1.63(0.27-9.94)\end{array}$ \\
\hline
\end{tabular}

Table 5. 11. Interaction of Current Active Smoking and NAT2 Acetylator Phenotype on Risk of Diminished Ovarian Reserve (DOR) Among Women Without Polycystic Ovary Syndrome $(\mathrm{N}=91)$

\begin{tabular}{|c|c|c|c|c|}
\hline & \multicolumn{2}{|c|}{ Rapid/Intermediate } & \multicolumn{2}{|r|}{ Slow } \\
\hline & $\begin{array}{c}\text { Sample } \\
\text { size }\end{array}$ & OR (95\% CI) & $\begin{array}{c}\text { Sample } \\
\text { size }\end{array}$ & OR $(95 \%$ CI $)$ \\
\hline $\begin{array}{l}\text { Nonsmoker } \\
\text { Smoker } \\
\text { P-value for } \\
\text { interaction }=0.23\end{array}$ & $\begin{array}{l}49 \\
13\end{array}$ & $\begin{array}{l}1.00 \\
1.15(0.28-4.76)\end{array}$ & $\begin{array}{l}25 \\
4\end{array}$ & $\begin{array}{l}0.37(0.11-1.14) \\
2.51(0.15-42.64)\end{array}$ \\
\hline
\end{tabular}




\section{F. DISCUSSION}

This study assessed the association of smoking with ovarian reserve among women of reproductive age. The existing evidence on smoking and ovarian reserve as

measured by AMH has been inconclusive [12, 16, 17, 20, 21, 55, 93-98]. This is the first study to explore effect modification by race and NAT2 phenotype as a possible cause of inconsistency in previous studies, and the first study of smoking and ovarian reserve to validate self-reported exposure status with cotinine.

The findings from this cross-sectional analysis are consistent with previous prospective [55], retrospective [96] and cross-sectional studies [12, 97, 98] that found no significant association between smoking and ovarian reserve. However, other studies did find a significant association between smoking and ovarian reserve [16, $17,20,21,93-95]$. If an association truly exists, then nonsignificant results from previous studies may be due to a crude assessment of smoking exposure status based on self-report and not accounting for duration and quantity of smoking. Further, previous results are difficult to reconcile because of differences in the definition of exposure status and adjustment factors across studies. 
Only a few studies have explored a dose-response relationship between smoking and ovarian reserve $[22,59,95,96]$. This study provided a detailed assessment of smoking status, validated current exposure status with cotinine, assessed a dose-response relationship, and estimated cumulative lifetime exposure using the quantity and duration of smoking. While effect sizes did not reach statistical significance, the magnitude and direction of associations suggest that smoking may reduce ovarian reserve especially among heavy smokers. White et al. (2016) in a cross-sectional study of 913 premenopausal women, found a significant association between self-reported smoking and serum AMH among women who smoked 20 or more cigarettes /day $(-56.2 \%$, 95\%CI: $-80.3,-2.8 \%$ ) [95]. Similar findings with number of pack-years smoked were reported by Dolleman et al. where significant association between smoking and agespecific serum AMH was found for 10-15 pack-years $(\beta=-7.0, p=0.003)$ and 15-20 packyears $(\beta=-8.5, \mathrm{p}=0.001)$ pack-years but not for 5-10 pack-years $(\beta=-3.1, \mathrm{p}=0.15)[59]$.

Other studies that reported significant inverse associations between smoking and ovarian reserve (as measured by $\mathrm{AMH}$ ) reported moderate $[16,94,95]$ to high $[17,20$, 21,93 ] prevalence of smoking in the study population. Considering the established relationship between smoking and early age at menopause [142-146], it is plausible that 
smoking is associated with ovarian aging and reserve, but the association was not detected in this study due to low frequency of heavy smokers.

Studies on the association of smoking with ovarian reserve varied in the covariates selected for adjustment. None of the studies controlled for PCOS, even though several of the studies $[17,20-22,55,93]$ were conducted among infertile or sub-fertile populations. Women with PCOS diagnosis have been shown to have significantly elevated AMH levels $[63,64]$. In analyses that excluded women with PCOS diagnosis, effect sizes were generally stronger, suggesting PCOS is not only a confounder, but also an effect modifier of the association of smoking with ovarian reserve. Therefore, controlling for and stratifying on PCOS was a major improvement in the study design compared to prior studies.

Timing of exposure may be a very important consideration for environmental predictors of $\mathrm{AMH}$. For example, exposure to toxins during early development may be more influential on ovarian reserve than exposure as an adult. Experimental studies in animals shows that smoking inhibits follicular development; causes premature luteinization and atresia of the preovulatory follicle; reduces oocyte vascularization and maturation; impairs steroidogenesis; and increases chromosomal errors; and cytotoxicity; in a stage dependent manner [75-78]. Most studies on the association of smoking with ovarian reserve including this study, did not account for the timing of exposure.

\section{Effect Modification by Race}

Racial disparity in the association of smoking with ovarian reserve is largely unknown. The results of this study suggest that the risk of diminished ovarian reserve may be increased in African-American smokers. Schuh-Huerta et al. (2011) in a 
genome-wide association (GWA) analyses on hormonal markers of ovarian reserve (FSH and AMH) identified genome-wide significant SNPs associated with FSH (rs6543833, rs12465811, rs11255291) and AMH levels (rs9875589) in an AfricanAmerican population [16]. One SNP (rs12213875) identified in Caucasians was associated with a mean decrease of $1.3+1.4 \mathrm{pM}$ in AMH among GG genotypes compared to an increase of $13.8+4.2 \mathrm{pM}$ and $9.9+2.1 \mathrm{pM}$ in the $\mathrm{AA}$ and $\mathrm{AG}$ genotypes, respectively [16]. While these results provide evidence for underlying genetic variations in ovarian reserve by race, disparities in environmental and behavioral factors such as smoking, also need to be explored.

\section{Effect Modification by NAT2}

This study is the first to explore population differences in NAT2 genotype frequencies as a possible reason for the inconsistency in the association of smoking with ovarian reserve. Results from this study, though not statistically significant, suggests that the risk of diminished ovarian reserve may be increased for current smokers who are slow NAT2 acetylators. Polymorphisms in the NAT2 gene modify susceptibility to harmful heterocyclic and aromatic amines constituents in tobacco smoke [25, 26, 100, 101]. High levels of DNA adduct of 4-aminobiphenyl, a carcinogenic aromatic amine in tobacco smoke, have been reported in tissues of smokers [103, 104, 106-112, 147]. Additionally, it has been shown that slow NAT2 acetylators have variable reductions in catalytic activities that make them more susceptible to the effect of toxins [26, 100, 101, 114]. Given the significantly reduced fecundability reported among current smokers who are slow acetylators by Taylor et al. (2011), it is plausible that the effect is modulated through a reduction in ovarian 
reserve. As reviewed earlier in this dissertation [29, 114, 117], slow acetylator NAT2 phenotypes result from different mechanisms based on genotype, suggesting that the slow phenotype is not homogenous. Therefore, treating all slow acetylators as a single group may be diluting any effects present in a particular haplotype. Genetic heterogeneity of the slow NAT2 phenotype was investigated by assessing the interaction of smoking with individual NAT2 alleles and genotypes on ovarian reserve but analyses was limited by small sample size (data not shown). More studies, with a large sample size, are needed to clarify the role of NAT2 genotypes in the association of smoking with ovarian reserve.

\section{Strengths and Limitations}

Strengths of this study included the novel investigation of the impact of race and NAT2 acetylator status on the relationship of smoking with ovarian reserve in a diverse population of reproductive-age women. Comprehensive questionnaires permitted detailed characterization of exposure. Validation of exposure status with cotinine helped reduce misclassification error that may have occurred with use of self-report alone. Another strength of this study is with the use of an objective, a sensitive and reliable marker of ovarian reserve, AMH [58, 148, 149]. Studies show a high correlation between $\mathrm{AFC}$ and $\mathrm{AMH}[55,56]$. In addition, assessing information on outcome measures and covariates from medical records reduced the likelihood of differential information collection among smokers and nonsmokers.

This study has several limitations which are peculiar to most cross-sectional study designs. Given the non-directionality and retrospective nature of the study 
design, temporality cannot be established impacting the ability to infer a causal relationship between smoking and ovarian reserve.

Recruiting participants from a single clinic may result in lack of generalizability. The study population may be significantly different from the general population of women in the U.S. limiting the generalizability of results of the study to populations not seeking fertility care.

Another potential source of bias is missing information. Women excluded from the study for missing information on important variables may be different in significant ways from women who had complete data. For example, only $78 \%$ of all women who participated in the LOUSSI study had AMH levels measured. If some women declined a fertility workup, and those women were different from the women who agreed to do the work up with regard to their smoking status and AMH levels, selection bias could result. Based on the cost associated with pursuing fertility treatment $[3,4]$, it may be the case that women excluded are of low socioeconomic class, potentially compromising both internal and external validity.

Recall bias is another potential limitation of this study. Although current smoking status was validated with cotinine, the dose and duration of exposure was largely based on self-report with the possibility of under-reporting (social desirability bias) or simply misremembering. Smokers and former smokers under-reporting their exposure status may bias effect estimate towards the null. While access to medical records of participants provided access to important information relating to smoking and ovarian reserve, data on some important covariates were missing in some participants (alcohol use missing in 65\%). For example, although the associations of 
socioeconomic status and oral contraceptive use on ovarian reserve have not been established, it is uncertain how these unmeasured covariates may influence effect estimates obtained from this study.

Finally, this study was limited by the small sample of smokers in the study population which reduced the power to detect the main effect of smoking or its' potential interaction with race and NAT2 on ovarian reserve. Consequently, it cannot be certain whether the null associations are attributable to an actual lack of association or to inadequate statistical power. Future research should expand recruitment to other gynecological and fertility clinics and/or consider a meta-analysis to increase sample size.

\section{Conclusion}

Neither current cigarette smoking nor cumulative lifetime exposure to cigarette smoking was significantly associated with ovarian reserve in this study, although the directions of effect were consistent with the notion that smoking accelerates ovarian aging and follicle atresia. Larger and more representative studies are needed to better understand the relationship between smoking and ovarian reserve, especially for populations attempting pregnancy. As this study has set a precedent, future studies in this area should attempt to assess lifetime exposure to toxins from cigarette smoke with attention to dose and to particular developmental windows and should validate current smoking status with cotinine.

Additional data is necessary to better define the role of race and NAT2 polymorphisms with diminished ovarian reserve in women of reproductive-age following exposure to toxins such as those found in cigarette smoke. 


\section{REFERENCES}

1. Chandra, A., C.E. Copen, and E.H. Stephen, Infertility and impaired fecundity in the United States, 1982-2010: data from the National Survey of Family Growth. Natl Health Stat Report, 2013(67): p. 1-18, 1 p following 19.

2. Chandra, A., C.E. Copen, and E.H. Stephen, Infertility service use in the United States: data from the National Survey of Family Growth, 1982-2010. Natl Health Stat Report, 2014(73): p. 1-21.

3. Katz, P., et al., Costs of infertility treatment: results from an 18-month prospective cohort study. Fertil Steril, 2011. 95(3): p. 915-21.

4. Collins, J.A., et al., An estimate of the cost of in vitro fertilization services in the United States in 1995. Fertil Steril, 1995. 64(3): p. 538-45.

5. Ramezanzadeh, F., et al., Surveying of relationship between anxiety, depression and duration of infertility. International Congress Series, 2004. 1271: p. 334-337.

6. Karimzadeh, M., et al., Psychological Disorders among Iranian Infertile Couples Undergoing Assisted Reproductive Technology (ART). Iran J Public Health, 2017. 46(3): p. 333-341. 
7. Gelbaya, T.A., et al., Definition and epidemiology of unexplained infertility. Obstet Gynecol Surv, 2014. 69(2): p. 109-15.

8. Brugo-Olmedo, S., C. Chillik, and S. Kopelman, Definition and causes of infertility. Reprod Biomed Online, 2001. 2(1): p. 41-53.

9. Center for Disease Control and Prevention, American Society for Reproductive Medicine, and Society for Assisted Reproductive Technology, 2012 Assisted Reproductive Technology National Summary Report., U.D.o.H.a.H. Services, Editor. 2014.

10. Practice Committee of the American Society for Reproductive, M., Testing and interpreting measures of ovarian reserve: a committee opinion. Fertil Steril, 2015. 103(3): p. e9-e17.

11. Practice Committee of the American Society for Reproductive, M., Diagnostic evaluation of the infertile female: a committee opinion. Fertil Steril, 2015. 103(6): p. e44-50.

12. Jung, S., et al., Demographic, lifestyle, and other factors in relation to antimullerian hormone levels in mostly late premenopausal women. Fertil Steril, 2017. 107(4): p. 1012-1022.e2.

13. Hazout, A., et al., Serum antimullerian hormone/mullerian-inhibiting substance appears to be a more discriminatory marker of assisted reproductive technology outcome than follicle-stimulating hormone, inhibin B, or estradiol. Fertil Steril, 2004. 82(5): p. 1323-9. 
14. Seifer, D.B. and D.T. Maclaughlin, Mullerian Inhibiting Substance is an ovarian growth factor of emerging clinical significance. Fertil Steril, 2007. 88(3): p. 53946.

15. Broer, S.L., et al., The role of antimullerian hormone in prediction of outcome after IVF: comparison with the antral follicle count. Fertil Steril, 2009. 91(3): p. 705-14.

16. Schuh-Huerta, S.M., et al., Genetic variants and environmental factors associated with hormonal markers of ovarian reserve in Caucasian and African American women. Hum Reprod, 2012. 27(2): p. 594-608.

17. Freour, T., et al., Active smoking compromises IVF outcome and affects ovarian reserve. Reprod Biomed Online, 2008. 16(1): p. 96-102.

18. El-Nemr, A., et al., Effect of smoking on ovarian reserve and ovarian stimulation in in-vitro fertilization and embryo transfer. Hum Reprod, 1998. 13(8): p. 2192-8.

19. Radin, R.G., et al., Active and passive smoking and fecundability in Danish pregnancy planners. Fertil Steril, 2014. 102(1): p. 183-191.e2.

20. Fuentes, A., et al., Decreased anti-Mullerian hormone concentration in follicular fluid of female smokers undergoing artificial reproductive techniques. Chemosphere, 2012. 88(4): p. 403-6.

21. Freour, T., et al., Ovarian reserve and in vitro fertilization cycles outcome according to women smoking status and stimulation regimen. Arch Gynecol Obstet, 2012. 285(4): p. 1177-82.

22. Caserta, D., et al., The influence of cigarette smoking on a population of infertile men and women. Arch Gynecol Obstet, 2013. 287(4): p. 813-8. 
23. Dubinskaia, E.D., et al., [Role of the genetic factors, detoxication systems and oxidative stress in the pathogenesis of endometriosis and infertility (review)]. Vestn Ross Akad Med Nauk, 2013(8): p. 14-9.

24. Taylor, K.C., et al., Alcohol, smoking, and caffeine in relation to fecundability, with effect modification by NAT2. Ann Epidemiol, 2011. 21(11): p. 864-72.

25. Weber, W.W. and D.W. Hein, N-acetylation pharmacogenetics. Pharmacol Rev, 1985. 37(1): p. 25-79.

26. Walker, K., et al., Genetic polymorphism in N-Acetyltransferase (NAT): Population distribution of NAT1 and NAT2 activity. J Toxicol Environ Health B Crit Rev, 2009. 12(5-6): p. 440-72.

27. Wu, H., et al., Association Between N-acetyltransferase 2 Polymorphism and Bladder Cancer Risk: Results From Studies of the Past Decade and a MetaAnalysis. Clin Genitourin Cancer, 2016. 14(2): p. 122-9.

28. Costa, S., et al., A slow acetylator genotype associated with an increased risk of advanced cervical cancer. J Cancer Res Clin Oncol, 2002. 128(12): p. 678-82.

29. Hein, D.W., N-acetyltransferase 2 genetic polymorphism: effects of carcinogen and haplotype on urinary bladder cancer risk. Oncogene, 2006. 25(11): p. 164958.

30. World Health Organization. Sexual and Reproductive Health. Infertility definitions and Terminology. [cited 2017 June 22]; Available from: http://www.who.int/reproductivehealth/topics/infertility/definitions/en/. 
31. Practice Committee of the American Society for Reproductive Medicine, Diagnostic evaluation of the infertile male: a committee opinion. Fertil Steril, 2015. 103(3): p. e18-25.

32. Lashen, H., Investigations for infertility. Obstetrics, Gynaecology \& Reproductive Medicine, 2007. 17(7): p. 211-216.

33. Mascarenhas, M.N., et al., National, regional, and global trends in infertility prevalence since 1990: a systematic analysis of 277 health surveys. PLoS Med, 2012. 9(12): p. e1001356.

34. Centers for Disease Control and Prevention and National Center for Health Statistics. National Survey of Family Growth: Key Statistics from the National Survey of Family and Growth-I Listing. [cited 2017 July 25]; Available from: https://www.cdc.gov/nchs/nsfg/key_statistics/i.htm\#infertility

35. Smith, J.F., et al., Socioeconomic disparities in the use and success of fertility treatments: analysis of data from a prospective cohort in the United States. Fertil Steril, 2011. 96(1): p. 95-101.

36. Bitler, M.P. and L. Schmidt, Utilization of infertility treatments: the effects of insurance mandates. Demography, 2012. 49(1): p. 125-49.

37. Collins, J., Cost-effectiveness of in vitro fertilization. Semin Reprod Med, 2001. 19(3): p. 279-89.

38. Chambers, G.M., et al., The economic impact of assisted reproductive technology: a review of selected developed countries. Fertil Steril, 2009. 91(6): p. 2281-94.

39. Mosher, W.D. and W.F. Pratt, Fecundity and infertility in the United States: incidence and trends. Fertil Steril, 1991. 56(2): p. 192-3. 
40. Cohen, J., N. Chabbert-Buffet, and E. Darai, Diminished ovarian reserve, premature ovarian failure, poor ovarian responder--a plea for universal definitions. J Assist Reprod Genet, 2015. 32(12): p. 1709-12.

41. Devine, K., et al., Diminished ovarian reserve in the United States assisted reproductive technology population: diagnostic trends among 181,536 cycles from the Society for Assisted Reproductive Technology Clinic Outcomes Reporting System. Fertil Steril, 2015. 104(3): p. 612-19.e3.

42. Butts, S.F., et al., Diagnosis and treatment of diminished ovarian reserve in assisted reproductive technology cycles of women up to age 40 years: the role of insurance mandates. Fertil Steril, 2013. 99(2): p. 382-8.

43. Fleming, R., et al., Assessing ovarian response: antral follicle count versus antiMullerian hormone. Reprod Biomed Online, 2015. 31(4): p. 486-96.

44. Tal, R. and D.B. Seifer, Ovarian reserve testing: a user's guide. Am J Obstet Gynecol, 2017. 217(2): p. 129-140.

45. Jayaprakasan, K., et al., A prospective, comparative analysis of anti-Mullerian hormone, inhibin-B, and three-dimensional ultrasound determinants of ovarian reserve in the prediction of poor response to controlled ovarian stimulation. Fertil Steril, 2010. 93(3): p. 855-64.

46. Hehenkamp, W.J., et al., Anti-Mullerian hormone levels in the spontaneous menstrual cycle do not show substantial fluctuation. J Clin Endocrinol Metab, 2006. 91(10): p. 4057-63. 
47. Tsepelidis, S., et al., Stable serum levels of anti-Mullerian hormone during the menstrual cycle: a prospective study in normo-ovulatory women. Hum Reprod, 2007. 22(7): p. 1837-40.

48. $\mathrm{Xu}, \mathrm{H}$., et al., Retrospective cohort study: AMH is the best ovarian reserve markers in predicting ovarian response but has unfavorable value in predicting clinical pregnancy in GnRH antagonist protocol. Arch Gynecol Obstet, 2017. 295(3): p. 763-770.

49. Silberstein, T., et al., Mullerian inhibiting substance levels at the time of HCG administration in IVF cycles predict both ovarian reserve and embryo morphology. Hum Reprod, 2006. 21(1): p. 159-63.

50. Ebner, T., et al., Basal level of anti-Mullerian hormone is associated with oocyte quality in stimulated cycles. Hum Reprod, 2006. 21(8): p. 2022-6.

51. Muttukrishna, S., et al., Antral follicle count, anti-mullerian hormone and inhibin B: predictors of ovarian response in assisted reproductive technology? Bjog, 2005. 112(10): p. 1384-90.

52. Broer, S.L., et al., Added value of ovarian reserve testing on patient characteristics in the prediction of ovarian response and ongoing pregnancy: an individual patient data approach. Hum Reprod Update, 2013. 19(1): p. 26-36.

53. Hansen, K.R., et al., Correlation of ovarian reserve tests with histologically determined primordial follicle number. Fertility and Sterility. 95(1): p. 170-175.

54. Hendriks, D.J., et al., Antral follicle count in the prediction of poor ovarian response and pregnancy after in vitro fertilization: a meta-analysis and 
comparison with basal follicle-stimulating hormone level. Fertil Steril, 2005. 83(2): p. 291-301.

55. Nardo, L.G., et al., Anti-Mullerian hormone levels and antral follicle count in women enrolled in in vitro fertilization cycles: relationship to lifestyle factors, chronological age and reproductive history. Gynecol Endocrinol, 2007. 23(8): p. 486-93.

56. van Disseldorp, J., et al., Comparison of inter- and intra-cycle variability of antiMullerian hormone and antral follicle counts. Hum Reprod, 2010. 25(1): p. 2217.

57. Weenen, C., Anti-Mullerian hormone expression pattern in the human ovary: potential implications for initial and cyclic follicle recruitment. Molecular Human Reproduction, 2004. 10(2): p. 77-83.

58. Nelson, S.M., Biomarkers of ovarian response: current and future applications. Fertil Steril, 2013. 99(4): p. 963-9.

59. Dolleman, M., et al., Reproductive and lifestyle determinants of anti-Mullerian hormone in a large population-based study. J Clin Endocrinol Metab, 2013. 98(5): p. 2106-15.

60. Bhide, P., et al., Serum anti-Mullerian hormone levels across different ethnic groups: a cross-sectional study. BJOG, 2015. 122(12): p. 1625-9.

61. Bleil, M.E., et al., Racelethnic disparities in reproductive age: an examination of ovarian reserve estimates across four racelethnic groups of healthy, regularly cycling women. Fertil Steril, 2014. 101(1): p. 199-207. 
62. Bragg, J.M., et al., Age at menarche and parity are independently associated with Anti-Mullerian hormone, a marker of ovarian reserve, in Filipino young adult women. Am J Hum Biol, 2012. 24(6): p. 739-45.

63. Pigny, P., et al., Elevated serum level of anti-mullerian hormone in patients with polycystic ovary syndrome: relationship to the ovarian follicle excess and to the follicular arrest. J Clin Endocrinol Metab, 2003. 88(12): p. 5957-62.

64. Tal, R., et al., Characterization of women with elevated antimullerian hormone levels (AMH): correlation of AMH with polycystic ovarian syndrome phenotypes and assisted reproductive technology outcomes. Am J Obstet Gynecol, 2014. 211(1): p. 59.e1-8.

65. Chang, H.J., et al., Impact of laparoscopic cystectomy on ovarian reserve: serial changes of serum anti-M\&\#xfc;llerian hormone levels. Fertility and Sterility. 94(1): p. 343-349.

66. Iwase, A., et al., Serum anti-M\&\#xfc;llerian hormone level is a useful marker for evaluating the impact of laparoscopic cystectomy on ovarian reserve. Fertility and Sterility. 94(7): p. 2846-2849.

67. La Marca, A., et al., Anti-Mullerian hormone in premenopausal women and after spontaneous or surgically induced menopause. J Soc Gynecol Investig, 2005. 12(7): p. 545-8.

68. Freeman, E.W., et al., Association of anti-mullerian hormone levels with obesity in late reproductive-age women. Fertility and Sterility. 87(1): p. 101-106.

69. Steiner, A.Z., et al., Antimullerian hormone and obesity: insights in oral contraceptive users. Contraception, 2010. 81(3): p. 245-8. 
70. Buyuk, E., et al., Elevated body mass index is associated with lower serum antimullerian hormone levels in infertile women with diminished ovarian reserve but not with normal ovarian reserve. Fertil Steril, 2011. 95(7): p. 2364-8.

71. Shaw, C.M., et al., Serum antim\&\#xfc;llerian hormone in healthy premenopausal women. Fertility and Sterility. 95(8): p. 2718-2721.

72. Iliodromiti, S., R.A. Anderson, and S.M. Nelson, Technical and performance characteristics of anti-Mullerian hormone and antral follicle count as biomarkers of ovarian response. Hum Reprod Update, 2015. 21(6): p. 698-710.

73. Sahmay, S., et al., Is there any correlation between amh and obesity in premenopausal women? Arch Gynecol Obstet, 2012. 286(3): p. 661-5.

74. Centers for Disease, C., et al., Publications and Reports of the Surgeon General, in How Tobacco Smoke Causes Disease: The Biology and Behavioral Basis for Smoking-Attributable Disease: A Report of the Surgeon General. 2010, Centers for Disease Control and Prevention (US): Atlanta (GA).

75. Dechanet, C., et al., Effects of cigarette smoking on reproduction. Hum Reprod Update, 2011. 17(1): p. 76-95.

76. Sadeu, J.C. and W.G. Foster, Cigarette smoke condensate exposure delays follicular development and function in a stage-dependent manner. Fertil Steril, 2011. 95(7): p. 2410-7.

77. Sadeu, J.C. and W.G. Foster, The cigarette smoke constituent benzo[a]pyrene disrupts metabolic enzyme, and apoptosis pathway member gene expression in ovarian follicles. Reprod Toxicol, 2013. 40: p. 52-9. 
78. Sadeu, J.C. and W.G. Foster, Effect of in vitro exposure to benzo[a]pyrene, a component of cigarette smoke, on folliculogenesis, steroidogenesis and oocyte nuclear maturation. Reprod Toxicol, 2011. 31(4): p. 402-8.

79. Vrsanska, S., et al., Components of cigarette smoke inhibit expansion of oocytecumulus complexes from porcine follicles. Physiol Res, 2003. 52(3): p. 383-7.

80. Massanyi, P., et al., Female reproductive toxicology of cadmium. Acta Biol Hung, 2007. 58(3): p. 287-99.

81. Henson, M.C. and P.J. Chedrese, Endocrine disruption by cadmium, a common environmental toxicant with paradoxical effects on reproduction. Exp Biol Med (Maywood), 2004. 229(5): p. 383-92.

82. Nampoothiri, L.P., A. Agarwal, and S. Gupta, Effect of co-exposure to lead and cadmium on antioxidant status in rat ovarian granulose cells. Arch Toxicol, 2007. 81(3): p. 145-50.

83. Motejlek, K., et al., Smoking impairs angiogenesis during maturation of human oocytes. Fertil Steril, 2006. 86(1): p. 186-91.

84. Jamal, A., et al., Current Cigarette Smoking Among Adults - United States, 20052015. MMWR Morb Mortal Wkly Rep, 2016. 65(44): p. 1205-1211.

85. Centers for Disease Control and Prevention and B.R.F.S. System. Behavioral Risk Factor Surveillance System Prevalence and Trends Data. 2016 [cited 2018 March 11]; Available from: https://www.cdc.gov/brfss/brfssprevalence/index.html.

86. Benowitz, N.L., Biomarkers of environmental tobacco smoke exposure. Environ Health Perspect, 1999. 107 Suppl 2: p. 349-55. 
87. Avila-Tang, E., et al., Assessing secondhand smoke using biological markers. Tob Control, 2013. 22(3): p. 164-71.

88. Benowitz, N.L., et al., Prevalence of smoking assessed biochemically in an urban public hospital: a rationale for routine cotinine screening. Am J Epidemiol, 2009. 170(7): p. 885-91.

89. Balhara, Y.P. and R. Jain, A receiver operated curve-based evaluation of change in sensitivity and specificity of cotinine urinalysis for detecting active tobacco use. J Cancer Res Ther, 2013. 9(1): p. 84-9.

90. Benowitz, N.L., et al., Urine nicotine metabolite concentrations in relation to plasma cotinine during low-level nicotine exposure. Nicotine Tob Res, 2009. 11(8): p. 954-60.

91. Homa, D.M., et al., Vital signs: disparities in nonsmokers' exposure to secondhand smoke--United States, 1999-2012. MMWR Morb Mortal Wkly Rep, 2015. 64(4): p. 103-8.

92. Benowitz, N.L., et al., Optimal serum cotinine levels for distinguishing cigarette smokers and nonsmokers within different racial/ethnic groups in the United States between 1999 and 2004. Am J Epidemiol, 2009. 169(2): p. 236-48.

93. Fuentes, A., et al., [Effects of smoking on plasma antimullerian hormone concentrations among infertile women]. Rev Med Chil, 2013. 141(1): p. 23-7.

94. Plante, B.J., et al., The impact of smoking on antimullerian hormone levels in women aged 38 to 50 years. Menopause, 2010. 17(3): p. 571-6.

95. White, A.J., et al., Antimullerian hormone in relation to tobacco and marijuana use and sources of indoor heating/cooking. Fertil Steril, 2016. 106(3): p. 723-30. 
96. Waylen, A.L., G.L. Jones, and W.L. Ledger, Effect of cigarette smoking upon reproductive hormones in women of reproductive age: a retrospective analysis. Reprod Biomed Online, 2010. 20(6): p. 861-5.

97. Hawkins Bressler, L., et al., Alcohol, cigarette smoking, and ovarian reserve in reproductive-age African-American women. Am J Obstet Gynecol, 2016. 215(6): p. 758 e1-758 e9.

98. Kline, J., A. Tang, and B. Levin, Smoking, alcohol and caffeine in relation to two hormonal indicators of ovarian age during the reproductive years. Maturitas, 2016. 92: p. 115-122.

99. Hein, D.W., Acetylator genotype and arylamine-induced carcinogenesis. Biochim Biophys Acta, 1988. 948(1): p. 37-66.

100. Hein, D.W., et al., Molecular genetics and epidemiology of the NAT1 and NAT2 acetylation polymorphisms. Cancer Epidemiol Biomarkers Prev, 2000. 9(1): p. 29-42.

101. Sim, E., et al., Arylamine N-acetyltransferases: structural and functional implications of polymorphisms. Toxicology, 2008. 254(3): p. 170-83.

102. Hein, D.W., Molecular genetics and function of NAT1 and NAT2: role in aromatic amine metabolism and carcinogenesis. Mutat Res, 2002. 506-507: p. 6577.

103. Hein, D.W., et al., Acetyltransferases and susceptibility to chemicals. Toxicol Lett, 1992. 64-65 Spec No: p. 123-30. 
104. Flamini, G., et al., 4-Aminobiphenyl-DNA adducts in laryngeal tissue and smoking habits: an immunohistochemical study. Carcinogenesis, 1998. 19(2): p. $353-7$.

105. Wang, L.Y., et al., 4-Aminobiphenyl DNA damage in liver tissue of hepatocellular carcinoma patients and controls. Am J Epidemiol, 1998. 147(3): p. 315-23.

106. Curigliano, G., et al., Immunohistochemical quantitation of 4-aminobiphenylDNA adducts and p53 nuclear overexpression in 11 bladder cancer of smokers and nonsmokers. Carcinogenesis, 1996. 17(5): p. 911-6.

107. Airoldi, L., et al., Determinants of 4-aminobiphenyl-DNA adducts in bladder cancer biopsies. Carcinogenesis, 2002. 23(5): p. 861-6.

108. Zhu, J., et al., Detection of 2-amino-1-methyl-6-phenylimidazo[4,5-b]-pyridineDNA adducts in normal breast tissues and risk of breast cancer. Cancer Epidemiol Biomarkers Prev, 2003. 12(9): p. 830-7.

109. Gorlewska-Roberts, K., et al., Carcinogen-DNA adducts in human breast epithelial cells. Environ Mol Mutagen, 2002. 39(2-3): p. 184-92.

110. Faraglia, B., et al., Evaluation of 4-aminobiphenyl-DNA adducts in human breast cancer: the influence of tobacco smoke. Carcinogenesis, 2003. 24(4): p. 719-25.

111. Ambrosone, C.B., et al., Hair dye use, meat intake, and tobacco exposure and presence of carcinogen-DNA adducts in exfoliated breast ductal epithelial cells. Arch Biochem Biophys, 2007. 464(2): p. 169-75.

112. Besaratinia, A., et al., Immunoperoxidase detection of 4-aminobiphenyl-and polycyclic aromatic hydrocarbons-DNA adducts in induced sputum of smokers and non-smokers. Mutat Res, 2000. 468(2): p. 125-35. 
113. Bendaly, J., et al., Differences between human slow N-acetyltransferase 2 alleles in levels of 4-aminobiphenyl-induced DNA adducts and mutations. Mutat Res, 2009. 671(1-2): p. 13-9.

114. Walraven, J.M., et al., Structure/function evaluations of single nucleotide polymorphisms in human N-acetyltransferase 2. Curr Drug Metab, 2008. 9(6): p. 471-86.

115. Vatsis, K.P., et al., Nomenclature for N-acetyltransferases. Pharmacogenetics, 1995. 5(1): p. 1-17.

116. Supplementary Table 2. Distribution and annotation of NAT genes in Humans. [cited 2017 October 19]; Available from: http://nat.mbg.duth.gr/Human\%20NAT2\%20alleles_2013.htm\#_Footnotes.

117. Hein, D.W., N-acetyltransferase SNPs: emerging concepts serve as a paradigm for understanding complexities of personalized medicine. Expert Opin Drug Metab Toxicol, 2009. 5(4): p. 353-66.

118. Lin, H.J., et al., Slow acetylator mutations in the human polymorphic $N$ acetyltransferase gene in 786 Asians, blacks, Hispanics, and whites: application to metabolic epidemiology. Am J Hum Genet, 1993. 52(4): p. 827-34.

119. Garcia-Martin, E., Interethnic and intraethnic variability of NAT2 single nucleotide polymorphisms. Curr Drug Metab, 2008. 9(6): p. 487-97.

120. Gross, M., et al., Distribution and concordance of $N$-acetyltransferase genotype and phenotype in an American population. Cancer Epidemiol Biomarkers Prev, 1999. 8(8): p. 683-92. 
121. Garte, S., et al., Metabolic gene polymorphism frequencies in control populations. Cancer Epidemiol Biomarkers Prev, 2001. 10(12): p. 1239-48.

122. Zang, Y., et al., Functional characterization of single-nucleotide polymorphisms and haplotypes of human N-acetyltransferase 2. Carcinogenesis, 2007. 28(8): p. $1665-71$.

123. Marcus, P.M., et al., Cigarette smoking, N-acetyltransferase 2 acetylation status, and bladder cancer risk: a case-series meta-analysis of a gene-environment interaction. Cancer Epidemiol Biomarkers Prev, 2000. 9(5): p. 461-7.

124. Garcia-Closas, M., et al., NAT2 slow acetylation, GSTM1 null genotype, and risk of bladder cancer: results from the Spanish Bladder Cancer Study and metaanalyses. Lancet, 2005. 366(9486): p. 649-59.

125. Risch, A., et al., Slow $N$-acetylation genotype is a susceptibility factor in occupational and smoking related bladder cancer. Hum Mol Genet, 1995. 4(2): p. 231-6.

126. Moslehi, R., et al., Cigarette smoking, $N$-acetyltransferase genes and the risk of advanced colorectal adenoma. Pharmacogenomics, 2006. 7(6): p. 819-29.

127. Wikman, H., et al., Relevance of $N$-acetyltransferase 1 and 2 (NAT1, NAT2) genetic polymorphisms in non-small cell lung cancer susceptibility. Pharmacogenetics, 2001. 11(2): p. 157-68.

128. Ambrosone, C.B., et al., Cigarette smoking, $\mathrm{N}$-acetyltransferase 2 genetic polymorphisms, and breast cancer risk. Jama, 1996. 276(18): p. 1494-501.

129. Hunter, D.J., et al., A prospective study of NAT2 acetylation genotype, cigarette smoking, and risk of breast cancer. Carcinogenesis, 1997. 18(11): p. 2127-32. 
130. Goode, E.L., et al., Xenobiotic-Metabolizing gene polymorphisms and ovarian cancer risk. Mol Carcinog, 2011. 50(5): p. 397-402.

131. Mendola, P., et al., Risk of recurrent spontaneous abortion, cigarette smoking, and genetic polymorphisms in NAT2 and GSTM1. Epidemiology, 1998. 9(6): p. $666-8$.

132. Baranova, H., et al., Possible involvement of arylamine $\mathrm{N}$-acetyltransferase 2, glutathione S-transferases M1 and T1 genes in the development of endometriosis. Mol Hum Reprod, 1999. 5(7): p. 636-41.

133. Centers for Disease Control and Prevention, et al., Assisted Reproductive Technology Fertility Clinic Success Rates Report Atlanta (GA): US Dept of Health and Human Services; 2015. 2015: p. 236.

134. Kara, M., et al., Does dehydroepiandrosterone supplementation really affect IVFICSI outcome in women with poor ovarian reserve? Eur J Obstet Gynecol Reprod Biol, 2014. 173: p. 63-5.

135. World Health Organization. Global Database on Body Mass Index; BMI classification. . 05/02/2018 [cited 2018 February 5]; Available from: http://apps.who.int/bmi/index.jsp?introPage=intro_3.html.

136. Centers for Disease Control and Prevention. National Center for Health Statistics: NHANES. Questionnaires, Datasets, and Related Documentation. October 30, 2015 [cited 2018 February 8]; Available from:

https://www.cdc.gov/nchs/nhanes/nhanes_questionnaires.htm.

137. Centers for Disease Control and Prevention. National Center for Health Statistics: National Health Interview Survey. Questionnaires, Datasets, and Related 
Documentation. June 26, 2017 [cited 2018 February 8]; Available from:

https://www.cdc.gov/nchs/nhis/nhis_questionnaires.htm.

138. Hein, D.W. and M.A. Doll, Accuracy of various human NAT2 SNP genotyping panels to infer rapid, intermediate and slow acetylator phenotypes.

Pharmacogenomics, 2012. 13(1): p. 31-41.

139. Doll, M.A. and D.W. Hein, Comprehensive human NAT2 genotype method using single nucleotide polymorphism-specific polymerase chain reaction primers and fluorogenic probes. Anal Biochem, 2001. 288(1): p. 106-8.

140. Hein, D.W. and M.A. Doll, TaqMan real time-polymerase chain reaction methods for determination of nucleotide polymorphisms in human $\mathrm{N}$-acetyltransferase- 1 (NAT1) and -2 (NAT2). Curr Protoc Toxicol, 2005. Chapter 4: p. Unit4.15.

141. Pallam, H., et al., Louisville Metro Behavioral Risk Factor Surveillance Survey Results, L.D.o.P.H.a. Wellness, Editor. 2016.

142. Oboni, J.B., et al., Impact of smoking on fertility and age of menopause: a population-based assessment. BMJ Open, 2016. 6(11): p. e012015.

143. Schoenaker, D.A., et al., Socioeconomic position, lifestyle factors and age at natural menopause: a systematic review and meta-analyses of studies across six continents. Int J Epidemiol, 2014. 43(5): p. 1542-62.

144. Sun, L., et al., Meta-analysis suggests that smoking is associated with an increased risk of early natural menopause. Menopause, 2012. 19(2): p. 126-32.

145. Parente, R.C., et al., The relationship between smoking and age at the menopause: A systematic review. Maturitas, 2008. 61(4): p. 287-98. 
146. Midgette, A.S. and J.A. Baron, Cigarette smoking and the risk of natural menopause. Epidemiology, 1990. 1(6): p. 474-80.

147. Wang, S., et al., Influence of arylamine $\mathrm{N}$-acetyltransferase, sex, and age on 4aminobiphenyl-induced in vivo mutant frequencies and spectra in mouse liver. Environ Mol Mutagen, 2012. 53(5): p. 350-7.

148. Welsh, P., K. Smith, and S.M. Nelson, A single-centre evaluation of two new antiMullerian hormone assays and comparison with the current clinical standard assay. Hum Reprod, 2014. 29(5): p. 1035-41.

149. Burks, H.R., et al., Can highly sensitive antimullerian hormone testing predict failed response to ovarian stimulation? Fertil Steril, 2015. 104(3): p. 643-8. 


\section{Appendix 1}

\section{Supplemental Smoking and Hypertension Questionnaire}

Sample of questionnaire designed to assess smoking history and Secondhand smoke exposure 


\section{LOUSSI SUPPLEMENTAL SMOKING QUESTIONNAIRE \\ LIFETIME EXPOSURE TO TOBACCO SMOKE \\ INSTRUCTIONS}

\section{Dear Participant:}

Thank you for taking the time to answer this questionnaire. This form should take 5 to 10 minutes to complete. Your answers will be used for research purposes only. Your doctor will not know the answers you have marked. Information gained from this study will help scientists and doctors understand the effect of smoking and secondhand smoke on fertility.

This packet contains three questionnaires.

Please choose $\mathrm{ONE}$ questionnaire to complete, depending on whether you are a current smoker, former smoker, or nonsmoker. Leave the other two questionnaires blank.

CURRENT SMOKERS: Please answer Questionnaire 1. You are considered a current smoker if you currently smoke at least 1 cigarette/week, or if you recently quit (less than 1 month ago)

FORMER SMOKERS: Please answer Questionnaire 2. You are considered a former smoker if you quit more than 1 month ago and used to smoke at least 1 cigarette/week.

NONSMOKERS: Please answer Questionnaire 3. You are considered a nonsmoker if you have never smoked more than 1 cigarette/week.

Please place questionnaire in the envelope provided and return it to the study personnel or place it inside the designated LOUSSI Study dropbox when you are finished.

Thank you very much for your time!

QUESTIONNAIRE 1 (FOR CURRENT SMOKERS)

Answer this questionnaire if you currently smoke at least 1 cigarette/week, 


\section{or if you quit smoking less than 1 month ago.}

\section{Smoking History}

1. How many years have you been smoking (at least 1 cigarette/week)? years

(If you have smoked on and off, please add up the total number of years you smoked.)

2. On the average, how many cigarettes (or packs) do you now smoke per day?

cigarettes/day or packs/day

(If you quit less than 1 month ago, please indicate how much you used to smoke before you quit.)

\section{Secondhand Smoke}

3. Think about your home when you were growing up (less than $\mathbf{1 8}$ years old). On average, how often were you exposed to secondhand smoke inside your home? (For example, how often did your parents, guardians, or siblings smoke inside the home?)

Never Rarely Often Every day

(less than once/week) (1-6 days/week)

4. Think about your home in the past year. How often have you been exposed to secondhand smoke inside your home?
Never
Rarely
Often
Every day

(less than once/week) (1-6 days/week)

5. Think about other places you go on a regular basis. (For example, a friend or relative's house, your workplace, bars or restaurants, in your car or someone else's car, etc.) In the past year, how often have you been exposed to secondhand smoke inside other places besides your home?

Never Rarely Often Every day

(less than once/week) (1-6 days/week) 
back $\rightarrow$

6. Does anyone else living in your home smoke cigarettes, cigars or a pipe?

$\square$ Yes

$\square$ No

If you answered "Yes", how many other people living in your home smoke these tobacco products?

Enter number

What is your relationship with the smoker(s)? (Check all that apply)

Spouse or partner

Other

\section{Other sources of nicotine}

7. Please check whether you have used any of the following in the past week:

Nicotine patches

Nicorette gum or similar

E-cigarettes

Smokeless tobacco or chewing tobacco

Other source of nicotine: 
QUESTIONNAIRE 2 (FOR FORMER SMOKERS)

\section{Answer this questionnaire if you used to smoke at least 1 cigarette/week and quit} at least 1 month ago.

\section{Past Smoking}

1. When is the last time you smoked a cigarette? (Month, Year)

2. How many years did you smoke at least 1 cigarette/week? years

(If you smoked on and off, please add up the total number of years you smoked.)

day?

3. On the average, how many cigarettes (or packs) did you used to smoke per

cigarettes/day or packs/day

\section{Secondhand Smoke}

4. Think about your home when you were growing up (less than 18 years old). On average, how often were you exposed to secondhand smoke inside your home? (For example, how often did your parents, guardians, or siblings smoke inside the home?)
Never
Rarely
Often
Every day
(less than once/week) (1-6 days/week)

5. Think about your home in the past year. How often have you been exposed to secondhand smoke inside your home?
Never
Rarely
Often
Every day
(less than once/week) (1-6 days/week)

6. Think about other places you go on a regular basis. (For example, a friend or relative's house, your workplace, bars or restaurants, in your car or someone else's car, etc.) In the past year, how often have you been exposed to secondhand smoke inside other places besides your home?
Never
Rarely
Often
Every day
(less than once/week) (1-6 days/week) 
back $\rightarrow$

7. Does anyone living in your home smoke cigarettes, cigars or a pipe?

$\square$ Yes

$\square$ No

If you answered "Yes", how many people living in your home smoke these tobacco products?

Enter number

What is your relationship with the smoker(s)? (check all that apply)

Spouse or partner

Other

\section{Other sources of nicotine}

8. Please check whether you have used any of the following in the past week:

Nicotine patches

Nicorette gum or similar

E-cigarettes

Smokeless tobacco or chewing tobacco

Other source of nicotine: 


\section{QUESTIONNAIRE 3 (FOR NONSMOKERS)}

\section{Answer this if you have never smoked more than 1 cigarette/week}

The goal of this questionnaire is to determine how much exposure to secondhand smoke you have had in your lifetime.

\section{Secondhand Smoke}

1. Think about your home when you were growing up (less than $\mathbf{1 8}$ years old). On average, how often were you exposed to secondhand smoke inside your home? (For example, how often did your parents, guardians, or siblings smoke inside your home?)

Never Rarely Often Every day

(less than once/week) (1-6 days/week)

2. Think about your home in the past year. How often have you been exposed to secondhand smoke inside your home?

Never

Rarely

Often

Every day

(less than once/week) (1-6 days/week)

3. Think about other places you go on a regular basis. (For example, a friend or relative's house, your workplace, bars or restaurants, in your car or someone else's car, etc.) In the past year, how often have you been exposed to secondhand smoke inside other places besides your home?
Never
Rarely
Often
Every day
(less than once/week) (1-6 days/week)

4. Does anyone living in your home smoke cigarettes, cigars or a pipe?
Yes
$\square$ No 
If you answered "Yes", how many people living in your home smoke these tobacco products?

\section{Enter number}

What is your relationship with the smoker(s)? (check all that apply)

\section{Spouse or partner}

back $\rightarrow$ Other

Please see

\section{Other sources of nicotine}

5. Please check whether you have used any of the following in the past week:

Nicotine patches

Nicorette gum or similar

E-cigarettes

Smokeless tobacco or chewing tobacco Other source of nicotine: 
Appendix 2

Data Collection Forms

Sample of data entry form for past medical and social history; anthropometric measures;

blood pressure and ovarian reserve measures 
1. Self-reported data From most recent Patient History Form (New patient info) Current tobacco use (yes/no):

Date of first visit (MM/YY):

Date of study enrollment (MM/YY):

Age (years) at enrollment:

Height: (feet, inches): feet inches

Weight: pounds or $\mathrm{kg}$

Body mass index: $\mathrm{kg} / \mathrm{m}^{2}$

Total number of previous pregnancies:

Regular periods (yes/no):

Average menstrual cycle length: days

Age at first period: years

Sexually transmitted infections: (yes/no)

If yes, list:

"Social History" Section:

Occupation:

Married/single:

Partner/no partner?

Length of time with current partner:

Routine exercise (yes/no):

Routine exposure to chemicals(Yes/no):

If yes, list here:

Diet restrictions:

Meals/day:
Packs/day

for how many years?

Ever smoked 100 cigarettes?

Alcohol (yes/no)

Alcoholic drinks/week

Caffeine (yes/no)

Caffeinated drinks/day

Drugs (e.g. marijuana) (yes/no)

Ancestry or Race

Consistent ovulation (yes/no) 
Appendix 3

Patient History Forms

Sample of survey filled by patients during first clinic visit 


\section{UL Physicians}

PATIENT INFORMATION FORU

Patient Information Name

Aso Known As

SSN

Date of Bith

Sex $\square$ Male $\square$ Fende

Marital Stabus $\square$ single $\square$ Uamied $\square$ Divorced $\square$ Widowed $\square$ Seperabed Preferred Language

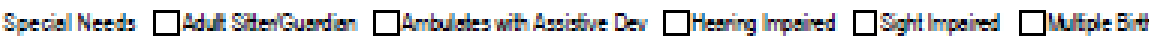

$\square$ Speech Impoived $\square$ Wheelchair $\square$ inteprebe $\quad \square$ Trensportation Needs

Pabent Race: Roce- a humen population considered distind besed on physical charocteristics.

$\square$ American Indian $\square$ Alaska Nabive

$\square$ Asion $\square$ Black or African Americs-

White $\square$ Nafive Hawsian or Other Pacific blander

$\square$ Other $\quad \square$ Declined

Ethnicily: Ehricity a tem which represerts social groups with a shaved history, serse of iderttly, geogrephy and alhural roots which moy occur despite recial dfference.

$\square$ Hispanic or Labino $\square$ Not Hispenic or Latino

Religion

Home Addres:

Cty, St Courty Zip Code

Home Phone Cel Phone Work Other Phone

Emai

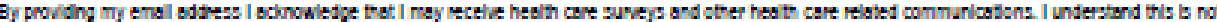
to be used for povider commuricason and thet errali le not seaus and can be intercepled and used ty ursuthorzed pergons.

Employment Statu: Employer Name

Employer Phone Employer Address

Employer City, St Zp Code

Pimsry Physician Primary Physicisn Phone

Referring Physician Referring Physician Phone

Preferred Phomacy Phamacy Phone

Phamscy Address, City, St, Zp

Parent/Guardian(a) or Spouse Information Name

Relationship to Patent SSN Date of Bith

Address (If Diferent] Zip Code

Employer Employer Phone

Employer Address $\mathrm{Zp} \cos e$

Home Phone Emsil

nreos

Thined Pabruavy 10, 2006 


\title{
UEL Physicians \\ OB/GYN \& WOMEN'S HEALTH
}

\author{
401 E. Chesinut Street, Sultes 400,410 \& 470 \\ Louisvile, KY 40202
}

$\{502,598-4400$

Parient Labe!

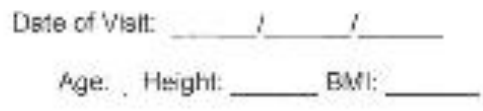

\section{New Patient History}

Please note: All information is confldential and will only be used for the purpose of ensuring you the best treatment possible. Pleasc answer all arcas:

\begin{tabular}{l} 
Why have you come to the office tarlay? \\
\hline \\
\hline Are you hann? any problems? Where? How bng have you had the problemis)? \\
\hline \\
Who referred you to our practice? a Selt a Friend c Physician (Print Narre) \\
\hline
\end{tabular}

Who is your usual OblGyn?

Location:

Past Medlcal Histon (wi if you have or have ever had)

\begin{tabular}{|c|c|c|c|c|c|}
\hline & $\because$ & & $j$ & & 13 \\
\hline Anemiablood disease & & Gal bladder disease & & Price biad elots (CVTs) & \\
\hline Arthits & & Headachesirnigraine & & Refluxi'Hiatal Hornia & \\
\hline Astheria: & & Heart disearenivalve probems & & Skin disease & \\
\hline $\begin{array}{l}\text { Blaudiei } \\
\text { problemónfections }\end{array}$ & & High bland presssure & & Stomech woor & \\
\hline Elcod translusion & & Kidhey disease & & Thyroid diseaso & \\
\hline Econel disordier & & Leukerria & & Tukercubas & \\
\hline Cianoer & & I wer cliseascinepabtis & & Veriocse vensighlevibs & \\
\hline Dianates & & Lung disesse & & Weight lossigsin $\geq 10 \mathrm{lbs}$ & \\
\hline Endometriosis & & $\begin{array}{l}\text { PCOS iPolycysto Ovarian } \\
\text { Syndromel }\end{array}$ & & & \\
\hline $\begin{array}{l}\text { Epiepsyineurolegic } \\
\text { desense }\end{array}$ & & Prior Pulmonary Embslus (PE) & & & \\
\hline & & & & & \\
\hline \multicolumn{6}{|c|}{ Other modical problems (list): } \\
\hline
\end{tabular}

Page 1 of ? 
SurgerlesiOperations (Any procedure, including D\&C's)

\begin{tabular}{|l|l|l|l|}
\hline TypeiReason & Date & \multicolumn{1}{|c|}{ Location Physician } \\
\hline & & & \\
\hline & & & \\
\hline & & & \\
\hline & & & \\
\hline
\end{tabular}

Other IllnessesiHospitalizations

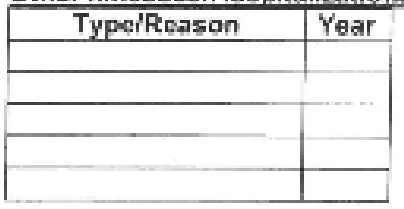

Current Medications

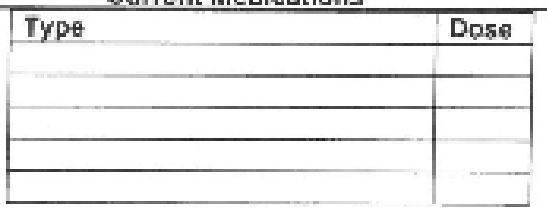

Food or drugg allergles

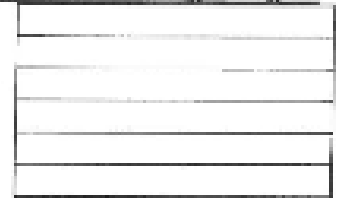

Hlstory

\begin{tabular}{|c|c|}
\hline First dey of last menstual period: & \multirow{2}{*}{$\begin{array}{l}\text { Hawe you ever had an sbrormal Pap test? } \\
\text { Yes } \square \mathrm{No}\end{array}$} \\
\hline $\begin{array}{l}\text { Do wou have regular monthly perisds? } \\
\text { Yes } 5 \text { No o }\end{array}$ & \\
\hline $\begin{array}{l}\text { Usubi number of days from start of one period ta } \\
\text { start of the next }\end{array}$ & $\begin{array}{l}\text { Have you suer had a procedure on your cervix due to } \\
\text { an abnomal pap test? (LEEP. Cryoicone) } \\
\text { Yes a No } \mathrm{Y} \text { Year: }\end{array}$ \\
\hline $\begin{array}{l}\text { Any recent dianges? } \\
\text { Yes a No } a \\
\text { Describe: }\end{array}$ & $\begin{array}{l}\text { When was your last Pap test? } \\
\text { Whet was the Pap result? } \\
\text { ONormal J Abnonnal }\end{array}$ \\
\hline Number of days of bleeding. & \multirow{2}{*}{$\begin{array}{l}\text { Have you ever had a mammogram? } \\
\text { Yes a Na J } \\
\text { Year of last marnogram:- } \\
\text { L Nomal } \quad \text { Abnomal } \\
\end{array}$} \\
\hline Ago periads began: & \\
\hline $\begin{array}{l}\text { Do you have prablems with pelvis pein? } \\
\text { Yes u No } u \\
\text { When? }\end{array}$ & \multirow{4}{*}{ 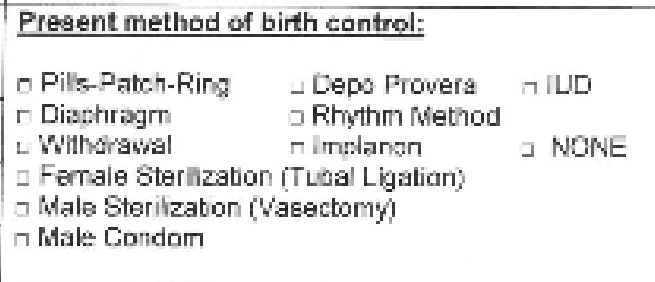 } \\
\hline $\begin{array}{l}\text { Are you sexuglly sctive? } \\
\text { Yes } \square \text { No }\end{array}$ & \\
\hline $\begin{array}{l}\text { Do you have pain with intercourse? } \\
\text { Yes } \square \mathrm{No} \mathrm{a}\end{array}$ & \\
\hline $\begin{array}{l}\text { Sexual partner(s) isiare: } \\
\text { Men a Wamen o Bcth c }\end{array}$ & \\
\hline $\begin{array}{l}\text { Have you ever hed any of the following infections? } \\
\neg \text { Gonarthes } 7 \text { Chlemydia } 5 \text { Herpes } \\
\text { HPV }\end{array}$ & $\begin{array}{l}\text { Weight is age } 20 \\
\text { Current Weight: }\end{array}$ \\
\hline Number of times: _____ Yearisi: ___ & $\begin{array}{l}\text { Have you eve wsed Birth Control Pills? } \\
\text { No } ~ \\
\text { Age whon started birth controt. } \\
\text { Age when last stopped: }\end{array}$ \\
\hline
\end{tabular}

Page 2 af 7 


\section{Hirsutism (excessive hair arowth) \& Acne}

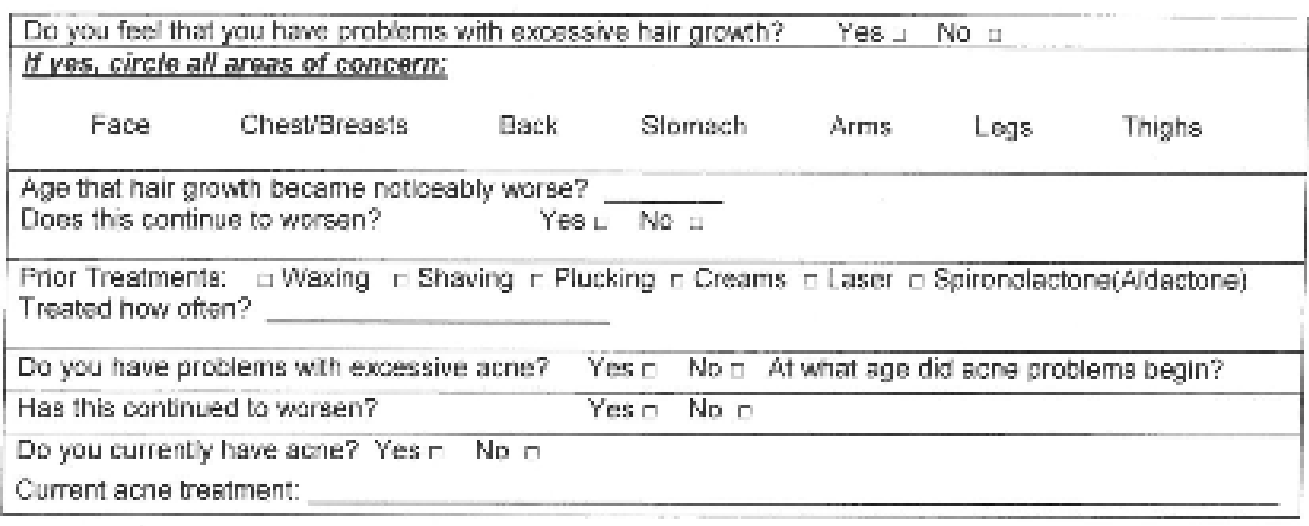

\section{Obstotrical History}

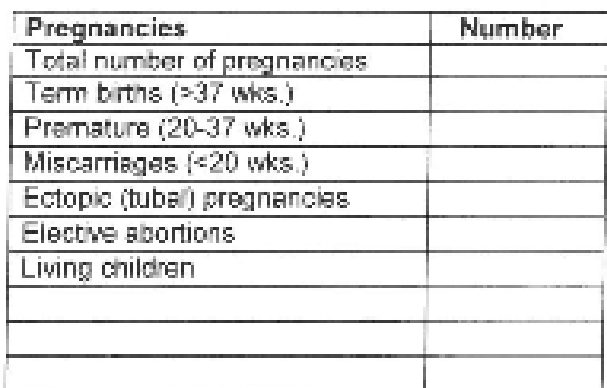

Immunizations

\begin{tabular}{|c|c|}
\hline \multirow{2}{*}{\multicolumn{2}{|c|}{$\begin{array}{l}\text { Type of Immunizatlon } \\
\text { Heve you ever had Chicken Pox? }\end{array}$}} \\
\hline & \\
\hline Chicken pax vaccine & \\
\hline Hepatibs A vaccine & \\
\hline Hepsiltis B veccine & \\
\hline RubellaiMMR vaccine & \\
\hline HPy vaccire (Gardasil) & \\
\hline TB test & \\
\hline Flu veccine & \\
\hline Pneumonis vaccine & \\
\hline Other: & \\
\hline
\end{tabular}

\section{Obstatrical Histary: Please list all pregnancles in order}

\begin{tabular}{|c|c|c|c|c|c|c|c|c|c|c|}
\hline & \multirow[b]{2}{*}{$\begin{array}{l}\text { Morth } \\
\text { Yhar }\end{array}$} & \multicolumn{4}{|c|}{ Oubceme (Yes/No) } & \multirow{2}{*}{$\begin{array}{l}\text { Doliwary } \\
\text { Vaje- } \\
\text { Sation }\end{array}$} & \multirow[b]{2}{*}{ Compricsters } & \multirow{2}{*}{$\begin{array}{l}\text { Lewgrief } \\
\text { Tire to } \\
\text { Cunceines }\end{array}$} & \multirow{2}{*}{$\begin{array}{l}\text { Reg-ared } \\
\text { totil ty } \\
\text { Treatment }\end{array}$} & \multirow{2}{*}{$\begin{array}{c}\text { Cutent } \\
\text { Parner } \\
7\end{array}$} \\
\hline & & $\begin{array}{l}\text { Juc } \\
\text { born }\end{array}$ & $M \operatorname{ses} \approx \mathrm{gc}$ & Abation & Enopia & & & & & \\
\hline \multicolumn{11}{|l|}{$1^{31}$} \\
\hline $2^{75}$ & & & & & & & & & & \\
\hline $3^{d}$ & & & & & & & & & & \\
\hline $4^{\text {th }}$ & & & & & & & & & & \\
\hline $5^{1+}$ & & & & & & & & & & \\
\hline $6^{a^{-2}}$ & & & & & & & & & & \\
\hline 7th & & & & & & & & & & \\
\hline
\end{tabular}




\title{
Soclal Hlstory:
}

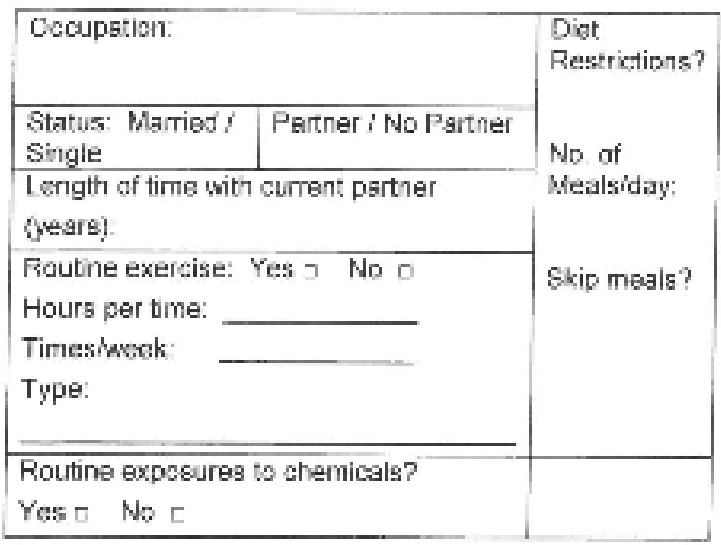

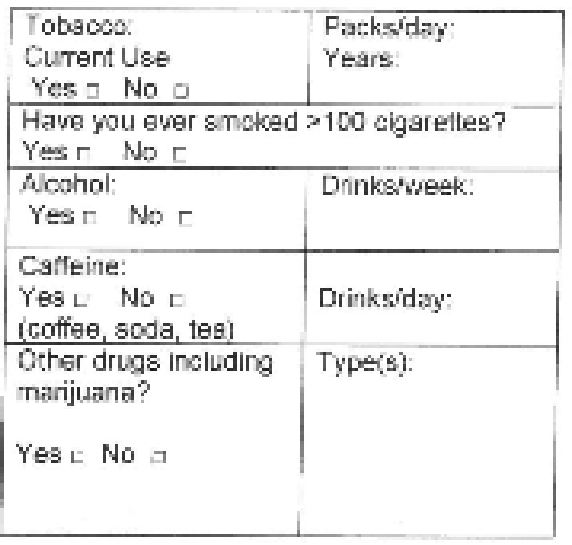

\section{Genetic Diserses:}

\begin{abstract}
Cystic Fibrosls
Oystic Fibrosis (CFI s a hereditary disease that affects mainly the lungs and digeabwe syetem. causing progressive disabilty, recurnent infecbons and usuelly early death. CF does not affect intelligence or apcearance. Averiage life expertancy is around 37 years. Approwmately 1 in 29 Cascasans c6Ty this gene defect, as well as 1 in 46 Hisparics, and 1 in 65 African Amencang. If you are a carrier, you hawe a $50 \%$ chances of your child being a camier, which would not tae affected if your carther is elso $\mathrm{a}$ carner, you have a $25 \%$ chance of having a child wh the disease. The American Colkge of Obsteticians and Gynecolsgists recommends offering all patients testng for CF. Cument testing can determine if you cary the geneis; responsible for this disease. Current testing can detect the genes that are respwrsible far $>90 \%$ of cases of CF. Testing may not be covered by your insuranco. The tes: costs approxmately $\$ 395$ to perform if net cowered $\mathrm{byg}_{\mathrm{g}}$ insurance. If you do not have insurance coverage for the you may be able to cocrdingte a reduced fee with bur laboratory if you arrange ths before having the lest done

Have you becn tosted for Cystlc FIbrosis (CF)? Yes - $\mathrm{Na}-$

Would you like to be tested: Yes n No c
\end{abstract}

Family History (Farents, Grandpsrents, Siblings, Auntsuncles)

\begin{tabular}{|c|c|c|}
\hline IIIness & v & List affected relative(s) and age at onset \\
\hline Aleahed or diug adiction & & \\
\hline Birth defertsoldental retardstion & & \\
\hline Blesding disorders & & \\
\hline Elood dots in lunge or legs & & \\
\hline Breast cancer & & \\
\hline Cancer-Colon & & \\
\hline Cancer-Ovary & & \\
\hline Cancer-Llerus & & \\
\hline Disbefees & & \\
\hline Endomatriosis & & \\
\hline
\end{tabular}

Page 4 af 7 


\begin{tabular}{|c|c|}
\hline \multicolumn{2}{|l|}{ Heart disease } \\
\hline \multicolumn{2}{|l|}{ Hepstitis } \\
\hline \multicolumn{2}{|l|}{ High biood pressure } \\
\hline \multicolumn{2}{|l|}{ High cholesterol } \\
\hline \multicolumn{2}{|l|}{$\mathrm{HIV}$} \\
\hline \multicolumn{2}{|l|}{ Infertifty } \\
\hline \multicolumn{2}{|l|}{ Mental illocssidopression } \\
\hline \multicolumn{2}{|l|}{ Osteoporosis (woak bones) } \\
\hline \multicolumn{2}{|l|}{ Sickle Celi Thalssaemis } \\
\hline \multicolumn{2}{|l|}{ Stroke } \\
\hline \multicolumn{2}{|l|}{ Tubercuiosis } \\
\hline \multicolumn{2}{|l|}{ Any other genetic dsesses } \\
\hline Recurfenl miscarriages? & List miscarriages for both your temly's sice and you partner's \\
\hline $\begin{array}{l}\text { Early menopause }<40 \text { years old } \\
\text { (Premature Ovarian Failure) }\end{array}$ & Age of mother st menopause if known: \\
\hline \multicolumn{2}{|l|}{ Other: } \\
\hline 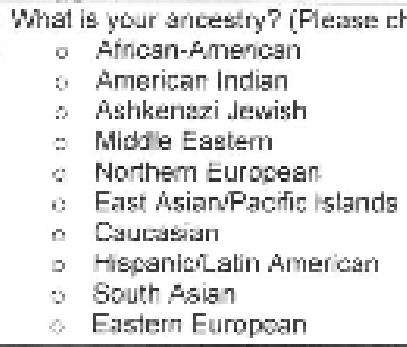 & \\
\hline
\end{tabular}

\section{(You Nay sToP here if not haing seen for fertilly reasons)}

\section{Fertility History}

Note: in poder to help us more efficiently treat you, clease obtain copies of your past fertilty treatrnents. operalive reports, IVF cycke, ultraspund reports, labs and hand sesies. (FIlms or on disk) of any Hyeterosalpingogram (HSG) (X-ray test of your tubes) that you have had done. It is important thet we review the HSG tilms that were previously done. Plesse bring these rocords to vour anoointment wotth vou.

\begin{tabular}{|c|c|}
\hline $\begin{array}{l}\text { How bong have you been actwely trying to conceive? } \\
\text { yrs }\end{array}$ & $\begin{array}{l}\text { Do you use lubricarts? Yos o No } 4 \\
\text { Tyce: }\end{array}$ \\
\hline Number of times of intercourse per visek? & Do you douche? $\quad$ Yeg L No a \\
\hline $\begin{array}{l}\text { How long have you taem eff arny tirth cortrol? } \\
\text { yrs mo. }\end{array}$ & Frequency of intercourse negr avulation. \\
\hline
\end{tabular}

Do you have insurance crworage for fertility? Yes L No L

\section{Iype of insurance benefit:}

[ Testingidiagnosis only

T Testing \& trestment

$\neg$ Artilicial insemination

$\checkmark$ IVF 
Prior Fertility EvaluationiLabs/Treatment

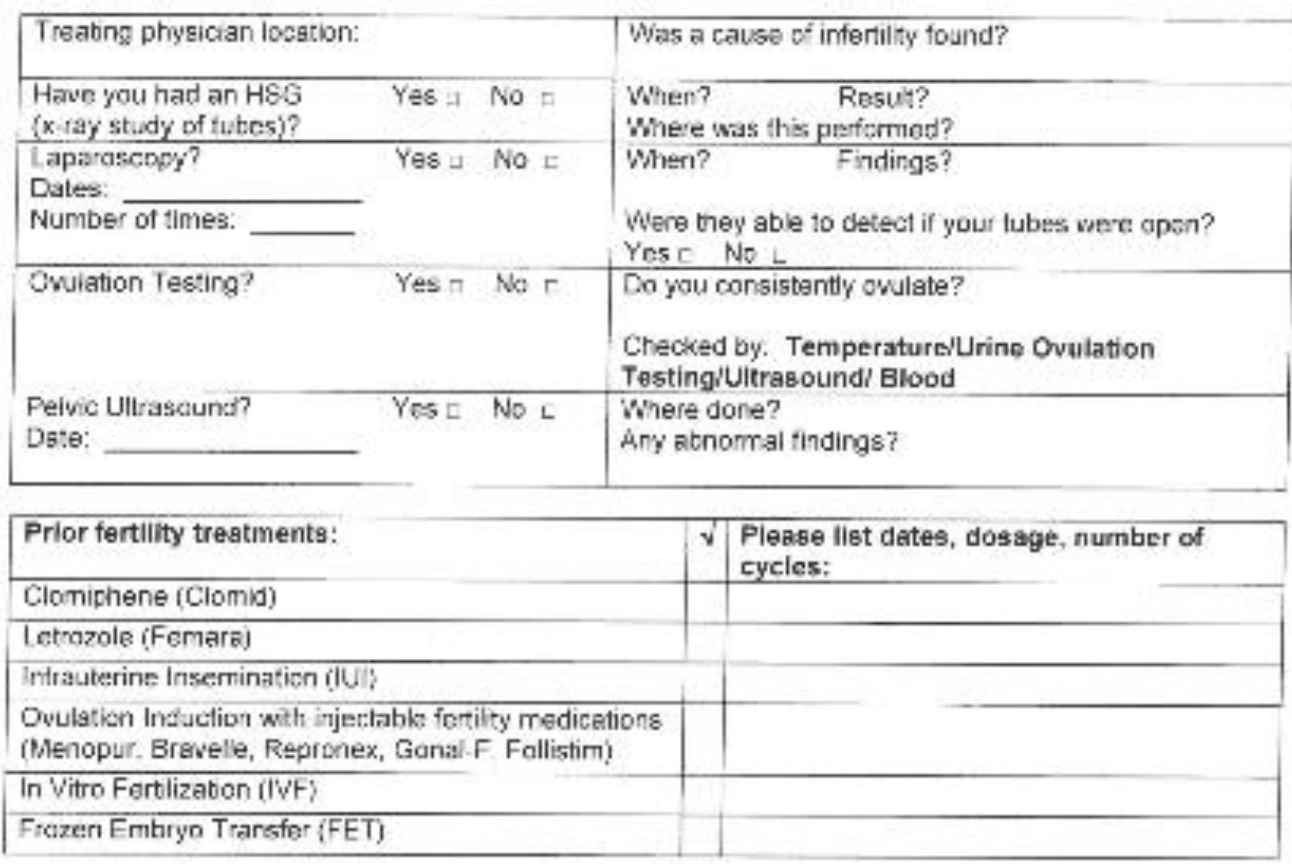

\section{Male Partner History}

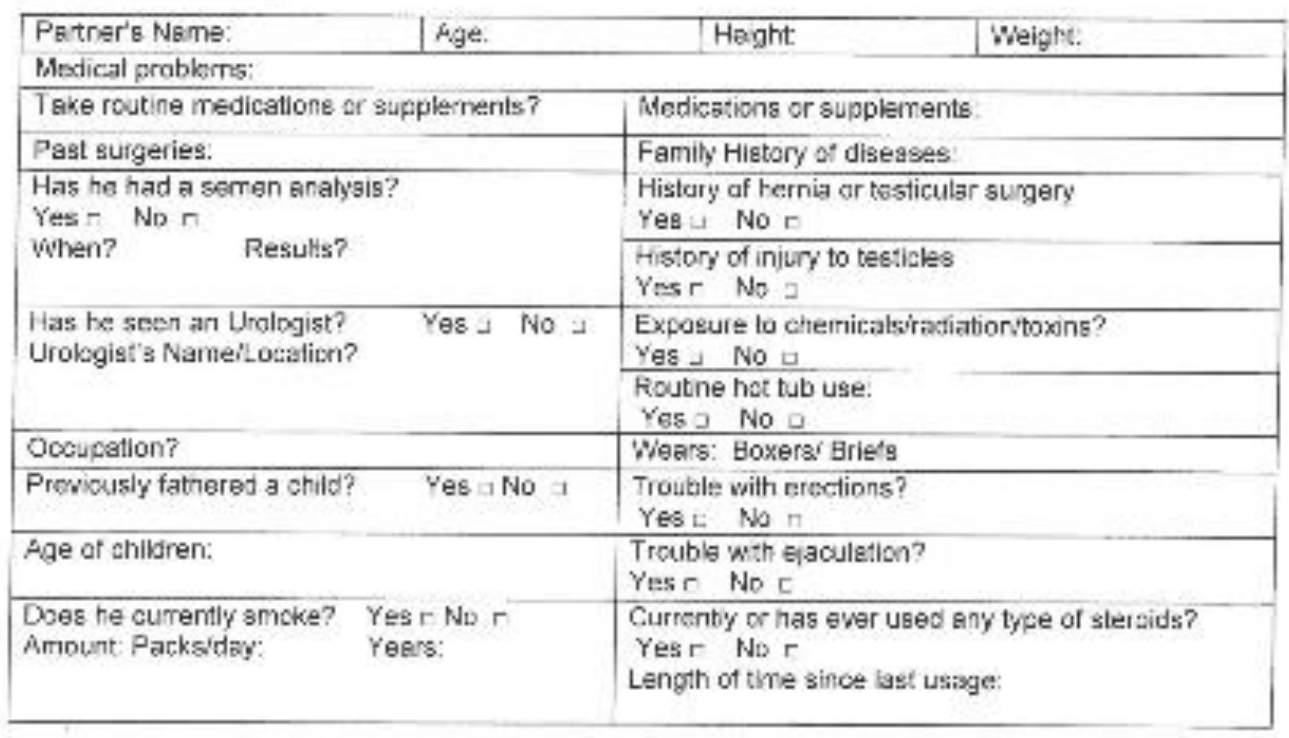




\begin{tabular}{|c|c|}
\hline $\begin{array}{l}\text { Use marijuana or other drugs? Yes } \square \text { No } \square \\
\text { Last use: }\end{array}$ & $\begin{array}{l}\text { Any illnesses/fevers in the past } 3 \text { months? } \\
\text { Yes } \square \text { No } \square\end{array}$ \\
\hline Alcohol use: & $\begin{array}{l}\text { History of sexually transmitted diseases? } \\
\text { Yes } \square \text { No }\end{array}$ \\
\hline \multicolumn{2}{|l|}{ What is your ancestry? (Please check) } \\
\hline - African-American & \\
\hline - American Indian & \\
\hline - Ashkenazi Jewish & \\
\hline - Middle Eastern & \\
\hline - Northern European & \\
\hline - East Asian/Pacific Islands & \\
\hline - Caucasian & \\
\hline - Hispanic/Latin American & \\
\hline South Asian & \\
\hline Castemil curopedan & \\
\hline
\end{tabular}




\section{CURRICULUM VITAE}

\section{CONTACT INFORMATION}

Islamiat A. Oladipupo

Department of Epidemiology and Population Health

University of Louisville, 485 E Gray St

Louisville, KY 40202

Email: islamiat.oladipupo@louisville.edu

\section{EDUCATION}

\section{5 - 2018 Ph.D. Public Health (Epidemiology)}

University of Louisville, Louisville, Kentucky

Chair: Kira C. Taylor, Ph.D., Associate Professor of

Epidemiology

Dissertation: Association Between Cigarette Smoking and

Ovarian Reserve Among Women Seeking Fertility

Treatment with Effect Modification by Race and NAT2

Genotype.

\section{2 - 2014 MPH Public Health (Epidemiology)}

University of Louisville, Louisville, Kentucky.

Advisor: Richard N. Baumgartner, Ph.D., Professor of

Epidemiology

Thesis: Assessing predictors of smoking cessation among participant in the Cooper Clayton smoking cessation program in Louisville, KY.

\section{$1999-2006$ MBBS Medicine and Surgery \\ Olabisi Onabanjo University, Ogun State, Nigeria.}




\section{RESEARCH EXPERIENCE}

2016 - Present Graduate Research Assistant, Louisville Tobacco Smoke Exposure, Genetic Susceptibility, \& Infertility Study, University of Louisville, Louisville, KY.

Description: Clinic-based research evaluating the interaction between NAT2 acetylator status and exposure to tobacco smoke on ovarian reserve and in- vitro fertilization outcomes. Research supported by a $\$ 0.45$ million grant from the Eunice Kennedy Shriver National Institute of Child Health and Human Development (NICHD), through the National Institute of Health (NIH) R15 parent program.

Projects: Association Between Active Smoking and Ovarian Reserve Among Females Seeking Fertility Treatment, and Interaction With N- Acetyltransferase 2(NAT2) Genotype.

Prevalence and Validity of Self-Reported Smoking Among Women Seeking Fertility Care.

2013 - $2014 \quad$ Epidemiology Intern, Department of Community Health and Prevention,

Louisville Metro Department of Public Health and Wellness, Louisville, Kentucky.

Description: Evaluated the Cooper Clayton smoking cessation program, a comprehensive behavioral smoking cessation program started over 25 years ago that utilizes education, skills training and social support.

\section{TEACHING EXPERIENCE}

2018 - Present Teaching Assistant, Department of Health Management and System Sciences, University of Louisville, Louisville, KY.

PHMS 301- Introduction to the U.S. Healthcare Delivery Systems Description: A graduate level course that explores different aspects of health care delivery in the United States with emphasis on role health professionals working in different types of health service organizations. Grade all quizzes, homework, assignment, and exams.

PHPH 431- Contemporary Issues in Public Health 
Description: An Undergraduate level course with an average of 80 students per semester. Developed course work, quizzes, and in-class activities. Grade all quizzes, homework, assignment, and exams.

PHUN 101- Introduction to Public Health

Description: An Undergraduate level course with an average of 80 students per semester. Grade all quizzes, homework, assignment, and exams. Met with students to answer any questions and mentor them.

\section{CLINICAL EXPERIENCE}

$2010-2012$

Medical Officer, Department of Obstetrics and Gynecology, Harvey Road Health Center, Lagos Nigeria

Description: Provided standard clinical care to patients daily (approximately 30 patients per day), consulted in emergency, gynecology, ante -natal and post-natal clinics and performed procedures such as manual vacuum aspiration, episioraphy, instrumental deliveries, normal deliveries and repair of laceration.

2008 - 2010 Medical Officer, Department of Obstetrics and Gynecology, Lagos Island Maternity Hospital, Lagos, Nigeria Description: Managed and treated life-threatening cases and emergencies in obstetrics and gynecology. Provided reproductive health and HIV/AIDS counseling to patient and participated in Prevention of Maternal to Child Transmission of HIV /AIDS. Worked with the AIDS Prevention Initiative of Nigeria (APIN) in collection, analysis and reporting of data on HIV /AIDS patients.

2006- 2007 House Officer, General Hospital, Lagos, Nigeria.

Description: Managed several cases in pediatrics, medicine, surgery, obstetrics and Gynecology under the supervision of consultants and specialists (approximately 40 per day).

\section{HONORS AND AWARDS}

2018 School of Interdisciplinary and Graduate Studies Certificate of Excellence, University of Louisville

2018 School of Public Health and Information Science Conference Travel Award, University of Louisville

2017 Graduate School Council Conference Travel Award, University of Louisville

2017 School of Interdisciplinary and Graduate Studies Fellowship Award, University of Louisville 
2016 University Fellowship, University of Louisville, KY

2014 Delta Omega National Public Health Honorary Award

2006 Best Graduating Medical Student (out of 150), Olabisi Onabanjo University

2002 Ogun State Scholarship Award for Academic Excellence

2002 Federal Government Scholarship Award for Academic Excellence

\section{PEER-REVIEWED PRESENTATIONS}

1. Oladipupo I, Ali T, Bohler H, Pagidas K, Torres S, Mann M, Gentry A, Dondik Y, Chiang J, Taylor K. Prevalence and Validity of Self-Reported Smoking Among Women Seeking Fertility Care. [Poster Presentation]. American College of Epidemiology (Annals of Epidemiology- Manuscript number- AEP_2018_288) Annual Meeting, September 2018.

2. Ali T, Oladipupo I, Bohler H, Pagidas K, Hein D, Mann M, Gentry A, Dondik Y, Chiang J, Taylor K. Childhood Secondhand Tobacco Smoke Exposure and Ovarian Reserve Among Females Seeking Fertility Care, and Interaction with NAcetyltransferase 2(NAT2) Genotype [Poster Presentation]. American College of Epidemiology (Annals of Epidemiology) Annual Meeting,2018.

3. Oladipupo I, Ali T, Bohler H, Pagidas K, Hein D, Mann M, Gentry A, Dondik Y, Chiang J, Taylor K. Association between Smoking and Ovarian Reserve Among Females Seeking Fertility Treatment, and Interaction with N- Acetyltransferase 2(NAT2) Genotype [Oral Presentation]. Society for Pediatric and Perinatal Epidemiologic Research Annual Meeting, Baltimore, Maryland, June 2018.

\section{NON-PEER REVIEWED PRESENTATIONS}

1. Oladipupo I. Assessing the predictors of smoking cessation among participants of the Cooper Clayton Smoking Cessation Program in Louisville, KY. Louisville Metro Department of Public Health and Wellness, Louisville, Kentucky April 2014.

2. Oladipupo I, Hickey C, Horlander L. Jones K, Smart A. Availability, Price, and Quality of Fresh Fruits and Vegetables: A Comparison of Select Stores in Louisville, Kentucky. School of Public Health and Information Sciences, University of Louisville, Louisville, Kentucky, April 2013.

3. Hickey C, Oladipupo I, Horlander L, Jones K, Smart A. Impact of Lead abatement policy in Kentucky on the incidence of Lead related illness in children of West Louisville, Kentucky. School of Public Health and Information Sciences, University of Louisville, Louisville, Kentucky, February 2013. 
4. Oladipupo I. Assessment of the land use types in relation to risk of pollution of three public parks in Jefferson county, Kentucky. Department of Geography, University of Louisville, Louisville, Kentucky, December 2013.

5. Oladipupo I. Community Assessment of Clark County, Kentucky. School of Public Health and Information Sciences, University of Louisville, Louisville, Kentucky, December 2012.

\section{MANUSCRIPTS IN PROGRESS}

1. Oladipupo I, Baumgartner KB, Hein DW, Wallis A, Gunaratnam B, and Taylor KC. Association Between Cigarette Smoking and Ovarian Reserve Among Women Seeking Fertility Treatment.

2. Oladipupo I, Baumgartner KB, Hein DW, Wallis A, Gunaratnam B J, and Taylor KC. Interaction Between NAT2 Acetylator Status and Active Exposure to Tobacco Smoke on Ovarian Reserve Among Women Seeking Fertility Care.

3. Oladipupo I, Baumgartner KB, Hein DW, Wallis A, Gunaratnam B, and Taylor KC. Racial Disparity in the Association of Cigarette Smoking with Ovarian Reserve Among Women Seeking Fertility Treatment.

\section{PROFESSIONAL AFFILIATIONS}

American College of Epidemiology (ACE) - Current Journal: Annals of Epidemiology

Society for Epidemiologic Research (SER) - Current Journals: American Journal of Epidemiology, Epidemiologic Reviews

Society for Pediatric and Perinatal Epidemiologic Research (SPER) - Current Journal: Pediatric and Perinatal Epidemiology

American Public Health Association (APHA) - Current

Kentucky Pubic Health association (KPHA) - Current

Nigerian Medical Association (NMA) - Current

Medical Guild Lagos - Past

\section{PROFESSIONAL WORKSHOPS}

- Principles of Data Management in Clinical and Epidemiologic Research, American College of Epidemiology (ACE), Annual Meeting Workshop, September 2018.

- Advanced Statistical Method workshop, Society for Pediatric and Perinatal Epidemiologic Research (SPER), Annual Meeting Workshop, June 2017. 